\title{
Bootstrap union tests for unit roots in the presence of nonstationary volatility
}

Citation for published version (APA):

Smeekes, S., \& Taylor, A. M. R. (2010). Bootstrap union tests for unit roots in the presence of nonstationary volatility. METEOR, Maastricht University School of Business and Economics. METEOR Research Memorandum No. 015 https://doi.org/10.26481/umamet.2010015

Document status and date:

Published: 01/01/2010

DOI:

10.26481/umamet.2010015

Document Version:

Publisher's PDF, also known as Version of record

\section{Please check the document version of this publication:}

- A submitted manuscript is the version of the article upon submission and before peer-review. There can be important differences between the submitted version and the official published version of record.

People interested in the research are advised to contact the author for the final version of the publication, or visit the DOI to the publisher's website.

- The final author version and the galley proof are versions of the publication after peer review.

- The final published version features the final layout of the paper including the volume, issue and page numbers.

Link to publication

\footnotetext{
General rights rights.

- You may freely distribute the URL identifying the publication in the public portal. please follow below link for the End User Agreement:

www.umlib.nl/taverne-license

Take down policy

If you believe that this document breaches copyright please contact us at:

repository@maastrichtuniversity.nl

providing details and we will investigate your claim.
}

Copyright and moral rights for the publications made accessible in the public portal are retained by the authors and/or other copyright owners and it is a condition of accessing publications that users recognise and abide by the legal requirements associated with these

- Users may download and print one copy of any publication from the public portal for the purpose of private study or research.

- You may not further distribute the material or use it for any profit-making activity or commercial gain

If the publication is distributed under the terms of Article $25 \mathrm{fa}$ of the Dutch Copyright Act, indicated by the "Taverne" license above, 
Stephan Smeekes, A. M. Robert Taylor

Bootstrap Union Tests for Unit Roots in the Presence of Nonstationary Volatility

$\mathrm{RM} / 10 / 015$

\section{METEOR}

Maastricht University School of Business and Economics Maastricht Research School of Economics

of Technology and Organization

\section{PO. Box 616}

NL - 6200 MD Maastricht

The Netherlands 


\title{
Bootstrap Union Tests for Unit Roots in the Presence of Nonstationary Volatility*
}

\author{
Stephan Smeekes \\ Department of Quantitative Economics \\ Maastricht University
}

\author{
A. M. Robert Taylor \\ School of Economics \\ University of Nottingham
}

February 2010

\begin{abstract}
We provide a joint treatment of three major issues that surround testing for a unit root in practice: uncertainty as to whether or not a linear deterministic trend is present in the data, uncertainty as to whether the initial condition of the process is (asymptotically) negligible or not, and the possible presence of nonstationary volatility in the data. Harvey, Leybourne and Taylor (2010, Journal of Econometrics, forthcoming) propose decision rules based on a four-way union of rejections of QD and OLS detrended tests, both with and without allowing for a linear trend, to deal with the first two problems. However, in the presence of nonstationary volatility these test statistics have limit distributions which depend on the form of the volatility process, making tests based on the standard asymptotic critical values invalid. We construct bootstrap versions of the four-way union of rejections test, which, by employing the wild bootstrap, are shown to be asymptotically valid in the presence of nonstationary volatility. These bootstrap union tests therefore allow for a joint treatment of all three of the aforementioned problems.
\end{abstract}

Keywords: Unit root; local trend; initial condition; asymptotic power; union of rejections decision rule; nonstationary volatility; wild bootstrap.

JEL Classification: C22.

\section{Introduction}

It is well known that the performance of unit root tests depends on a number of factors not observable by the practitioner applying the tests. Two such factors that have a profound impact on the power of these unit root tests, and in particular the popular (augmented) Dickey-Fuller $[\mathrm{DF}]$ tests, are that of deterministic trends and the initial condition.

\footnotetext{
${ }^{*}$ Correspondence to: Robert Taylor, School of Economics, University of Nottingham, Nottingham, NG7 2RD, U.K. E-mail: robert.taylor@nottingham.ac.uk
} 
In many economic applications it is important to allow for the presence of a linear trend. If a linear trend is present in the data, but not accounted for in the test, power will decrease dramatically, such that even for a fairly small trend, the unit root tests will never reject, even asymptotically. On the other hand, if a trend is absent from the data, but is accounted for in computing the test, then power also drops relative to the test which does not allow for a trend. To deal with this issue, Harvey, Leybourne, and Taylor (2009a) [HLT] construct a new test that is formed as a union of rejections of unit root tests with and without deterministic trend. They show that this union test can maintain high power irrespective of the true value of the trend. Moreover, by adjusting the critical values used to determine the individual rejections, the union test also maintains correct asymptotic size.

Similarly, the initial condition (defined as the deviation of the initial observation from the deterministic components) is also known to have a major impact on the power of unit root tests. As investigated by Müller and Elliott (2003), among others, the DF test with OLS detrending, denoted here as DF-OLS, suffers from low power relative to the DF test with quasidifferenced (QD) or GLS detrending, denoted as DF-QD, if the initial condition is small; while the opposite occurs if the initial condition is large. Moreover, as with the deterministic trend, the initial condition is not observed, thus leaving the practitioner without proper knowledge of which test to apply. HLT again propose a test based on a union of rejections, this time from the DF-OLS and DF-QD tests, to deal with this situation, and show that this test maintains good size and power across different values of the initial condition as well.

In practice these two factors cannot realistically be viewed in isolation of each other. Therefore Harvey, Leybourne, and Taylor (2010) [HLTb] extend the analysis of HLT, by considering the impact of both factors simultaneously. They propose a four-way union of rejections of DF-QD and DF-OLS tests, both with and without trend. A modified version that involves (inconsistent) pre-testing for both the initial condition and the linear trend is also proposed and shown to improve the power of the basic four-way union in certain cases.

A third factor which has an impact on the power of unit root tests, and even more so on the size of unit root tests, is the presence of nonstationary volatility in the innovations. Many macro-economic and financial time series to which unit root tests are applied, appear to display volatility shifts, and such nonstationary volatility effects can greatly influence the size of standard unit root tests, even asymptotically, as has been shown by Cavaliere and Taylor (2007, 2008), among others. A solution to this problem is analyzed by Cavaliere and Taylor $(2008,2009 a)$, who employ the wild bootstrap to capture the nonstationary volatility within the re-sampled data. They show that the wild bootstrap correctly reproduces the first-order limiting null distribution under nonstationary volatility, thereby allowing for the construction of asymptotically valid (pivotal) bootstrap tests. They also show that bootstrap tests perform well in finite samples.

However, just as with the treatment of HLT regarding uncertainty regarding the deter- 
ministic trend and the initial condition, it is difficult to argue, given empirical evidence, that these two factors can be analysed separately from nonstationary volatility. In fact, as will be shown in this paper, the union tests of HLTb suffer in just the same way as any "standard" asymptotic test will do in the presence of nonstationary volatility. Therefore, in this paper we propose a joint treatment dealing with uncertainty regarding the deterministic trend, the initial condition and nonstationary volatility. For this purpose we construct union tests using the wild bootstrap principle.

When extending the union tests of HLTb to a bootstrap setting, several complications arise, however. First, simply applying the bootstrap to the individual tests underlying the union does not control size. In this paper we show how to combine the individual tests in a union in a valid way using the bootstrap, an idea that is not dissimilar in spirit to the use of the bootstrap in multiple testing problems (cf. White, 2000; Romano and Wolf, 2005). Bayer and Hanck (2009) also use the bootstrap for combining tests for co-integration, although they use a different approach from the one we take here. Second, a way has to be found to deal with uncertainty regarding the deterministic trend, as incorporating a local trend into the bootstrap DGP presents some interesting problems. We will analyse the best way to do so in the paper.

As is well documented in the literature, see for example Chang and Park (2003), Paparoditis and Politis (2003) and Swensen (2003), the bootstrap also offers size improvements for unit root tests in small samples if serial correlation is present. This of course also holds for our union tests. However, as this has already been investigated extensively in this literature, we will not focus attention on this issue here.

The structure of the paper is as follows. In Section 2 the bootstrap union tests are introduced under the assumption of homoskedasticity, allowing to focus initially on the complications that arise from introducing the bootstrap in this setting. The bootstrap union tests in the presence of nonstationary volatility are subsequently investigated in Section 3. Section 4 offers some conclusions. All proofs are contained in the Appendix.

A word on notation. $\lfloor x\rfloor$ is the largest integer smaller than or equal to $x$. ' $x:=y$ ' (' $x=: y$ ') indicates that $x$ is defined by $y$ ( $y$ is defined by $x$ ). Convergence in distribution (probability) is denoted by $\stackrel{d}{\rightarrow}(\stackrel{p}{\rightarrow})$. As is usual in the literature, bootstrap quantities (conditional on the original sample) are indicated by appending a superscript $*$ to the standard notation. Convergence in distribution (probability) of bootstrap statistics is denoted $\stackrel{d^{*}}{\longrightarrow}\left(\stackrel{p^{*}}{\longrightarrow}\right)$, where this convergence is taken to take place in probability. $W(r)$ denotes a univariate standard Brownian motion. Finally, $\mathscr{D}=D[0,1]$ denotes the space of right continuous with left limit (càdlàg) processes. 


\section{Bootstrap Union Tests for Unit Toots in Homoskedastic Models}

In this section we introduce bootstrap union tests under the assumption of homoskedasticity. This allows us to focus on the complications arising from the bootstrap, while being able to work with asymptotically pivotal statistics. First, the model is introduced. Next, the DFQD and DF-OLS unit root tests and their bootstrap counterparts are discussed. Finally, a bootstrap version of the union is derived and the properties of the bootstrap union test are examined.

\subsection{The Model}

We consider the following data generating process (DGP).

$$
\begin{aligned}
& y_{t}=x_{t}+\mu+\beta_{T} t, \quad t=0,1, \ldots, T, \\
& x_{t}=\rho_{T} x_{t-1}+u_{t}, \quad t=1, \ldots, T \\
& u_{t}=\sum_{j=0}^{\infty} \psi_{j} \varepsilon_{t-j}=: \psi(L) \varepsilon_{t}, \quad\left(\psi_{0}=1\right)
\end{aligned}
$$

where $\rho_{T}:=1-c / T$. We wish to test whether or not $y_{t}$ contains a unit root; that is, our interest focuses on testing $H_{0}: c=0$ against $H_{1}: c>0$.

The stochastic process $\psi(L) \varepsilon_{t}$ is assumed to satisfy the following (standard) linear process condition.

\section{Assumption 1.}

(i) Let $\varepsilon_{t}$ be i.i.d. with $\mathrm{E} \varepsilon_{t}=0, \mathrm{E} \varepsilon_{t}^{2}=\sigma^{2}$ and $\mathrm{E} \varepsilon_{t}^{4}<\infty$.

(ii) $\psi(z) \neq 0$ for all $|z| \leq 1$, and $\sum_{j=0}^{\infty} j\left|\psi_{j}\right|<\infty$. Also define $\omega_{u}^{2}:=\lim _{T \rightarrow \infty} T^{-1} \mathrm{E}\left(\sum_{t=1}^{T} u_{t}\right)^{2}=$ $\sigma^{2} \psi(1)^{2}$.

Assumption 1(i) will be relaxed subsequently when we allow for nonstationary volatility. The next assumption specifies the behaviour of the coefficient on the linear trend term in (1), providing an appropriate Pitman (local) drift for our subsequent asymptotic analyses. This assumption coincides with that employed in HLT and HLTb.

Assumption 2. The trend coefficient $\beta_{T}$ in (1) satisfies $\beta_{T}:=T^{-1 / 2} \omega_{u} \kappa$.

As argued in HLT, it is appropriate to consider a local trend model in order that the subsequent asymptotic analysis reflects the uncertainty that exists in finite samples over whether a linear trend is present in the data or not. Finally, for the second source of uncertainty, the initial condition, we again follow HLT and HLTb and assume the following. 
Assumption 3. The initial condition, $x_{0}$, is generated by $x_{0}=\xi$, where $\xi:=\alpha \sqrt{\omega_{u}^{2}\left(1-\rho_{T}\right)^{-1}}$, for $\rho_{T}=1-c / T, c>0$, and for $c=0$ we set $\xi=0$ without loss of generality.

In Assumption 3, $\alpha$ controls the magnitude of $x_{0}$ relative to the magnitude of the standard deviation of a stationary $A R(1)$ process with parameter $\rho_{T}$ and innovation long-run variance $\omega_{u}^{2}$. The form given for $\xi$ is also consistent with the analysis of Müller and Elliott (2003) and Elliott and Müller (2006).

\subsection{Unit Root Tests and their Bootstrap Analogues in Homoskedastic Models}

As in HLT and HLTb, we consider the OLS- and QD-detrended DF unit root tests. Both tests involve an initial step of detrending to obtain the detrended series $\hat{x}_{t, \gamma}^{\delta}$ as

$$
\hat{x}_{t, \gamma}^{\delta}:=y_{t}-\hat{\theta}_{\gamma}^{\prime} z_{t}^{\delta}
$$

where

$$
\hat{\theta}_{\gamma}:=\left(\sum_{t=0}^{T} z_{\bar{c}, \gamma, t}^{\delta} z_{\bar{c}, \gamma, t}^{\delta \prime}\right)^{-1}\left(\sum_{t=0}^{T} z_{\bar{c}, \gamma, t}^{\delta} y_{\bar{c}, \gamma, t}\right) .
$$

Here $\gamma=Q D$ and $\gamma=O L S$ for QD and OLS detrending, respectively; hence,

$$
z_{\bar{c}, \gamma, t}^{\delta}:=\left\{\begin{array}{cl}
z_{t}^{\delta}-\left(1-\bar{c} T^{-1}\right) z_{t-1}^{\delta} & \text { if } \gamma=Q D, \\
z_{t}^{\delta} & \text { if } \gamma=O L S,
\end{array} \quad t \geq 1,\right.
$$

while $z_{\bar{c}, \gamma, 0}^{\delta}:=z_{0}^{\delta}$. Furthermore, $\delta=\mu, \tau$, where $z_{t}^{\mu}=1$ if just an intercept is included in the regression and $z_{t}^{\tau}=(1, t)^{\prime}$ if both an intercept and a linear trend are included. For QD detrending we take $\bar{c}=7$ and $\bar{c}=13.5$ if $\delta=\mu$ and $\delta=\tau$ respectively, as recommended by Elliott, Rothenberg, and Stock (1996).

The DF t-statistic $D F-\gamma^{\delta}$ is then the t-statistic of $\lambda$ in the augmented DF regression

$$
\Delta \hat{x}_{t, \gamma}^{\delta}=\lambda \hat{x}_{t-1, \gamma}^{\delta}+\sum_{j=1}^{p} \phi_{p, j} \Delta \hat{x}_{t-j}^{\delta}+\varepsilon_{p, t}, \quad t=p+1, \ldots, T .
$$

Furthermore, let $v_{t-1, p, \gamma}^{\delta}:=\left(\Delta \hat{x}_{t-1, \gamma}^{\delta}, \ldots, \Delta \hat{x}_{t-p, \gamma}^{\delta}\right)^{\prime}, \Delta \hat{X}_{\gamma}^{\delta}:=\left(\Delta \hat{x}_{p+1, \gamma}^{\delta}, \ldots, \Delta \hat{x}_{T, \gamma}^{\delta}\right)^{\prime}, \hat{X}_{-1, \gamma}^{\delta}:=$ $\left(\hat{x}_{p, \gamma}^{\delta}, \ldots, \hat{x}_{T-1, \gamma}^{\delta}\right)^{\prime}, V_{p, \gamma}^{\delta}:=\left(v_{p, p, \gamma}^{\delta}, \ldots, v_{T-1, p, \gamma}^{\delta}\right)^{\prime}$ and $M(Z):=I-Z\left(Z^{\prime} Z\right)^{-1} Z^{\prime}$. Then

$$
D F-\gamma^{\delta}:=\hat{\sigma}^{-1}\left(\hat{X}_{-1, \gamma}^{\delta \prime} M\left(V_{p, \gamma}^{\delta}\right) \hat{X}_{-1, \gamma}^{\delta}\right)^{-1 / 2} \hat{X}_{-1, \gamma}^{\delta \prime} M\left(V_{p, \gamma}^{\delta}\right) \Delta \hat{X}_{\gamma}^{\delta}
$$


where

$$
\hat{\sigma}^{2}:=T^{-1} \Delta \hat{X}_{\gamma}^{\delta \prime}\left[M\left(V_{p, \gamma}^{\delta}\right)-M\left(V_{p, \gamma}^{\delta}\right) \hat{X}_{-1, \gamma}^{\delta}\left\{\hat{X}_{-1, \gamma}^{\delta \prime} M\left(V_{p, \gamma}^{\delta}\right) \hat{X}_{-1, \gamma}^{\delta}\right\}^{-1} \hat{X}_{-1, \gamma}^{\delta \prime} M\left(V_{p, \gamma}^{\delta}\right)\right] \Delta \hat{X}_{\gamma}^{\delta} .
$$

We make the following (standard) assumption concerning the lag length, $p$ in (4).

Assumption 4. Let $p \rightarrow \infty$ and $p=o\left(T^{1 / 3}\right)$ as $T \rightarrow \infty$.

The limit distributions of the DF statistics under local alternatives are well known and documented in HLT and HLTb. For completeness we reproduce these results in the following Lemma.

Lemma 1. Let $y_{t}$ be generated according to (1) and let Assumptions 1, 2, 3 and 4 hold. Let $D F-\gamma^{\delta}$ be defined as in (5) with $\gamma=Q D, O L S$ and $\delta=\mu, \tau$. Then, as $T \rightarrow \infty$, we have that

$$
D F-\gamma^{\delta} \stackrel{d}{\rightarrow} \frac{K_{c, \gamma}^{\delta}(1, \kappa)^{2}-K_{c, \gamma}^{\delta}(0, \kappa)^{2}-1}{2\left(\int_{0}^{1} K_{c, \gamma}^{\delta}(r, \kappa)^{2} d r\right)^{1 / 2}}
$$

where

$$
\begin{aligned}
K_{c, Q D}^{\mu}(r, \kappa) & :=K_{c}(r)+r \kappa \\
K_{c, O L S}^{\mu}(r, \kappa) & :=K_{c}(r)-\int_{0}^{1} K_{c}(s) d s+\left(r-\frac{1}{2}\right) \kappa \\
K_{c, Q D}^{\tau}(r, \kappa) & :=K_{c}(r)-r\left(1+\bar{c}+\frac{1}{3} \bar{c}^{2}\right)^{-1}\left[(1+\bar{c}) K_{c}(1)+\bar{c}^{2} \int_{0}^{1} s K_{c}(s) d s\right], \\
K_{c, O L S}^{\tau}(r, \kappa) & :=K_{c}(r)-(4-6 r) \int_{0}^{1} K_{c}(s) d s-(12 r-6) \int_{0}^{1} s K_{c}(s) d s
\end{aligned}
$$

and

$$
K_{c}(r):=\left\{\begin{array}{cc}
W(r) & \text { if } c=0 \\
\alpha\left(e^{-r c}-1\right)(2 c)^{-1 / 2}+W_{c}(r) & \text { if } c>0
\end{array}\right.
$$

where $W_{c}(r):=\int_{0}^{r} e^{-(r-s) c} d W(s)$.

Remark 1: Observe that the limiting distributions of $D F-\gamma^{\tau}, \gamma=O L S, Q D$, do not depend on the local trend coefficient, $\kappa$, as would be expected given the exact invariance of these statistics to the trend parameter. In contrast, the limiting distributions of $D F-\gamma^{\mu}$, $\gamma=O L S, Q D$, depend on $\kappa$ both under the unit root null hypothesis, $c=0$, and under the alternative, $c>0$. The limiting distributions of all four statistics depend on $\alpha$, the initial condition magnitude, when $c>0$.

Various bootstrap versions of the above tests have been proposed; see Palm, Smeekes, and 
Urbain (2008) for a selective overview. Here we focus on DF tests using the sieve bootstrap (Park, 2002; Chang and Park, 2003; Smeekes, 2009) and the wild bootstrap (Cavaliere and Taylor, 2008, 2009a,b). It should be clear however that the same arguments hold for any other bootstrap method that delivers an asymptotically valid bootstrap test, such as the different forms of the block bootstrap (Paparoditis and Politis, 2003; Swensen, 2003; Parker, Paparoditis, and Politis, 2006). We start by describing our bootstrap algorithm.

\section{Algorithm 1.}

1. Calculate

$$
\hat{x}_{t, \tilde{\gamma}}^{\tau}=y_{t}-\hat{\theta}_{\tilde{\gamma}}^{\prime} z_{t}^{\tau}
$$

where $\hat{\theta}_{\tilde{\gamma}}$ is defined as in (3) with $\tilde{\gamma}=Q D, O L S$. It is not necessary that $\tilde{\gamma}$ is equal to $\gamma$, the detrending method used to obtain the test statistic.

2. Estimate an augmented DF regression of order $q$ for $\hat{x}_{t, \tilde{\gamma}}^{\tau}$ by OLS and calculate the residuals

$$
\hat{\varepsilon}_{q, t}^{\tau}:=\Delta \hat{x}_{t, \tilde{\gamma}}^{\tau}-\hat{\lambda} \hat{x}_{t-1, \tilde{\gamma}}^{\tau}-\sum_{j=1}^{q} \hat{\phi}_{q, j} \Delta \hat{x}_{t-j, \tilde{\gamma}}^{\tau}, \quad t=q+1, \ldots, T .
$$

3. Construct bootstrap errors $\varepsilon_{t}^{*}$ in one of the two following ways:

(i) (i.i.d. bootstrap) Resample with replacement from the recentered residuals $\left(\hat{\epsilon}_{q, t}^{\tau}-\overline{\hat{\varepsilon}}_{q, t}^{\tau}\right)$.

(ii) (wild bootsrap) Let $\varepsilon_{t}^{*}=\xi_{t}^{*} \hat{\varepsilon}_{q, t}^{\tau}$, where $\xi_{t}^{*}$ satisfies $\mathrm{E}^{*} \xi_{t}^{*}=0$ and $\mathrm{E}^{*} \xi_{t}^{* 2}=1$. $^{1}$

4. Build $u_{t}^{*}$ recursively as

$$
u_{t}^{*}=\sum_{j=1}^{q} \hat{\phi}_{q, j} u_{t-j}^{*}+\varepsilon_{t}^{*}
$$

using the estimated parameters $\hat{\phi}_{q, j}$ from Step 2, and build $x_{t}^{*}$ as

$$
x_{t}^{*}=x_{t-1}^{*}+u_{t}^{*}, \quad x_{0}^{*}=0 .
$$

Finally let

$$
y_{t}^{*}=x_{t}^{*}+\theta^{* \prime} z_{t}, \quad t=0,1, \ldots, T,
$$

where, either:

\footnotetext{
${ }^{1}$ In this paper we take $\xi_{t}^{*}$ to be standard normal. Other choices are also possible, although Cavaliere and Taylor (2008, Remark 6) mention that this has almost no impact on finite sample behaviour.
} 

A: $\theta^{*}=0$, or
$\mathrm{B}: \theta^{*}=\hat{\theta}_{\tilde{\gamma}}$.

5. Using the bootstrap sample $y_{t}^{*}$, apply the same method of detrending $\gamma$ as applied to the original sample to obtain the detrended bootstrap series

$$
\hat{x}_{t, \gamma}^{\delta *}:=y_{t}^{*}-\hat{\theta}_{\gamma}^{*} z_{t}^{\delta}
$$

where $\hat{\theta}_{\gamma}^{*}$ is defined analogously as in (3), but with the bootstrap data. Calculate the bootstrap augmented DF test statistic

$$
D F-\gamma_{\tilde{\gamma}}^{\delta *}:=\hat{\sigma}^{*-1}\left(\hat{X}_{-1, \gamma, \tilde{\gamma}}^{\delta * \prime} M\left(V_{p^{*}, \gamma, \tilde{\gamma}}^{\delta *}\right) \hat{X}_{-1, \gamma, \tilde{\gamma}}^{\delta *}\right)^{-1 / 2} \hat{X}_{-1, \gamma, \tilde{\gamma}}^{\delta * \prime} M\left(V_{p^{*}, \gamma, \tilde{\gamma}}^{\delta *}\right) \Delta \hat{X}_{\gamma, \tilde{\gamma}}^{\delta *}
$$

where all bootstrap sample quantities are defined analogously to their sample counterparts.

6. Repeat Steps 3 to $5 N$ times, obtaining bootstrap test statistics $D F-\gamma_{b}^{\delta *}$ for $b=$ $1, \ldots, N$, and select the bootstrap critical value $c_{\gamma, \tilde{\gamma}}^{\delta *}(\pi)$ as

$$
c_{\gamma, \tilde{\gamma}}^{\delta *}(\pi):=\max \left\{c: N^{-1} \sum_{b=1}^{N} I\left(D F-\gamma_{\tilde{\gamma}, b}^{\delta *}<c\right) \leq \pi\right\}
$$

or, equivalently, as the $\pi$-quantile of the ordered $D F-\gamma_{\tilde{\gamma}, b}^{\delta *}$ statistics. Reject the null of a unit root if $D F-\gamma^{\delta}$ is smaller than $c_{\gamma, \tilde{\gamma}}^{\delta *}(\pi)$, where $\pi$ is the nominal level of the test.

A crucial aspect of Algorithm 1 surrounds the choice of $\theta^{*}$. If $\delta=\tau$, then the bootstrap tests are invariant to the value of $\theta^{*}$, but just as with the asymptotic tests, they are not if $\delta=\mu$. We will denote by $D F-\gamma_{\tilde{\gamma}}^{\delta *}(A)$ and $D F-\gamma_{\tilde{\gamma}}^{\delta *}(B)$ the bootstrap DF statistics calculated using option A and B, respectively, in step 4 of Algorithm 1.

We also require the following assumptions on the lag lengths $p^{*}$ and $q$ used within the algorithm.

\section{Assumption 5.}

(i) Let $q \rightarrow \infty$ and $q=o\left((T / \ln T)^{1 / 3}\right)$ as $n \rightarrow \infty$.

(ii) Let $p^{*}$ satisfy Assumption 4 and let $p^{*} / q \rightarrow \kappa>1$ as $T \rightarrow \infty$, where $\kappa$ may be infinite.

Remark 2: The first part of Assumption 5 bounds $q$, while the second part essentially states that, for large $T, p^{*}$ should be at least as large as $q$. Often the lag length $p$ and $q$ will be identical (it is important to let $p^{*}$ differ though). However, we do not want to impose this, a priori, for two reasons. First, as can be seen from Assumptions 4 and 5(i), the assumptions 
on the rates are not the same. ${ }^{2}$ Second, and more importantly, the sieve regression might be based on a different specification than the test regression; in particular, if the test is based on demeaned data, then it is sensible to base the sieve regression on the detrended series in order to avoid any mis-specification. Indeed, we already advise to do just that in Algorithm 1. Later on, when we combine these tests in a union, this will become particularly important. In such cases it is not clear why the lag length with demeaning and detrending should be identical; hence, from the start, we allow for a different lag length in the two regressions.

We now detail the limit distributions of the bootstrap tests from Algorithm 1 in the following theorem.

Theorem 1. Let $y_{t}$ be generated according to (1) and let Assumptions 1, 2, 3, 4 and 5 hold. Then the bootstrap augmented DF t-statistics from Algorithm 1 satisfy, as $T \rightarrow \infty$,

(i) If either step 4.A or $4 . B$ is used

$$
D F-\gamma_{\tilde{\gamma}}^{\tau *}(A, B) \stackrel{d^{*}}{\longrightarrow} \frac{K_{0, \gamma}^{\tau}(1, \kappa)^{2}-K_{0, \gamma}^{\tau}(0, \kappa)^{2}-1}{2\left(\int_{0}^{1} K_{0, \gamma}^{\tau}(r, \kappa)^{2} d r\right)^{1 / 2}} \text { in probability } .
$$

(ii) If step 4.A is used

$$
D F-\gamma_{\tilde{\gamma}}^{\mu *}(A) \stackrel{d^{*}}{\rightarrow} \frac{K_{0, \gamma}^{\mu}(1,0)^{2}-K_{0, \gamma}^{\mu}(0,0)^{2}-1}{2\left(\int_{0}^{1} K_{0, \gamma}^{\mu}(r, 0)^{2} d r\right)^{1 / 2}} \text { in probability }
$$

(iii) If step $4 . B$ is used

$$
D F-\gamma_{\tilde{\gamma}}^{\mu *}(B) \stackrel{d^{*}}{\rightarrow} \frac{K_{c, \gamma, \tilde{\gamma}}^{\mu *}(1, \kappa)^{2}-K_{c, \gamma, \tilde{\gamma}}^{\mu *}(0, \kappa)^{2}-1}{2\left(\int_{0}^{1} K_{c, \gamma, \tilde{\gamma}}^{\mu *}(r, \kappa)^{2} d r\right)^{1 / 2}} \text { in probability }
$$

where

$$
\begin{aligned}
K_{c, Q D, \tilde{\gamma}}^{\mu *}(r, \kappa) & :=W(r)+r\left(\kappa+B_{c, \tilde{\gamma}}\right), \\
K_{c, O L S, \tilde{\gamma}}^{\mu *}(r, \kappa) & :=W(r)-\int_{0}^{1} W(s) d s+\left(r-\frac{1}{2}\right)\left(\kappa+B_{c, \tilde{\gamma}}\right),
\end{aligned}
$$

\footnotetext{
${ }^{2}$ The lag polynomial serves a different purpose in the augmented DF regression and the sieve regression; in the first case it should just eliminate the serial correlation, while in the second case it should not just eliminate it but also correctly replicate it (and, hence, consistently estimate it at a specified rate). Compare with the rates required for DF t-test and coefficient test in Chang and Park (2002); stronger assumptions on the lag length are needed for the coefficient test for the same reasons as above.
} 
and

$$
\begin{aligned}
B_{c, Q D} & :=\left(1+\bar{c}+\frac{1}{3} \bar{c}^{2}\right)^{-1}\left[(1+\bar{c}) K_{c}(1)+\bar{c}^{2} \int_{0}^{1} s K_{c}(s) d s\right] . \\
B_{c, O L S} & :=-6 \int_{0}^{1} K_{c}(s) d s+12 \int_{0}^{1} s K_{c}(s) d s,
\end{aligned}
$$

Remark 3: The result in part (a) of Theorem 1 establishes that the bootstrap $D F-\gamma_{\tilde{\gamma}}^{\tau *}$ statistics attains the same first-order limiting null distribution as the corresponding $D F-\gamma_{\tilde{\gamma}}^{\tau}$ statistics, regardless of whether scheme A or B is used in step 4 of Algorithm 1. This result is, of course, expected given the invariance properties of the statistics based on detrended data (Remark 1).

Remark 4: The results in parts (b) and (c) of Theorem 1 show that the choice between scheme $\mathrm{A}$ and $\mathrm{B}$ in step 4 makes a difference, even asymptotically, when considering the statistics based on demeaned data. Under scheme $\mathrm{A}$, where $\theta^{*}=0$ (i.e. where no estimated deterministic component is added to the bootstrap sample data), it is seen from part (b) that the bootstrap $D F-\gamma_{\tilde{\gamma}}^{\mu *}$ statistics attain the same first-order limiting null $(c=0)$ distributions as the corresponding $D F-\gamma_{\tilde{\gamma}}^{\mu}$ statistics when $\kappa=0$. Consequently, bootstrap tests based on $D F-\gamma_{\tilde{\gamma}}^{\mu *}(A)$ are asymptotically valid owing to the fact that the asymptotic tests based on $D F-\gamma_{\tilde{\gamma}}^{\mu}$ are based on the asymptotic critical value relevant for $\kappa=0$. Both the asymptotic tests and the bootstrap analogue tests under scheme A will therefore be conservative when $\kappa \neq 0$; see HLT. In contrast, under scheme B where the estimated deterministic component is added to the bootstrap data, we see from part (c) that the bootstrap tests based on the $D F-\gamma_{\tilde{\gamma}}^{\mu}(B)$ statistics are asymptotically invalid. This is caused by the fact that $\kappa$ cannot be estimated consistently and instead converges (when scaled) to the random limit, $B_{c, \tilde{\gamma}}$ whose form depends on whether QD or OLS demeaning is used and on the value of $c$ but $^{3}$ not on $\kappa$. As a consequence the bootstrap statistics do not replicate the limiting null distribution of the demeaned DF statistics.

While the estimate of $\kappa$ is not consistent we might still expect, however, that it will provide some information about the true value of $\kappa \cdot{ }^{4}$ This is especially so since in the bootstrap limit distribution $\kappa$ shows up in the same way as in the original limit distribution, while as noted above the term causing the invalidity, $B_{c, \gamma}$, does not depend on $\kappa$. For this reason we now investigate how large the influence of the term $B_{c, \gamma}$ is on the limit distributions in part (c) of Theorem 1. To this end we now graph the asymptotic critical values of $D F-Q D^{\mu}$ and $D F-O L S^{\mu}$ (these will, of course, coincide for $\kappa=0$ with those for their bootstrap analogues

\footnotetext{
${ }^{3}$ The dependence on $c$ will clearly have some impact on power (cf. Paparoditis and Politis, 2003, 2005). However, unreported simulations show that the influence of $c$ on the limiting distributions is very small, and therefore power is hardly compromised.

${ }^{4} \mathrm{~A}$ similar argument is given for the use of inconsistent pre-tests in HLTb.
} 
calculated under scheme A), together with the corresponding asymptotic bootstrap critical values for scheme $\mathrm{B}$ at a 0.05 nominal level for varying $\kappa$ in Figure 1 . The asymptotic critical values, as well as all other asymptotic results in the paper, were obtained by direct simulation of the relevant limiting representations, approximating the standard Brownian motion using i.i.d. $N(0,1)$ random variables, and with the integrals approximated by normalized sums of 1,000 steps. All simulations were performed in Gauss 8.0 using 50,000 Monte Carlo replications.

\section{InsERT Figure 1 ABOUT HERE}

It is seen from Figure 1 that the bootstrap critical values under scheme B clearly deviate from the asymptotic critical values, demonstrating the invalidity of the bootstrap. However, the deviation is not very large, and more importantly, the bootstrap limit distributions follow the same tendency to shift to the right as $\kappa$ increases as is seen in the limiting distributions of the demeaned DF statistics. Therefore, even though the bootstrap tests are invalid, they still mimic the distribution of the demeaned DF statistics to a reasonable degree. Hence, we will not discard these invalid tests at this stage, but still consider them as a potential option in forming union tests. Moreover, because in the union multiple tests are combined, the error made by the bootstrap under scheme B may be smoothed out.

Remark 5: It is straightforward to show that under a fixed trend, i.e. $\beta_{T}=\omega_{u} \kappa$, the bootstrap test $D F-\gamma_{\tilde{\gamma}}^{\mu *}(B)$ is asymptotically valid, contrary to the local trend case discussed above (a similar result is found in Parker et al., 2006). However, one could argue that the framework of a fixed trend is not the most appropriate to analyze trend uncertainty; a fixed trend can be picked up consistently by pre-tests, thus rendering union tests obsolete.

\subsection{Bootstrap Union Tests}

HLTb extend the work of HLT and propose a four-way union of rejections of $D F-Q D^{\mu}, D F-$ $Q D^{\tau}, D F-O L S^{\mu}$ and $D F-O L S^{\tau}$, thereby simultaneously dealing both with uncertainty about the trend and the initial condition. They also provide a scaling constant, $\tau_{\pi}$, with which to multiply the critical values of the four individual tests in order to control the asymptotic size of the union test. Let $c_{\gamma}^{\delta}(\pi)$ denote the asymptotic critical value of $D F-\gamma^{\delta}$ at nominal level $\pi$. Then we can denote the rejection rule by

$$
\text { Reject } \begin{aligned}
H_{0} \text { if }\{ & \left\{D F-Q D^{\mu}<\tau_{\pi} c_{Q D}^{\mu} \text { or } D F-Q D^{\tau}<\tau_{\pi} c_{Q D}^{\tau}\right. \\
& \text { or } \left.D F-O L S^{\mu}<\tau_{\pi} c_{O L S}^{\mu} \text { or } D F-O L S^{\tau}<\tau_{\pi} c_{O L S}^{\tau}\right\} .
\end{aligned}
$$


Alternatively, we may write as in Harvey, Leybourne, and Taylor (2009b)

$$
\begin{aligned}
\text { Reject } H_{0} \text { if } \min ( & D F-Q D^{\mu},\left(\frac{c_{Q D}^{\mu}(\pi)}{c_{Q D}^{\tau}(\pi)}\right) D F-Q D^{\tau},\left(\frac{c_{Q D}^{\mu}(\pi)}{c_{O L S}^{\mu}(\pi)}\right) D F-O L S^{\mu}, \\
& \left.\left(\frac{c_{Q D}^{\mu}(\pi)}{c_{O L S}^{\tau}(\pi)}\right) D F-O L S^{\tau}\right)<\tau_{\pi} c_{Q D}^{\mu},
\end{aligned}
$$

in which case we can denote the test statistic as

$$
\begin{aligned}
U R_{4}(\pi)=\min ( & D F-Q D^{\mu},\left(\frac{c_{Q D}^{\mu}(\pi)}{c_{Q D}^{\tau}(\pi)}\right) D F-Q D^{\tau}, \\
& \left.\left(\frac{c_{Q D}^{\mu}(\pi)}{c_{O L S}^{\mu}(\pi)}\right) D F-O L S^{\mu},\left(\frac{c_{Q D}^{\mu}(\pi)}{c_{O L S}^{\tau}(\pi)}\right) D F-O L S^{\tau}\right) .
\end{aligned}
$$

This last form proves particularly useful when setting up the bootstrap; the statistic is now in the form of a minimum over four numbers, which can easily be calculated in each bootstrap iteration. It should also be immediately clear that by bootstrapping this statistic, the bootstrap critical value will automatically incorporate the scaling constant $\tau_{\pi}$ needed to achieve the correct size. This form of bootstrap test statistic closely corresponds to the maximum based bootstrap statistics employed in White (2000) for the purpose of multiple testing.

Remark 6: In (12) the statistic has been scaled with respect to the distribution of $D F-Q D^{\mu}$ but this is obviously an arbitrary choice. In fact, we may write the statistic as

$$
\begin{aligned}
U R_{4}(\pi):=\min & \left(\left(\frac{x}{c_{Q D}^{\mu}(\pi)}\right) D F-Q D^{\mu},\left(\frac{x}{c_{Q D}^{\tau}(\pi)}\right) D F-Q D^{\tau}\right. \\
& \left.\left(\frac{x}{c_{O L S}^{\mu}(\pi)}\right) D F-O L S^{\mu},\left(\frac{x}{c_{O L S}^{\tau}(\pi)}\right) D F-O L S^{\tau}\right),
\end{aligned}
$$

for any $x<0$. In that case the criterion for rejection would be 'Reject $H_{0}$ if $U R_{4}(\pi)<\tau_{\pi} x$ '. It is clear then that (12) follows by setting $x=c_{Q D}^{\mu}(\pi)$.

Remark 7: HLTb also consider a modified union test, which in some situations has higher power than $U R_{4}$. It consists of performing "pre-tests" for a large initial condition and a deterministic trend. These tests are not true pre-tests, as the initial condition and local trend cannot be consistently estimated, but they do provide information on the magnitude of the initial condition and trend. If the test for a small initial condition is rejected, only the union of $D F-O L S^{\mu}$ and $D F-O L S^{\tau}$ is used. If the test for a trend is rejected, only the union of $D F-Q D^{\tau}$ and $D F-O L S^{\tau}$ is used. If both are rejected, only $D F-O L S^{\tau}$ is used. The exact procedure with scaling constants can be found in HLTb. We refer to this procedure as $U R_{4}^{m}$. 
We now proceed with the bootstrap version of HLTb's $U R_{4}(\pi)$ test. To that end, consider the following slightly modified union test statistic:

$$
\begin{aligned}
U R_{4}(\pi):=\min ( & D F-Q D^{\mu},\left(\frac{c_{Q D}^{\mu *}(\pi)}{c_{Q D}^{\tau *}(\pi)}\right) D F-Q D^{\tau}, \\
& \left.\left(\frac{c_{Q D}^{\mu *}(\pi)}{c_{O L S}^{\mu *}(\pi)}\right) D F-O L S^{\mu},\left(\frac{c_{Q D}^{\mu *}(\pi)}{c_{O L S}^{\tau *}(\pi)}\right) D F-O L S^{\tau}\right) .
\end{aligned}
$$

The difference with the previous definition of $U R_{4}$ is that the critical values $c_{\gamma}^{\delta}(\pi)$ have been replaced by the values $c_{\gamma}^{\delta *}(\pi)$, which may come from a bootstrap procedure (but do not have to be, as discussed in Remark 9 below). We now give the bootstrap algorithm.

Algorithm 2. Perform steps 1 to 4 of Algorithm 1 to obtain a bootstrap sample $y_{t}^{*}$.

5. Calculate $D F-Q D_{\tilde{\gamma}}^{\mu *}, D F-Q D_{\tilde{\gamma}}^{\tau *}, D F-O L S_{\tilde{\gamma}}^{\mu *}$ and $D F-O L S_{\tilde{\gamma}}^{\tau *}$ using the bootstrap sample $y_{t}^{*}$. Next calculate $U R_{4, \tilde{\gamma}}^{*}(\pi)$ as

$$
\begin{aligned}
U R_{4, \tilde{\gamma}}^{*}(\pi)=\min ( & D F-Q D_{\tilde{\gamma}}^{\mu *},\left(\frac{c_{Q D}^{\mu *}(\pi)}{c_{Q D}^{\tau *}(\pi)}\right) D F-Q D_{\tilde{\gamma}}^{\tau *}, \\
& \left.\left(\frac{c_{Q D}^{\mu *}(\pi)}{c_{O L S}^{\mu *}(\pi)}\right) D F-O L S_{\tilde{\gamma}}^{\mu *},\left(\frac{c_{Q D}^{\mu *}(\pi)}{c_{O L S}^{\tau *}(\pi)}\right) D F-O L S_{\tilde{\gamma}}^{\tau *}\right) .
\end{aligned}
$$

6. Repeat Steps 3 to $5 N$ times, obtaining bootstrap test statistics $U R_{4, \tilde{\gamma}, b}^{*}(\pi)$ for $b=$ $1, \ldots, N$, and select the bootstrap critical value as $c_{U R, \tilde{\gamma}}^{*}(\pi)=\max \left\{c: N^{-1} \sum_{b=1}^{N} I\left(U R_{4, \tilde{\gamma}, b}^{*}(\pi)<\right.\right.$ $c) \leq \pi\}$, or equivalently as the $\pi$-quantile of the ordered $U R_{4, \tilde{\gamma}, b}^{*}(\pi)$ statistics. Reject the null of a unit root if $U R_{4}(\pi)$ is smaller than $c_{U R, \tilde{\gamma}}^{*}(\pi)$.

We discern between $U R_{4, \tilde{\gamma}, A}^{*}$, constructed using $D F-\gamma_{\tilde{\gamma}}^{\mu *}(A)$ with $\theta^{*}=0$, and $U R_{4, \tilde{\gamma}, B}^{*}$, constructed using $D F-\gamma_{\tilde{\gamma}}^{\mu *}(B)$ with $\theta^{*}=\hat{\theta}_{\tilde{\gamma}}$.

Remark 8: It is important to construct just one bootstrap process from which to calculate all four statistics, and not construct four different bootstrap processes, in order to correctly replicate the distribution of the union; the original union statistic is also based on just one sample.

From now on we will ease notation by no longer indexing the $U R^{*}$ tests with respect to $\tilde{\gamma}$. As can be seen in Figure 1, it matters only very slightly whether $\tilde{\gamma}=Q D$ or $\tilde{\gamma}=O L S$ is used. This remains the same for the union tests we consider, therefore in the following we will always take $\tilde{\gamma}=O L S$ and simply refer to the bootstrap union tests as $U R_{4, A}^{*}$ or $U R_{4, B}^{*}$.

The limit distributions of the $U R_{4}$ and $U R_{4}^{*}$ statistics follow directly from the continuous mapping theorem and the limit distributions of the individual (bootstrap) DF statistics (cf. 
White, 2000, Proposition 2.2). It is therefore clear that $U R_{4, A}^{*}$ is asymptotically valid, having the same first-order limit null distribution as $U R_{4}$ when $\kappa=0$, while $U R_{4, B}^{*}$ is invalid because the underlying tests are invalid; cf. Remark 4.

Remark 9: There are two options for the choice of $c_{\gamma}^{\delta *}(\pi)$; one can take the asymptotic critical value, or one can take the bootstrap critical value resulting from bootstrapping the individual DF statistics. Asymptotically these are equivalent so from an asymptotic perspective the choice is irrelevant. In finite samples the bootstrap critical value may be preferable as it will usually be a better approximation of the true critical value than the asymptotic one. While it seems that using the bootstrap critical value might involve an additional bootstrap step to determine it, it can in fact be determined in the same bootstrap procedure as the calculation of $U R_{4}^{*}$ is done, as the individual DF statistics must be calculated anyway; hence no additional bootstrap iterations are necessary. One should further note that, if bootstrap critical values are used, $c_{\gamma}^{\mu *}$ should be based on the demeaned statistics from scheme A of step 4 the algorithm; that is, with $\theta^{*}=0$. If they are based on scheme $\mathrm{B}$, with $\theta^{*}=\hat{\theta}_{\tilde{\gamma}}$, too much weight will be given to the $D F-\gamma^{\mu}$ statistics for large $\kappa$, thus having a detrimental effect on power.

We will next analyse the asymptotic properties of the union test. We focus on the comparison of the asymptotic $U R_{4}$ test (and its bootstrap equivalent $U R_{4, A}^{*}$ ) with the bootstrap $U R_{4, B}^{*}$ test. We also add the $U R_{4}^{m}$ test of HLTb.

Figure 2 gives the asymptotic size for varying $\kappa$. The invalidity of the $U R_{4, B}^{*}$ test can be seen as the asymptotic size is above the nominal level of 0.05 for small $\kappa$. However, surprisingly the size does not rise above 0.06, making the size distortion rather modest. For large $\kappa$ the $U R_{4, B}^{*}$ test is not as conservative as the $U R_{4}$ and $U R_{4}^{m}$ tests, and its size appears to converge to the nominal level, which is as expected given Remark 5 . This might lead to the test having higher power for larger $\kappa$.

\section{INSERT Figure 2 ABOUT HERE}

Figures 3 to 8 show the asymptotic (uncorrected) power of the tests. For small $\kappa$ the power of $U R_{4, B}^{*}$ is very close to that of $U R_{4}$ and, hence, also to $U R_{4, A}^{*}$. From $\kappa=1$ on the power difference between $U R_{4, B}^{*}$ and $U R_{4}$ is noticeable, however the power of $U R_{4}^{m}$ is still higher for $\kappa=1 . \kappa$ has to increase to 2 that the power of $U R_{4, B}^{*}$ is higher than that of $U R_{4}^{m}$ (depending on the initial condition). For even larger $\kappa$, the power advantage of the $U R_{4, B}^{*}$ becomes greater, by virtue of the convergence of the size towards the nominal level. However, such a large $\kappa$ can effectively be considered as a fixed trend, and therefore this is arguably not the most relevant range to apply the union test to.

The impact of the magnitude of the initial condition on the power of the tests is somewhat more varied. In general the $U R_{4}$ and $U R_{4, B}$ tests tend to be relatively more powerful in 
comparison with the $U R_{4}^{m}$ test for a small initial condition (in absolute sense), while the opposite occurs for a large initial condition. Notice that the effect of the initial condition is not symmetrical around zero, as can clearly be seen from Figure 4 and 5 for $\kappa=0.25$ and $\kappa=0.5$. Moreover, there seems to be an interaction with $\kappa$; the intermediate values of $\kappa$ show different patterns across the values of the initial condition than the small and large values of $\kappa$.

\section{INSERT Figures 3-8 ABOUT HeRE}

To investigate if the finite sample performance of the individual bootstrap unit root tests discussed earlier carries over to the bootstrap union test, we perform a short Monte Carlo experiment for a small sample size. We only consider size here; we use DGP (1) with $c=0$ and $u_{t}=\varphi u_{t-1}+\varepsilon_{t}+\vartheta \varepsilon_{t-1}$, with $\varepsilon_{t} \sim N(0,1)$. We take $T=50$ and consider the $U R_{4, A}^{*}$, $U R_{4, B}^{*}$ and $U R_{4}^{m}$ tests. Lag length selection (also within the bootstrap) is done by MAIC (Ng and Perron, 2001) with a maximum lag length of $12(T / 100)^{1 / 4}$; as recommended by Perron and $\mathrm{Qu}(2007)$ we apply MAIC only to the OLS demeaned and detrended series. The sieve bootstrap regression is based on OLS detrended series (with lag length again selected by MAIC). We consider 5 combinations of $\varphi=\{-0.4,0,0.4\}$ and $\vartheta=\{-0.4,0,0.4\}$. We take 0.05 as nominal level of the tests. Results are based on 2000 simulations and 499 bootstrap replications. Simulations were programmed in Gauss 8.0.

\section{InSERT Figure 9 ABOUt HERE}

The results are given in Figure 9. The results for the model without serial correlation closely resemble the asymptotic results. If there is serial correlation, the bootstrap tests, as expected, have size closer to the nominal level than the asymptotic $U R_{4}^{m}$ test. Remarkably, the size correction not only occurs if the asymptotic test is oversized, but also if it undersized. These results are in line with the results for the individual bootstrap unit root tests (cf. Chang and Park, 2003; Smeekes, 2009). It is also noticeable that the $U R_{4, A}^{*}$ test in general has somewhat better size properties than $U R_{4, B}^{*}$.

Concluding, the bootstrap $U R_{4, B}^{*}$ test can indeed mimic the effect of the local trend, although at the cost of invalidity. As expected its power is higher than that of the conservative $U R_{4}$ test for larger values of $\kappa$. However the power difference only becomes noticeable for quite large values of $\kappa$, in particular in comparison to HLTb's $U R_{4}^{m}$ test. One can therefore raise the question of how much, at least asymptotically, the bootstrap $U R_{4, B}^{*}$ test, improves on the asymptotic tests, in particular as the price of invalidity has to be paid. In finite samples it may still have all the benefits over the asymptotic tests that all bootstrap tests have, but the same holds for the $U R_{4, A}^{*}$ test.

We will therefore now move on to a setting where the bootstrap test does have a large asymptotic advantage: the setting of nonstationary volatility. 


\section{Wild Bootstrap Union Tests with Nonstationary Volatility}

\subsection{Unit Root Testing in Models with Nonstationary Volatility}

Cavaliere and Taylor (2008) and Cavaliere and Taylor (2009b) consider testing for unit roots in settings where the volatility exhibits nonstationary behaviour. They show that standard unit root tests are asymptotically not correctly sized for such volatility processes. Therefore, they propose wild bootstrap tests that are robust to nonstationary volatility; not only are these tests asymptotically valid, they are also shown to perform very well in finite samples.

The issues of uncertainty about the presence of a trend and the initial condition cannot realistically be seen in isolation from the possible presence of nonstationary volatility. However, the asymptotic tests developed in HLTb can no longer be applied in the presence of nonstationary volatility, as the asymptotic unit root tests underlying the union are no longer correctly sized, even asymptotically. Moreover, it is impossible to find asymptotic scaling constants to control the asymptotic size of the union tests as these will depend on the form of the nonstationary volatility.

The bootstrap tests discussed in the previous section, however, do not suffer from these problems and retain their validity, provided the wild bootstrap variant is used. Hence, the bootstrap tests we consider here are robust to nonstationary volatility, trend uncertainty, and uncertainty about the initial condition. It is important to note that the scaling constants $c_{\gamma}^{\delta *}(\pi)$ must now chosen using the bootstrap critical values. The asymptotic critical values are no longer valid, and, moreover, the value of the critical values depends on the form of the volatility. It still holds that the bootstrap critical values should be based on the bootstrap samples generated with $\theta^{*}=0$.

In this paper we will follow the framework of Cavaliere and Taylor (2008), and use the following assumption concerning the form of nonstationary volatility allowed in the innovations.

\section{Assumption $1^{\prime}$.}

(i) Let $u_{t}=\psi(L) e_{t}$, where $e_{t}=\sigma_{t} \varepsilon_{t}$, and let $\psi(z)$ and $\varepsilon_{t}$ satisfy Assumption 1 (with $\left.\mathrm{E} \varepsilon_{t}^{2}=1\right)$.

(ii) The volatility term $\sigma_{t}$ satisfies $\sigma_{\lfloor T r\rfloor}=\omega(r)$ for all $r \in[0,1]$, where $\omega(\cdot) \in \mathscr{D}$ is nonstochastic and strictly positive. For $t<0, \sigma_{t} \leq \bar{\sigma}<\infty$.

This assumption allows for a wide variety of volatility processes, as the innovation variance is only required to be nonstochastic, bounded and to display a finite number of jumps. As discussed in detail by Cavaliere and Taylor (2008), this class includes variance with single abrupt break, multiple volatility shifts, polynomially trending volatility and smooth transition variance breaks. 
As in Cavaliere and Taylor (2008) we define the variance profile $\eta(r)$ as

$$
\eta(r):=\left(\int_{0}^{1} \omega(s)^{2} d s\right)^{-1}\left(\int_{0}^{r} \omega(s)^{2} d s\right) .
$$

Furthermore we define $\bar{\omega}^{2}:=\int_{0}^{1} \omega(s)^{2} d s$, which equals the limit of $T^{-1} \sum_{t=1}^{T} \sigma_{t}^{2}$ and may therefore be interpreted as the asymptotic average variance. Note that Assumptions 2 and 3 remain unchanged, although now $\omega_{u}^{2}=\bar{\omega}^{2} \psi(1)^{2}$.

We now state the limiting distributions of the $D F-\gamma^{\delta}$ statistics, the proof of which is a simple adaptation of the proof of Theorem 1 of Cavaliere and Taylor (2008).

Lemma 2. Let $y_{t}$ be generated according to (1) and let Assumptions 1', 2, 3 and 4 hold. Let $D F-\gamma^{\delta}$ be defined as in (5) with $\gamma=Q D, O L S$ and $\delta=\mu, \tau$. Then, as $T \rightarrow \infty$, we have that

$$
D F-\gamma^{\delta} \stackrel{d}{\rightarrow} \frac{K_{\eta, c, \gamma}^{\delta}(1, \kappa)^{2}-K_{\eta, c, \gamma}^{\delta}(0, \kappa)^{2}-1}{2\left(\int_{0}^{1} K_{\eta, c, \gamma}^{\delta}(r, \kappa)^{2} d r\right)^{1 / 2}},
$$

where

$$
\begin{aligned}
K_{\eta, c, Q D}^{\mu}(r, \kappa) & :=K_{\eta, c}(r)+r \kappa, \\
K_{\eta, c, O L S}^{\mu}(r, \kappa) & :=K_{\eta, c}(r)-\int_{0}^{1} K_{\eta, c}(s) d s+\left(r-\frac{1}{2}\right) \kappa, \\
K_{\eta, c, Q D}^{\tau}(r, \kappa) & :=K_{\eta, c}(r)-r\left(1+\bar{c}+\frac{1}{3} \bar{c}^{2}\right)^{-1}\left[(1+\bar{c}) K_{\eta, c}(1)+\bar{c}^{2} \int_{0}^{1} s K_{\eta, c}(s) d s\right], \\
K_{\eta, c, O L S}^{\tau}(r, \kappa) & :=K_{\eta, c}(r)-(4-6 r) \int_{0}^{1} K_{\eta, c}(s) d s-(12 r-6) \int_{0}^{1} s K_{\eta, c}(s) d s,
\end{aligned}
$$

and

$$
K_{\eta, c}(r):=\left\{\begin{array}{cc}
W_{\eta, 0}(r) & \text { if } c=0 \\
\alpha\left(e^{-r c}-1\right)(2 c)^{-1 / 2}+W_{\eta, c}(r) & \text { if } c>0
\end{array}\right.
$$

where $W_{\eta, c}(r):=\int_{0}^{r} e^{-(r-s) c} d W(\eta(s))$.

We next present the limiting distributions of the bootstrap DF test statistics. As noted previously, the bootstrap tests as described in the previous section remain valid, provided the wild bootstrap is used in Step 3 of Bootstrap Algorithm 1. In what follows we therefore make reference only to the wild bootstrap version of Algorithm 1.

Theorem 2. Let $y_{t}$ be generated according to (1) and let Assumptions 1', 2, 3, 4 and 5 hold. Let DF $-\gamma_{\tilde{\gamma}}^{\delta *}$ denote the bootstrap augmented DF t-statistics from Algorithm 1 with $\gamma, \tilde{\gamma}=Q D, O L S$ and $\delta=\mu, \tau$. Then, as $T \rightarrow \infty$, we have that 
(i) If either step 4.A or $4 . B$ is used

$$
D F-\gamma_{\tilde{\gamma}}^{\tau *}(A, B) \stackrel{d^{*}}{\longrightarrow} \frac{K_{\eta, 0, \gamma}^{\tau}(1, \kappa)^{2}-K_{\eta, 0, \gamma}^{\tau}(0, \kappa)^{2}-1}{2\left(\int_{0}^{1} K_{\eta, 0, \gamma}^{\tau}(r, \kappa)^{2} d r\right)^{1 / 2}} \quad \text { in probability } .
$$

(ii) If step 4.A is used

$$
D F-\gamma_{\tilde{\gamma}}^{\mu *}(A) \stackrel{d^{*}}{\longrightarrow} \frac{K_{\eta, 0, \gamma}^{\mu}(1,0)^{2}-K_{\eta, 0, \gamma}^{\mu}(0,0)^{2}-1}{2\left(\int_{0}^{1} K_{\eta, 0, \gamma}^{\mu}(r, 0)^{2} d r\right)^{1 / 2}} \quad \text { in probability. }
$$

(iii) If step 4.B is used

$$
D F-\gamma_{\tilde{\gamma}}^{\mu *}(B) \stackrel{d^{*}}{\longrightarrow} \frac{K_{\eta, c, \gamma, \tilde{\gamma}}^{\mu *}(1, \kappa)^{2}-K_{\eta, c, \gamma, \tilde{\gamma}}^{\mu *}(0, \kappa)^{2}-1}{2\left(\int_{0}^{1} K_{\eta, c, \gamma, \tilde{\gamma}}^{\mu *}(r, \kappa)^{2} d r\right)^{1 / 2}} \quad \text { in probability }
$$

where

$$
\begin{aligned}
K_{\eta, c, Q D, \tilde{\gamma}}^{\mu *}(r, \kappa) & :=W_{\eta, 0}(r)+r\left(\kappa+B_{\eta, c, \tilde{\gamma}}\right) \\
K_{\eta, c, O L S, \tilde{\gamma}}^{\mu *}(r, \kappa) & :=W_{\eta, 0}(r)-\int_{0}^{1} W_{\eta, 0}(s) d s+\left(r-\frac{1}{2}\right)\left(\kappa+B_{\eta, c, \tilde{\gamma}}\right),
\end{aligned}
$$

and

$$
\begin{aligned}
B_{\eta, c, Q D} & :=\left(1+\bar{c}+\frac{1}{3} \bar{c}^{2}\right)^{-1}\left[(1+\bar{c}) K_{\eta, c}(1)+\bar{c}^{2} \int_{0}^{1} s K_{\eta, c}(s) d s\right] . \\
B_{\eta, c, O L S} & :=-6 \int_{0}^{1} K_{\eta, c}(s) d s+12 \int_{0}^{1} s K_{\eta, c}(s) d s .
\end{aligned}
$$

Remark 10: The implications of the results in Theorem 2 are qualitatively similar to those from the results in Theorem 1 for the constant volatility case. Principally, the detrended wild bootstrap DF statistics attain the same first-order limit null distribution as the corresponding detrended DF statistics. This result has already been established in Cavaliere and Taylor (2008). For the bootstrap demeaned DF statistics, again the choice between schemes A and B in step 4 of Algorithm 1 is crucial. Asymptotically valid bootstrap tests are again obtained under scheme A, but not under scheme B. Notice that under scheme B, the additional random term in the limit distribution, $B_{\eta, c, \tilde{\gamma}}$ now also depends on the form of the nonstationary volatility.

Remark 11: As demonstrated in Cavaliere and Taylor (2008), usually one does not need Assumption 5 when applying the wild bootstrap, and it suffices to assume that $q \leq p^{*}$, where neither is required to increase with the sample size. This is also true in our setting 
for deriving the limit distributions of $D F-\gamma^{\tau *}$ and $D F-\gamma^{\mu *}(A)$, but it is not true for the limit distributions of $D F-\gamma^{\mu *}(B)$. For this test the nuisance parameters arising from the estimation of $\theta^{*}$ (i.e. $\omega_{u}$ ) imply that these must also be correctly reproduced within the bootstrap for them to cancel out in the limiting distribution.

Remark 12: Given the results in Cavaliere and Taylor (2009b), it would be possible to extend the framework to allow for a wider class of volatility processes, including nonstationary stochastic volatility and GARCH processes. Nothing would change in the set up of the tests, only the theory would become more involved. We do not consider this further here for expositional simplicity.

\subsection{Wild Bootstrap Union Tests}

The asymptotic and bootstrap distributions of the $U R_{4}^{*}$ tests follow directly from the continuous mapping theorem. Therefore, we expect the bootstrap union tests to be able to reproduce the impact of the volatility on the asymptotic distribution, unlike the asymptotic union tests. To investigate this, we simulate the asymptotic distributions of $U R_{4}, U R_{4, A}^{*}$ and $U R_{4, B}^{*}$ for a number of different models for the volatility. ${ }^{5}$ In particular, we consider the following settings that correspond to the models used in the small sample simulations in Cavaliere and Taylor (2008):

1. Single break in volatility: $\sigma_{t}^{2}=\sigma_{0}^{2}+\left(\sigma_{1}^{2}-\sigma_{0}^{2}\right) I(t>\lfloor\tau T\rfloor)$.

2. Double break in volatility: $\sigma_{t}^{2}=\sigma_{0}^{2}+\left(\sigma_{1}^{2}-\sigma_{0}^{2}\right) I(\lfloor\tau T\rfloor<t<\lfloor(1-\tau) T\rfloor)$.

3. Trending volatility: $\sigma_{t}=\sigma_{0}+\left(\sigma_{1}-\sigma_{0}\right) t$.

Figure 10 gives the results for size of these models. The asymptotic union test $U R_{4}$ is, as expected, not correctly sized, and in some situations it is quite severely oversized. The bootstrap tests behave in exactly the same way as in the i.i.d. case; $U R_{4, A}^{*}$ is conservative, its size decreases as $\kappa$ increases, while $U R_{4, B}^{*}$ is slightly oversized for small $\kappa$ but correctly sized when $\kappa$ increases. The fact that the behaviour of the bootstrap tests is the same over all combinations considered here is very encouraging, as it indicates that the bootstrap tests are highly robust to nonstationary volatility, and we may therefore generalize the conclusions drawn from the i.i.d. case.

\section{INSERT FIGURE 10 ABOUT HERE}

\footnotetext{
${ }^{5}$ We do not provide finite sample results here; given the asymptotic results it is clear that the bootstrap tests will outperform the asymptotic test. Simulation results (without serial correlation) in fact show that the bootstrap tests outperform the asymptotic $U R_{4}$ test even more so in small samples than asymptotically. For $T=50$ and $\kappa=0$, at a nominal level of 0.05 , the size of the asymptotic $U R_{4}$ test varies between 0.10 and 0.25 for the volatility models considered, while the size for the bootstrap tests is always between 0.04 and 0.07 . These results are available from the website www.personeel.unimaas.nl/s.smeekes/research.htm.
} 
We now turn to a consideration of the power properties of these tests. We also add the $D F-O L S^{* \tau}$ test to the graphs, for the same reason as HLTb do: this test is the only one of the four individual tests considered that does not have trivial power for any parameter combination. Moreover, since it is based on the wild bootstrap, it is also feasible and asymptotically valid. To be of practical value, therefore, the bootstrap union tests should provide power advantages over this test for at least a reasonable part of the parameter space. As the size of the asymptotic test $U R_{4}$ is often far from the nominal level, we size-correct the power at $\kappa=0$. Hence, the power is corrected for the nonstationary volatility, but not for the trend.

\section{InSERT Figures 11-22 ABOUt HeRE}

Power graphs are presented in Figures 11 to 22. Given the amount of space needed to display the graphs, we only report results for a subset of the models considered for size. This subset is representative of the remaining unreported cases. ${ }^{6}$ The union tests are seen to be substantially more powerful than the $D F-O L S^{\tau *}$ test for a significant subset of the parameter combinations, mostly those consisting of small $\kappa$ and small $\alpha$, while the power loss with respect to the $D F-O L S^{\tau *}$ test for the other combinations is relatively minor in most cases. Thus, the union tests are able to deal with the trend and initial condition uncertainty, just as in the homoskedastic case analysed by HLT and HLTb. Moreover, the bootstrap tests are also highly robust to the presence of nonstationary volatility in the innovations.

It is worth noting that the power properties of the tests can considerably differ in specific models. This can for example be seen for the trending volatility model, where the union test also offers (unexpected) power gains on the $D F-O L S^{* \tau}$ test for large $|\alpha|$. This can be explained by the fact that the nonstationary volatility also has a direct effect on the size-corrected local power function, a point also noted by Cavaliere and Taylor (2008, p. 8). Therefore, as is also clear from the shape of the power curves for the double break model, it should not be expected that the power curves, and hence also the relations between the tests, are exactly the same as in the i.i.d. case.

Comparing the two bootstrap union tests, we see that as in the homoskedastic case, the $U R_{4, B}^{*}$ test is somewhat more powerful than the $U R_{4, A}^{*}$ for $\kappa>0$, but the power difference only becomes substantial for large $\kappa$. It is therefore doubtful if this relatively minor power gain makes it worthwhile using a test that is invalid. ${ }^{7}$

Given the results presented here, the bootstrap union tests proposed in this paper would appear to constitute a valuable option if one needs to deal simultaneously with uncertainty regarding the trend, the initial condition and the presence of nonstationary volatility. Extant

\footnotetext{
${ }^{6}$ The full set of graphs is available from the authors upon request.

${ }^{7}$ Even though the distribution of $U R_{4, A}^{*}$ is only identical to the asymptotic distribution of the union test for $\kappa=0$, it is valid in the sense that it is a size $\pi$ test, whereas $U R_{4, B}^{*}$ clearly is not; cf. Remarks 4 and 10 .
} 
tests in the literature cannot perform satisfactorily in this situation, with the possible exception of the wild bootstrap $D F-O L S^{\tau *}$ test. However, as shown in this section, the bootstrap union tests have a clear power advantage over this test for those combinations of $\kappa$ and $\alpha$ that will indeed lead to uncertainty about their values, while for the other combinations of these parameters the power loss is quite modest.

\section{Conclusion}

In this paper we have developed bootstrap tests designed to be robust over uncertainty about the presence of a deterministic trend and uncertainty about the initial condition, thereby extending the union tests of HLT and HLTb to a bootstrap setting. Moreover, by employing the wild bootstrap, our proposed bootstrap tests were shown to be additionally robust to the presence of nonstationary volatility in the innovations.

We considered two bootstrap union tests, $U R_{4, A}^{*}$ and $U R_{4, B}^{*}$, the first is a valid (conservative) test, the second is an invalid test, although its size does not appear to deviate to any great degree from the nominal level and it is somewhat more powerful. In the setting of homoskedasticity the first test is asymptotically equivalent to the asymptotic $U R_{4}$ test of HLTb, while the second closely resembles it. Despite not offering any asymptotic improvements under homoskedasticity, simulation evidence demonstrated that the proposed bootstrap tests can still deliver an improvement in finite sample performance over the asymptotic union tests.

In cases where the volatility of the innovations is nonstationary, the asymptotic union tests of HLTb fail, just as regular asymptotic tests do. Here the wild bootstrap is used as in Cavaliere and Taylor $(2008,2009 b)$ for making our bootstrap union tests robust to nonstationary volatility. The power properties of the bootstrap union test in relation to the trend and initial condition remain similar to those which pertain in the homoskedastic case. Hence, in this setting the bootstrap union tests clearly provides clear advantages over the existing tests both asymptotically and in finite samples, as was demonstrated through simulation evidence.

\section{References}

Bayer, C. and C. Hanck (2009). Combining non-cointegration tests. METEOR Research Memorandum 09/012.

Cavaliere, G. and A. M. R. Taylor (2007). Testing for unit roots in time series models with non-stationary volatility. Journal of Econometrics 140, 919-947.

Cavaliere, G. and A. M. R. Taylor (2008). Bootstrap unit root tests for time series with nonstationary volatility. Econometric Theory 24, 43-71. 
Cavaliere, G. and A. M. R. Taylor (2009a). Bootstrap $M$ unit root tests. Econometric Reviews 28, 393-421.

Cavaliere, G. and A. M. R. Taylor (2009b). Heteroskedastic time series with a unit root. Econometric Theory 25, 1228-1276.

Chang, Y. and J. Y. Park (2002). On the asymptotics of ADF tests for unit roots. Econometric Reviews 21, 431-447.

Chang, Y. and J. Y. Park (2003). A sieve bootstrap for the test of a unit root. Journal of Time Series Analysis 24, 379-400.

Elliott, G. and U. K. Müller (2006). Minimizing the impact of the initial condition on testing for unit roots. Journal of Econometrics 135, 285-310.

Elliott, G., T. J. Rothenberg, and J. H. Stock (1996). Efficient tests for an autoregressive unit root. Econometrica 64, 813-836.

Harvey, D. I., S. J. Leybourne, and A. M. R. Taylor (2009a). Unit root testing in practice: dealing with uncertainty over the trend and initial condition. Econometric Theory 25, $587-636$.

Harvey, D. I., S. J. Leybourne, and A. M. R. Taylor (2009b). Unit root testing in practice: dealing with uncertainty over the trend and initial condition - rejoinder. Econometric Theory 25, 658-667.

Harvey, D. I., S. J. Leybourne, and A. M. R. Taylor (2010). Testing for unit roots in the presence of uncertainty over both the trend and initial condition. Journal of Econometrics, forthcoming.

Müller, U. K. and G. Elliott (2003). Tests for unit roots and the initial condition. Econometrica $71,1269-1286$.

Ng, S. and P. Perron (2001). Lag length selection and the construction of unit root tests with good size and power. Econometrica 69, 1519-1554.

Palm, F. C., S. Smeekes, and J.-P. Urbain (2008). Bootstrap unit root tests: comparison and extensions. Journal of Time Series Analysis 29, 371-401.

Paparoditis, E. and D. N. Politis (2003). Residual-based block bootstrap for unit root testing. Econometrica 71, 813-855.

Paparoditis, E. and D. N. Politis (2005). Bootstrapping unit root tests for autoregressive time series. Journal of the American Statistical Association 100, 545-553. 
Park, J. Y. (2002). An invariance principle for sieve bootstrap in time series. Econometric Theory 18, 469-490.

Parker, C., E. Paparoditis, and D. N. Politis (2006). Unit root testing via the stationary bootstrap. Journal of Econometrics 133, 601-638.

Perron, P. and Z. Qu (2007). A simple modification to improve the finite sample properties of $\mathrm{Ng}$ and Perron's unit root tests. Economics Letters 94, 12-19.

Phillips, P. C. B. and V. Solo (1992). Asymptotics for linear processes. Annals of Statistics 20, 971-1001.

Romano, J. P. and M. Wolf (2005). Stepwise multiple testing as formalized data snooping. Econometrica 73, 1237-1282.

Smeekes, S. (2009). Detrending bootstrap unit root tests. METEOR Research Memorandum 09/056, Maastricht University.

Stock, J. H. (1994). Unit roots, structural breaks and trends. In R. F. Engle and D. L. McFadden (Eds.), Handbook of Econometrics, Volume 4, Chapter 46, pp. 2739-2841. Amsterdam: North Holland Publishing.

Swensen, A. R. (2003). Bootstrapping unit root tests for integrated processes. Journal of Time Series Analysis 24, 99-126.

White, H. (2000). A reality check for data snooping. Econometrica 68, 1097-1126.

\section{A Appendix}

Proof of Theorem 1. We focus here on the proof for the sieve bootstrap test. The proof for the wild bootstrap follows similarly, and is a special case of the results derived in Section 3 .

It follows from results in, among others, Park (2002), that under Assumptions 1 and 5,

$$
T^{-1 / 2} \sum_{t=1}^{\lfloor T r\rfloor} u_{t}^{*} \stackrel{d^{*}}{\longrightarrow} \omega_{u} W(r) \quad \text { in probability. }
$$

The results in (i) and (ii) then follow as in Smeekes (2009). We now focus on (iii). Let $\theta^{*}:=\left(\mu^{*}, \beta^{*}\right)^{\prime}$ and note that for OLS detrending

$$
\hat{x}_{t, O L S}^{\mu *}=y_{t}^{*}-T^{-1} \sum_{t=1}^{T} y_{t}^{*}=x_{t}^{*}-T^{-1} \sum_{t=1}^{T} x_{t}^{*}+\beta^{*} t-\frac{1}{2}(T+1) \beta^{*} .
$$


Then

$$
\begin{aligned}
T^{-1 / 2} \hat{x}_{\lfloor T r\rfloor, O L S}^{\mu *}= & T^{-1 / 2}\left(x_{\lfloor T r\rfloor}^{*}-T^{-1} \sum_{t=1}^{T} x_{t}^{*}\right)+T^{1 / 2}\left(\frac{\lfloor T r\rfloor}{T}-\frac{1}{2}\right) \beta_{T} \\
& +T^{1 / 2}\left(\frac{\lfloor T r\rfloor}{T}-\frac{1}{2}\right)\left(\beta^{*}-\beta_{T}\right)+o_{p}^{*}(1) \\
& \stackrel{d^{*}}{\longrightarrow} \omega_{u}\left[W(r)-\int_{0}^{1} W(r) d r+\left(r-\frac{1}{2}\right)\left(\kappa+B_{c, \tilde{\gamma}}\right)\right] \quad \text { in probability, }
\end{aligned}
$$

as

$$
T^{1 / 2}\left(\beta^{*}-\beta_{T}\right)=T^{1 / 2}\left(\hat{\beta}_{\tilde{\gamma}}-\beta_{T}\right) \stackrel{d}{\rightarrow} B_{c, \tilde{\gamma}}
$$

which can easily be derived from standard results (cf. Stock, 1994; Elliott et al., 1996). For QD detrending we can derive in a similar way that

$$
\begin{aligned}
T^{-1 / 2} \hat{x}_{\lfloor T r\rfloor, Q D}^{\mu *} & =T^{-1 / 2} x_{\lfloor T r\rfloor}^{*}+T^{1 / 2} \frac{\lfloor T r\rfloor}{T} \beta+T^{1 / 2} \frac{\lfloor T r\rfloor}{T}\left(\beta^{*}-\beta\right)+o_{p}^{*}(1) \\
& \stackrel{d^{*}}{\longrightarrow} W(r)+r\left(\kappa+B_{c, \tilde{\gamma}}\right) \quad \text { in probability, }
\end{aligned}
$$

Result (iii) then follows in the same way as (i) and (ii).

Proof of Lemma 2. For $c=0$ it follows from Cavaliere and Taylor (2007, Theorem 1 ) that

$$
T^{-1 / 2} x_{\lfloor T r\rfloor} \stackrel{d}{\rightarrow} \bar{\omega} \psi(1) W_{\eta, 0}(r),
$$

while for $c>0$ we can write

$$
\begin{aligned}
T^{-1 / 2} x_{\lfloor T r\rfloor} & =T^{-1 / 2} \sum_{t=1}^{\lfloor T r\rfloor} \Delta x_{t}+T^{-1 / 2} x_{0} \\
& =T^{-1 / 2} \sum_{t=1}^{\lfloor T r\rfloor} \Delta x_{t}+T^{-1 / 2} \alpha \sqrt{\omega_{u}^{2}\left(1-\rho_{T}\right)^{-1}} \\
& \stackrel{d}{\rightarrow} \bar{\omega} \psi(1)\left[W_{\eta, c}(r)+\alpha\left(e^{-r c}-1\right)(2 c)^{-1 / 2}\right] .
\end{aligned}
$$

Furthermore, by Lemma 2 in Cavaliere and Taylor (2008) we have that $\hat{\sigma}^{2} \stackrel{p}{\rightarrow} \bar{\omega}(s)^{2}$. Cavaliere and Taylor (2007, Theorem 1) and Cavaliere and Taylor (2008, Lemma 2) show that $\left\|\hat{\Phi}_{p}-\Phi_{p}\right\|=o_{p}\left(p^{-1 / 2}\right)$, where $\hat{\Phi}_{p}:=\left(\hat{\phi}_{p, 1}, \ldots, \hat{\phi}_{p, p}\right)^{\prime}$ and $\Phi_{p}:=\left(\phi_{1}, \ldots, \phi_{p}\right)^{\prime}$, using which the lag augmentation can be handled as in Chang and Park (2002, Lemma 3.1 and Lemma 3.2) and Smeekes (2009, Lemma 1). The result then follows. 
Proof of Theorem 2. The invariance principle for $\varepsilon_{t}^{*}$,

$$
T^{-1 / 2} \sum_{t=1}^{\lfloor T r\rfloor} \varepsilon_{t}^{*} \stackrel{d^{*}}{\longrightarrow} \bar{\omega} W_{\eta, 0}(r) \quad \text { in probability, }
$$

follows directly from the proof of Theorem 2, Equation (A.4), of Cavaliere and Taylor (2008). We next show that

$$
T^{-1 / 2} \sum_{t=1}^{\lfloor T r\rfloor} u_{t}^{*} \stackrel{d^{*}}{\longrightarrow} \bar{\omega} \psi(1) W_{\eta, 0}(r) \quad \text { in probability. }
$$

Letting $\hat{\Phi}(L):=1-\sum_{j=1}^{q} \hat{\phi}_{q, j} L^{j}$ and $\hat{\Psi}(1):=\hat{\Phi}(1)^{-1}$, we can write using the Beveridge-Nelson decomposition

$$
T^{-1 / 2} \sum_{t=1}^{\lfloor T r\rfloor} u_{t}^{*}=T^{-1 / 2} \sum_{t=1}^{\lfloor T r\rfloor} \hat{\Psi}(1) \varepsilon_{t}^{*}+T^{-1 / 2}\left(\bar{u}_{0}^{*}-\bar{u}_{\lfloor T r\rfloor}^{*}\right),
$$

where $\bar{u}_{t}^{*}:=\hat{\Psi}(1) \sum_{i=1}^{q}\left(\sum_{j=i}^{q} \hat{\Phi}_{j}\right) u_{t-i+1}^{*}$. As $\left\|\hat{\Phi}_{q}-\Phi_{q}\right\|=o_{p}\left(k^{-1 / 2}\right)$, it follows directly that $\hat{\Psi}(1) \stackrel{p}{\rightarrow} \Psi(1)$. Then (20) follows if we can show that $\mathrm{P}^{*}\left\{\max _{1 \leq t \leq T}\left|T^{-1 / 2} \bar{u}_{t}^{*}\right|>\epsilon\right\}=o_{p}(1)$. As in Chang and Park (2002), we may write for large $T$ that $u_{t}^{*}=\sum_{j=0}^{\infty} \hat{\psi}_{j} \varepsilon_{t-j}^{*}$ and furthermore $\bar{u}_{t}^{*}=\sum_{j=0}^{\infty} \bar{\psi}_{j} \varepsilon_{t-j}^{*}$, where $\bar{\psi}_{j}:=\sum_{i=j+1}^{\infty} \hat{\psi}_{j}$. Then, as in Cavaliere and Taylor (2007, Eq. (14)), this holds if $\sup _{t} \mathrm{E}\left(\bar{u}_{t}^{4}\right)=O_{p}(1)$. Then, as $\varepsilon_{t}^{*}=\hat{\varepsilon}_{t} \xi_{t}^{*}$, by the MarcinkiewiczZygmund inequality and Minkowski's inequality we have that

$$
\begin{aligned}
T^{-1} \sup _{t} \mathrm{E}^{*}\left(\bar{u}_{t}^{* 4}\right) & =\sup _{t} T^{-1} \mathrm{E}^{*}\left(\sum_{j=0}^{\infty} \bar{\psi}_{j}^{*} \varepsilon_{t-j}^{*}\right)^{4} \leq c T^{-1} \sup _{t} \mathrm{E}^{*}\left(\sum_{j=0}^{\infty} \bar{\psi}_{j}^{* 2} \varepsilon_{t-j}^{* 2}\right)^{2} \\
& \leq c T^{-1} \sup _{t}\left\{\sum_{j=0}^{\infty}\left[\mathrm{E}^{*}\left(\bar{\psi}_{j}^{* 4} \varepsilon_{t-j}^{* 4}\right)\right]^{1 / 2}\right\}^{2} \\
& \leq \sqrt{3} c\left(\sum_{j=0}^{\infty} \bar{\psi}_{j}^{* 2}\right)^{2}\left(T^{-1 / 2} \sup _{t} \hat{\varepsilon}_{q, t-j}^{2}\right)^{2}
\end{aligned}
$$

As $\sum_{j=1}^{q} j^{1 / 2}\left|\hat{\phi}_{q, j}\right|=O_{p}(1)$ (by Assumption 1(i) and $\left\|\hat{\Phi}_{q}-\phi_{q}\right\|=o_{p}\left(k^{-1 / 2}\right)$ ), it follows from Phillips and Solo (1992) that $\sum_{j=0}^{\infty} \bar{\psi}_{j}^{* 2}=O_{p}(1)$. Furthermore, $T^{-1 / 2} \sup _{t} \hat{\varepsilon}_{q, t-j}^{2} \leq$ $T^{-1 / 2} \sum_{t=1}^{T} \hat{\varepsilon}_{q, t-j}^{2}=O_{p}(1)$ by Cavaliere and Taylor (2008, Lemma 2). Therefore we can conclude that (20) holds.

It then follows straightforwardly for case (i) and (ii) that

$$
T^{-1 / 2} \hat{x}_{\lfloor T r\rfloor, \gamma}^{\delta *} \stackrel{d^{*}}{\longrightarrow} K_{\eta, 0, \gamma}^{\delta}(r) \quad \text { in probability, }
$$


while for case (iii) it follows along the same lines as in the proof of Theorem 1 that

$$
T^{-1 / 2} \hat{x}_{\lfloor T r\rfloor, \gamma}^{\mu *} \stackrel{d^{*}}{\longrightarrow} K_{\eta, c, \gamma, \tilde{\gamma}}^{\mu *}(r) \quad \text { in probability. }
$$

Finally, the lag augmentation can again be handled as in Chang and Park (2003, Theorem 2) and Smeekes (2009, Lemma 5) to find the limiting distributions of the $D F-\gamma^{\delta *}$ statistics. 


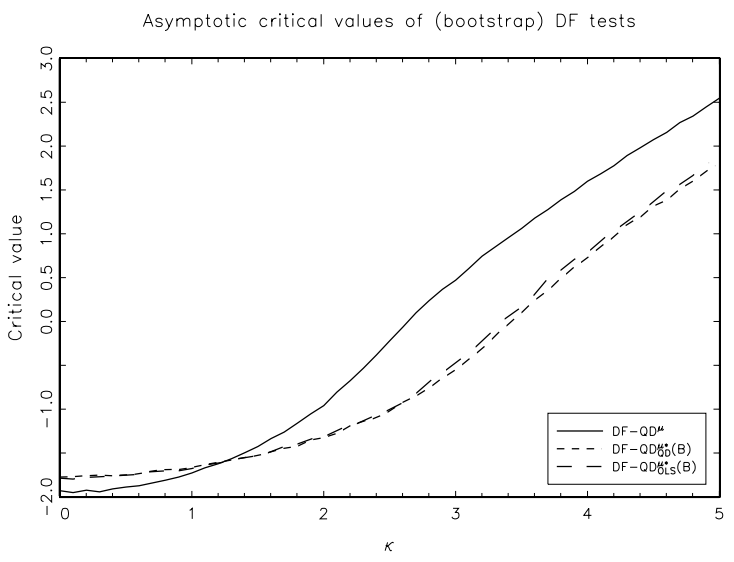

(a) DF-QD

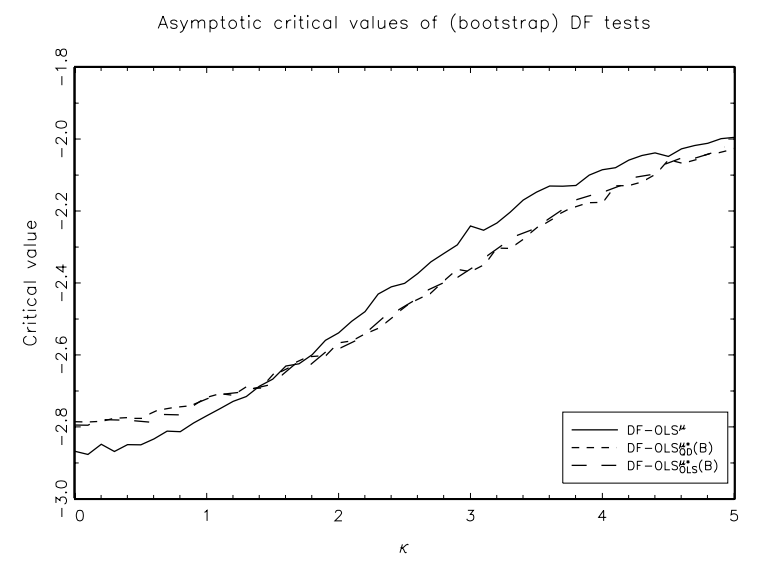

(b) DF-OLS

Figure 1: Critical values DF tests for varying $\kappa$ 
Asymptotic size of $U_{R_{4}}$ tests

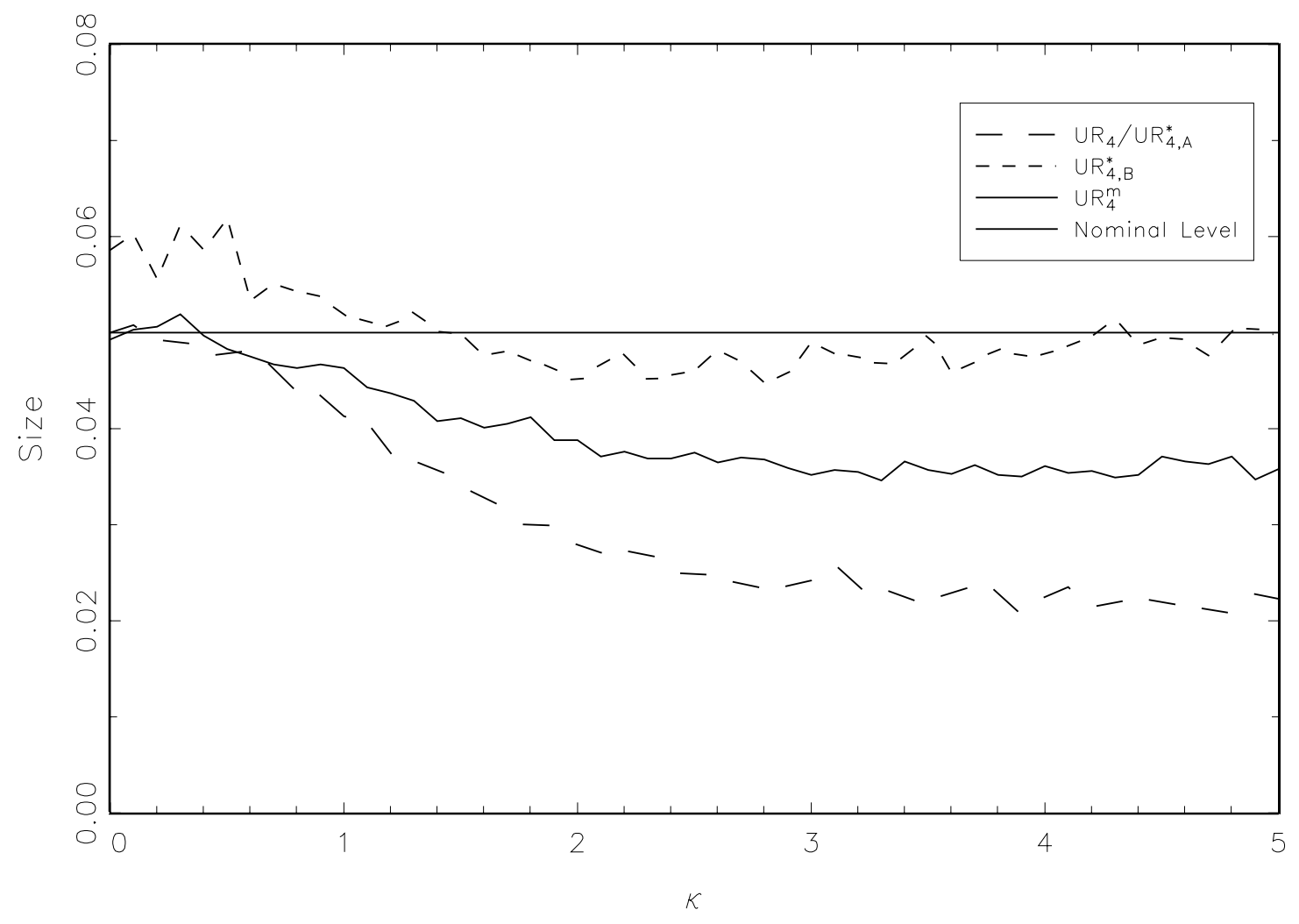

Figure 2: Asymptotic size 
Asymototic power of UR

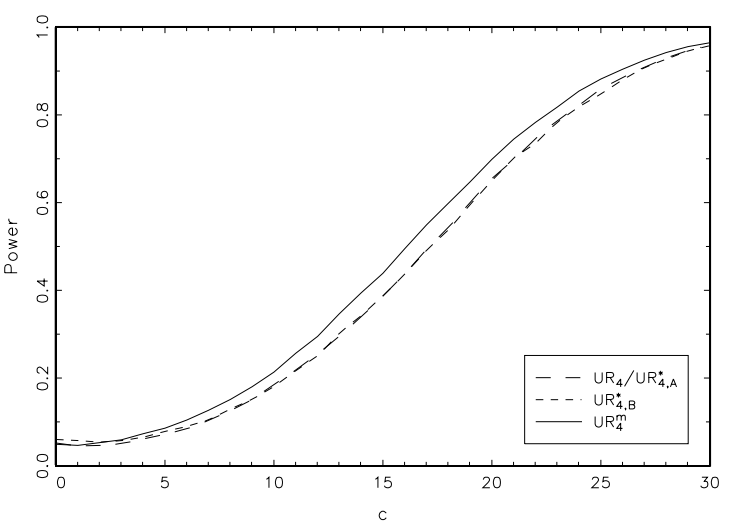

(a) $\alpha=-2$

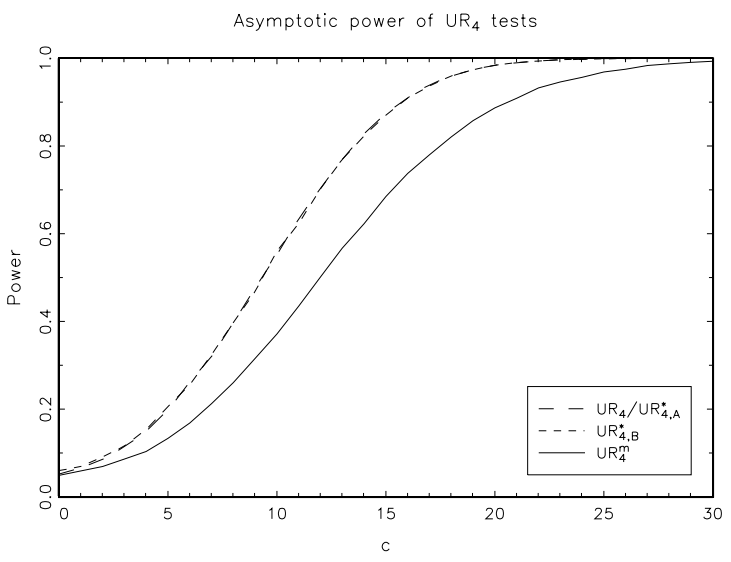

(c) $\alpha=0$

Asymptotic power of UR 4 tests

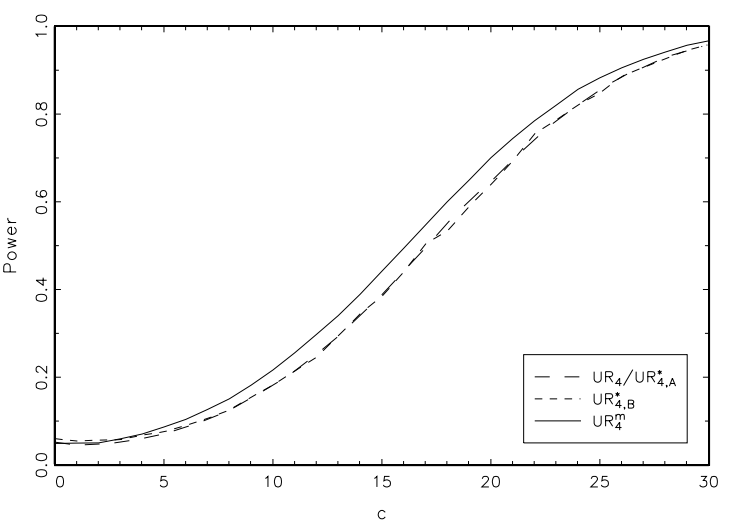

(e) $\alpha=2$
Asymptotic power of UR tests

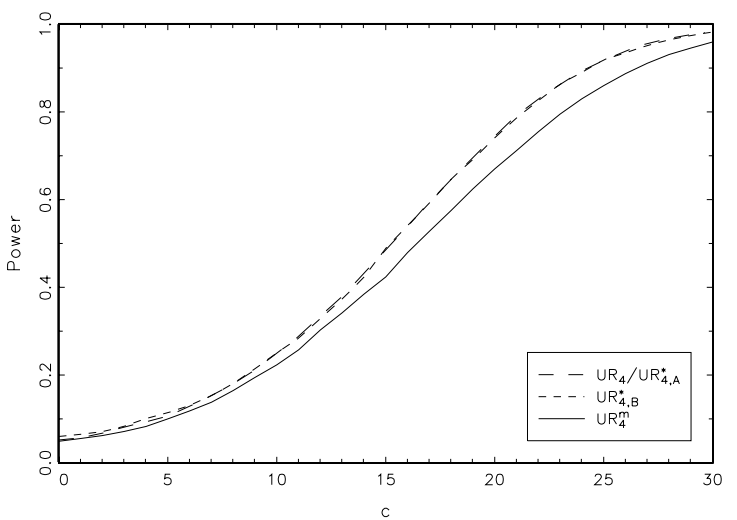

(b) $\alpha=-1$

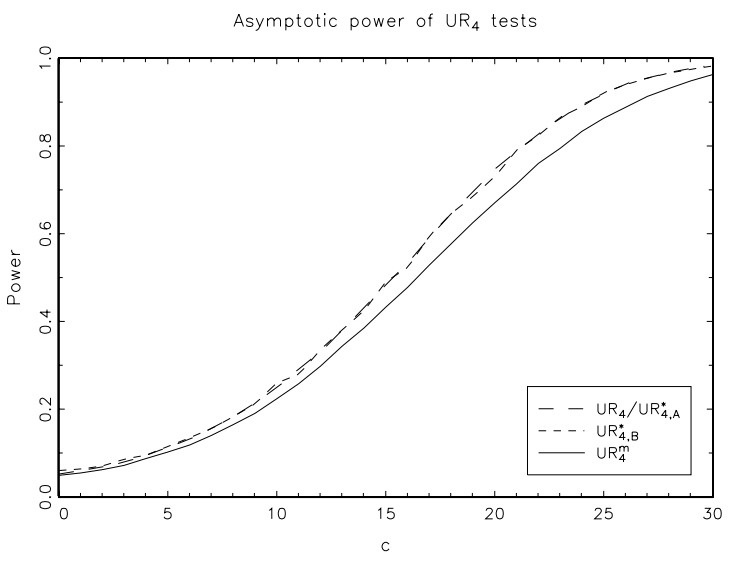

(d) $\alpha=1$

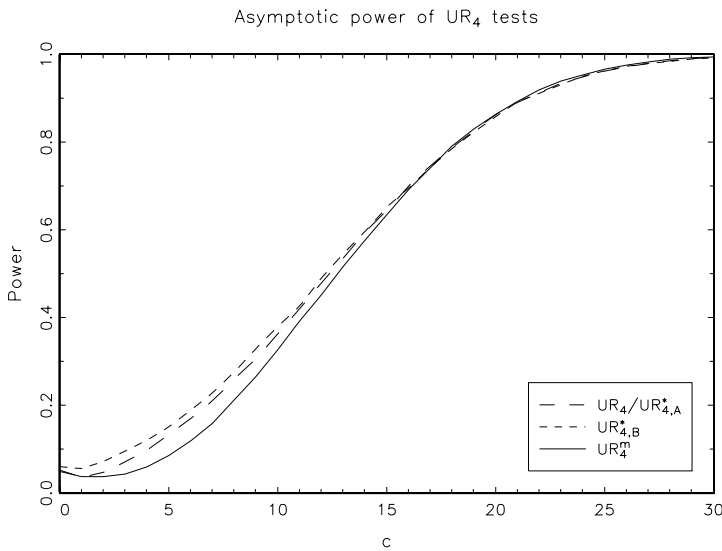

(f) $\alpha=4$

Figure 3: Asymptotic power for $\kappa=0$ 
Asymototic power of UR

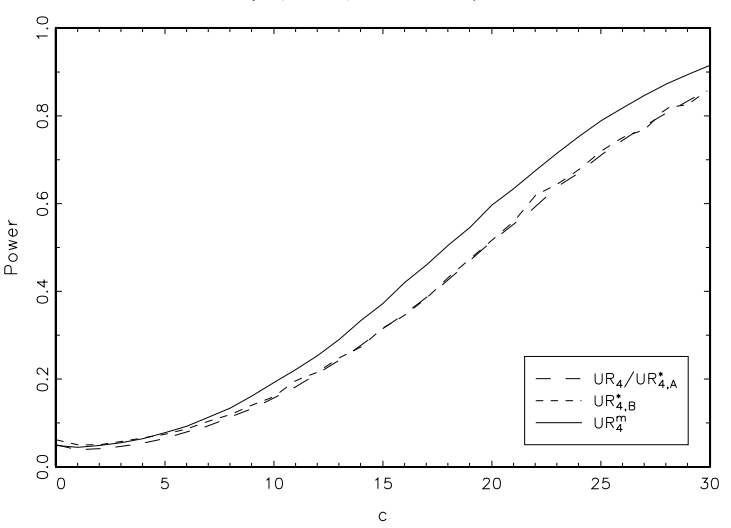

(a) $\alpha=-2$

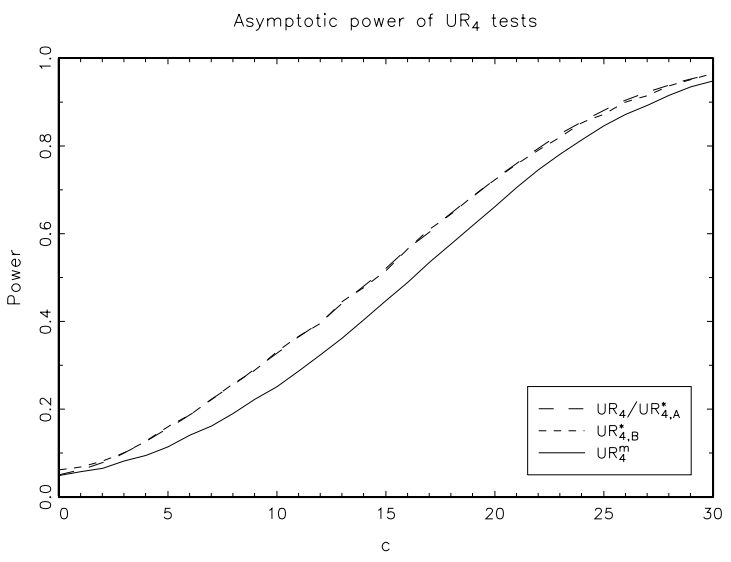

(c) $\alpha=0$

Asymototic power of $\mathrm{UR}_{4}$ tests

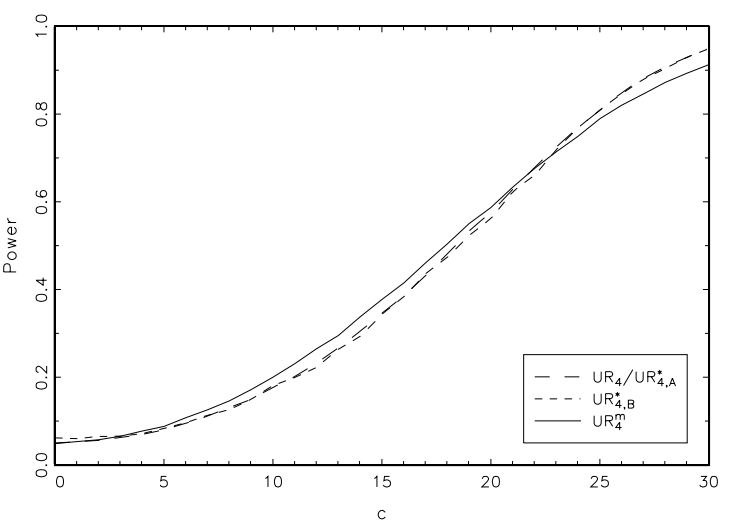

(e) $\alpha=2$
Asymptotic power of UR tests

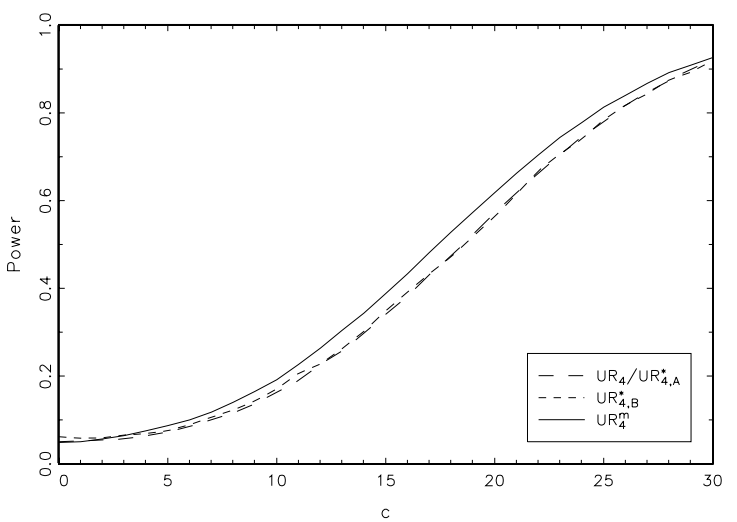

(b) $\alpha=-1$

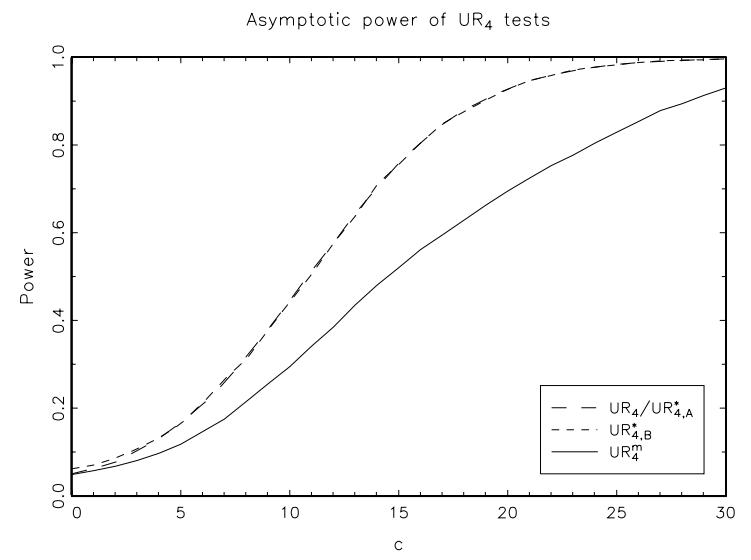

(d) $\alpha=1$

Asymptotic power of $U R_{4}$ tests

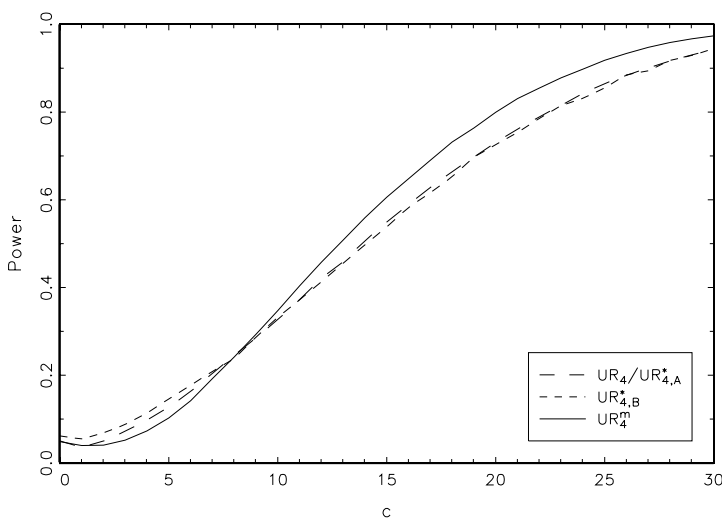

(f) $\alpha=4$

Figure 4: Asymptotic power for $\kappa=0.25$ 
Asymototic power of UR

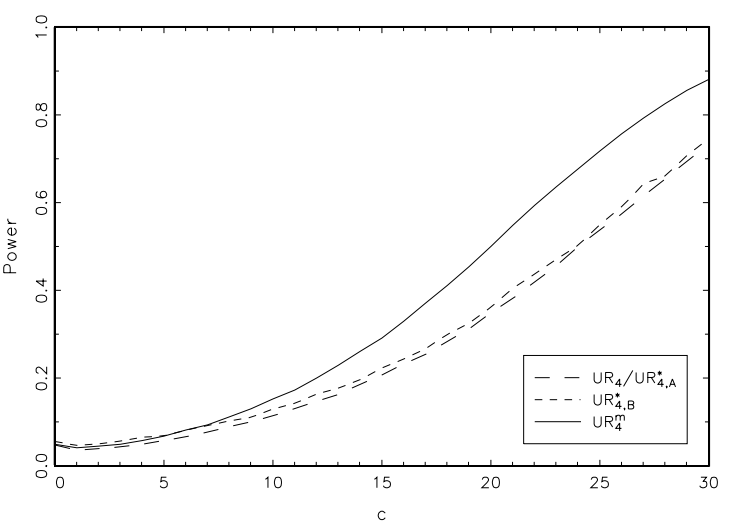

(a) $\alpha=-2$

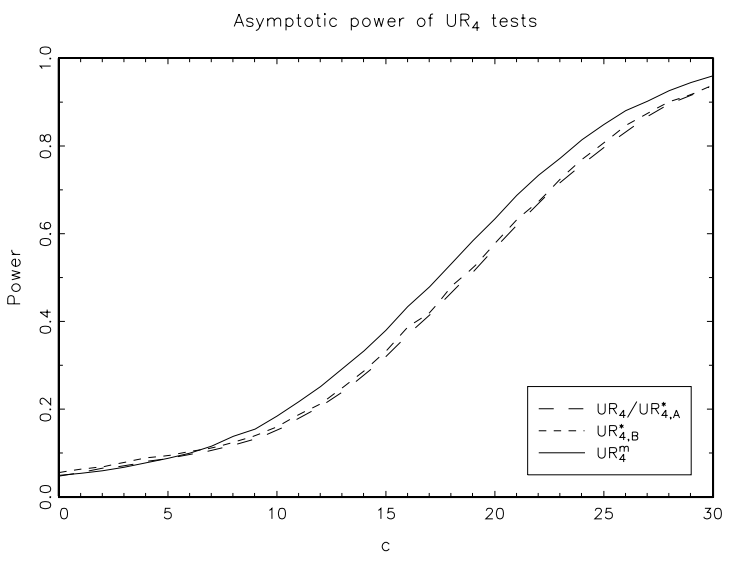

(c) $\alpha=0$

Asymptotic power of UR 4 tests

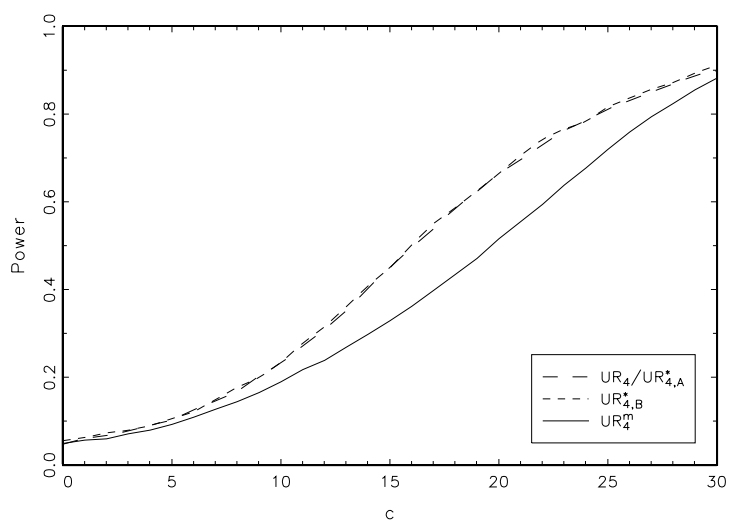

(e) $\alpha=2$
Asymptotic power of UR tests

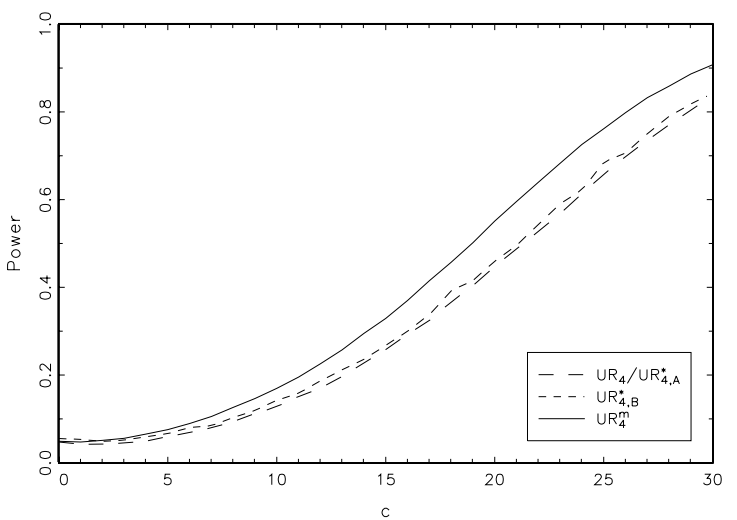

(b) $\alpha=-1$

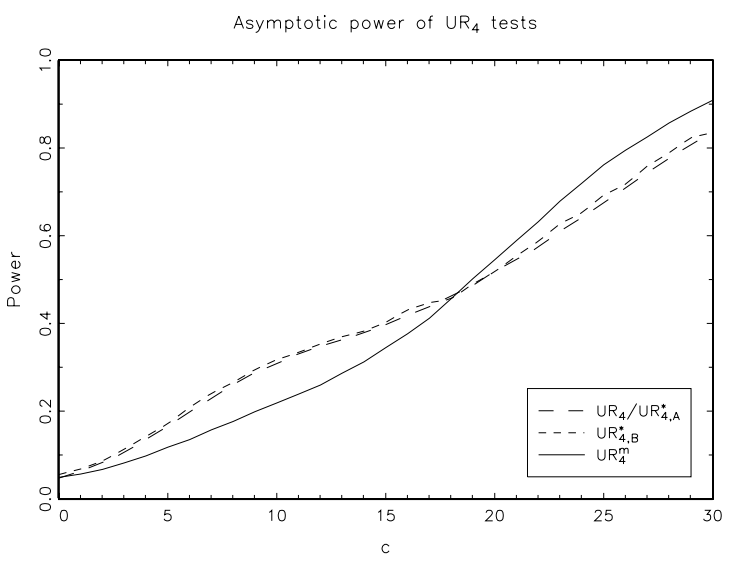

(d) $\alpha=1$

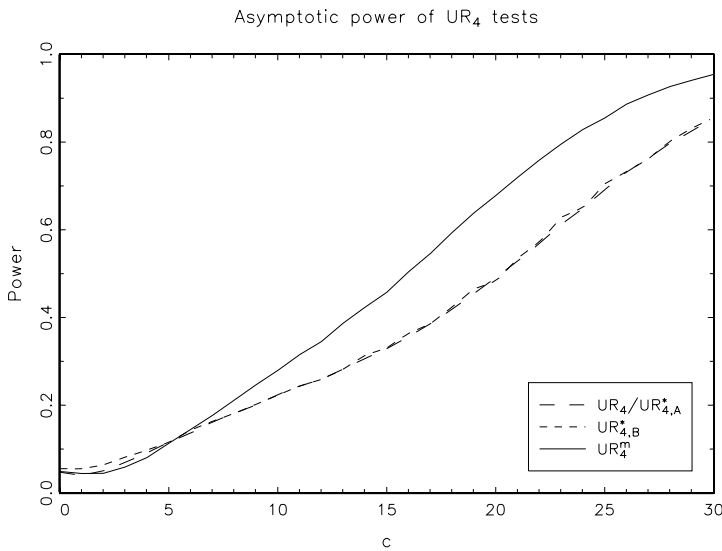

(f) $\alpha=4$

Figure 5: Asymptotic power for $\kappa=0.5$ 
Asymototic power of UR

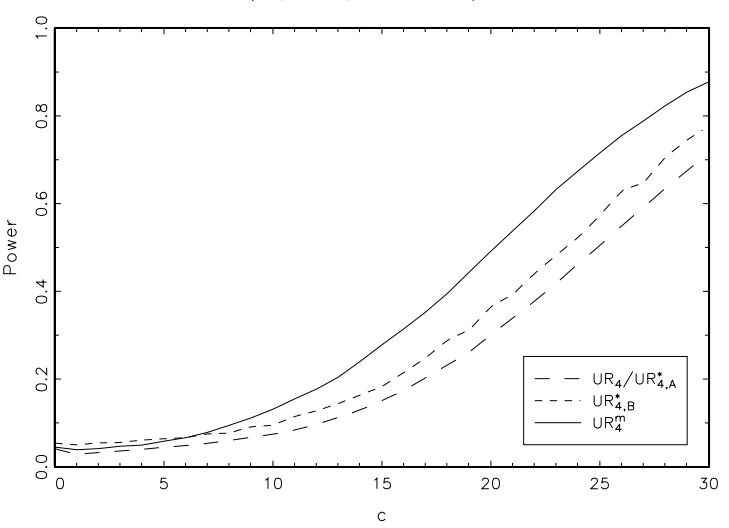

(a) $\alpha=-2$

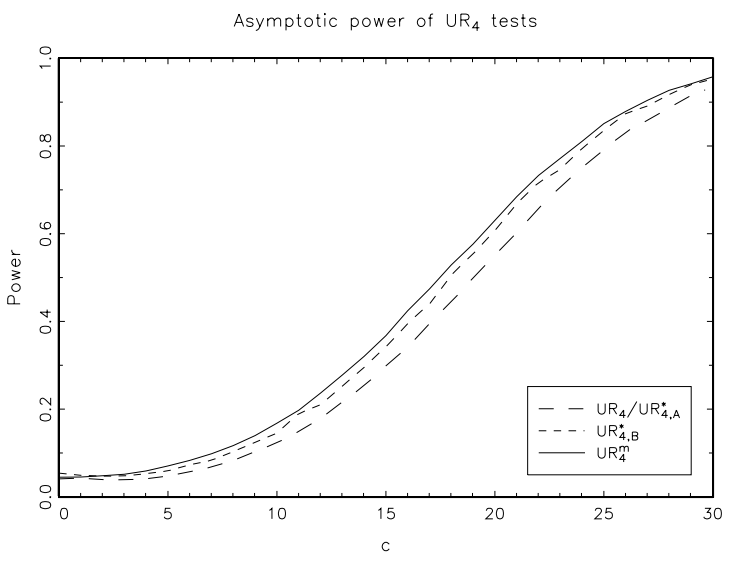

(c) $\alpha=0$

Asymototic power of $\mathrm{UR}_{4}$ tests

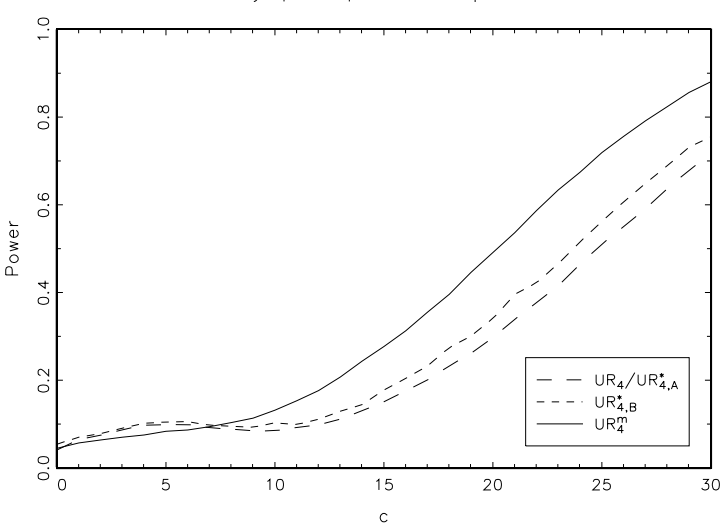

(e) $\alpha=2$
Asymptotic power of UR tests

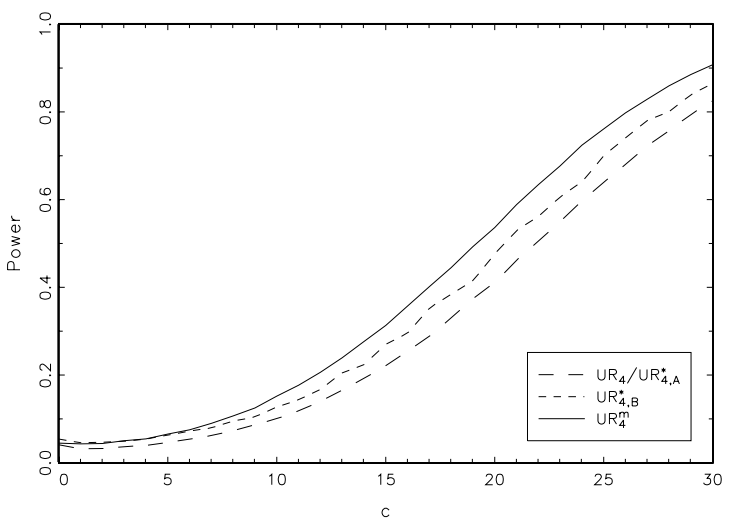

(b) $\alpha=-1$

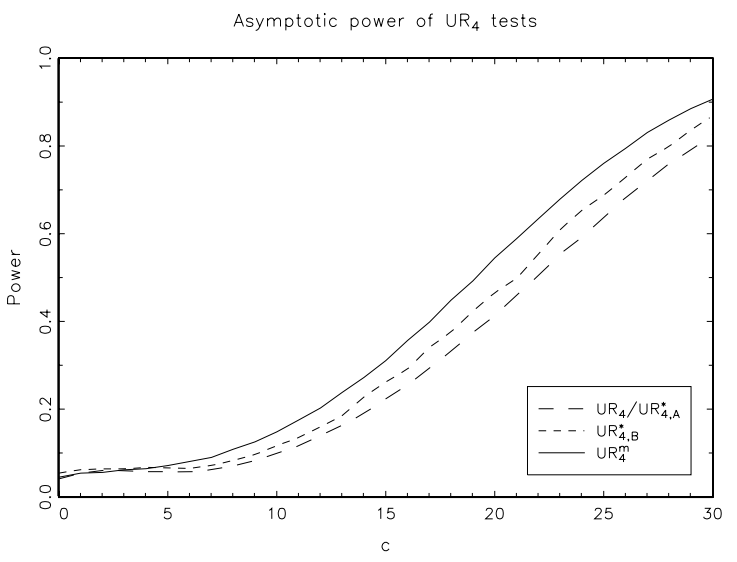

(d) $\alpha=1$

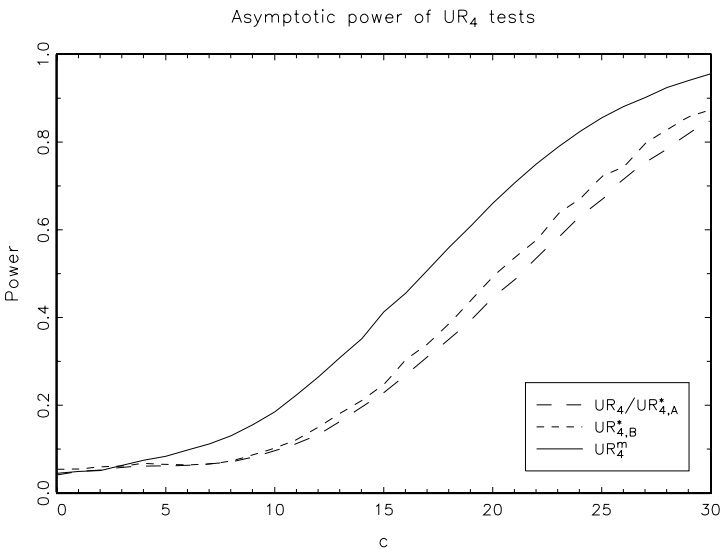

(f) $\alpha=4$

Figure 6: Asymptotic power for $\kappa=1$ 
Asymototic power of UR

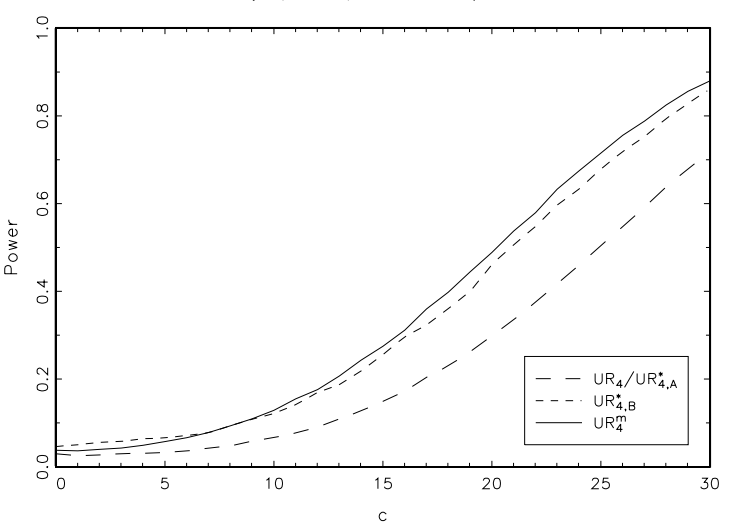

(a) $\alpha=-2$

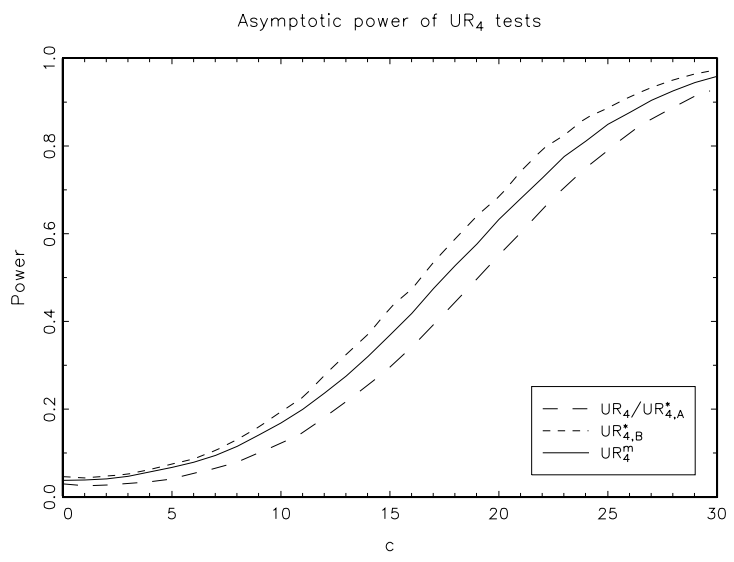

(c) $\alpha=0$

Asymototic power of UR 4 tests

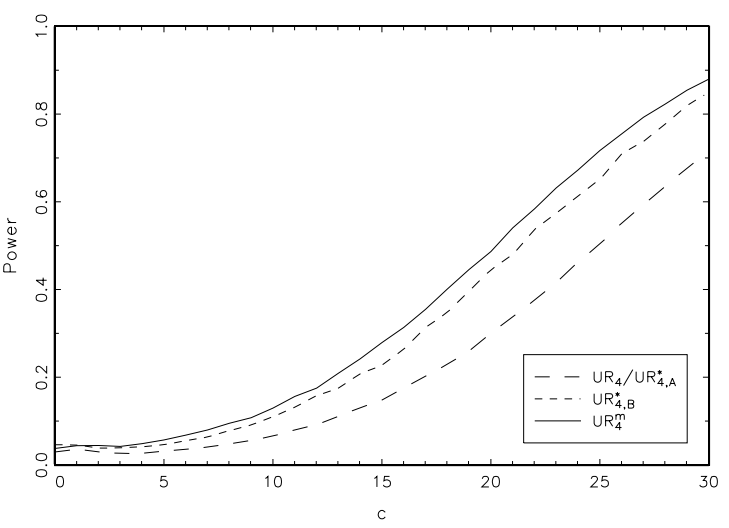

(e) $\alpha=2$
Asymptotic power of $U R_{2}$ tests

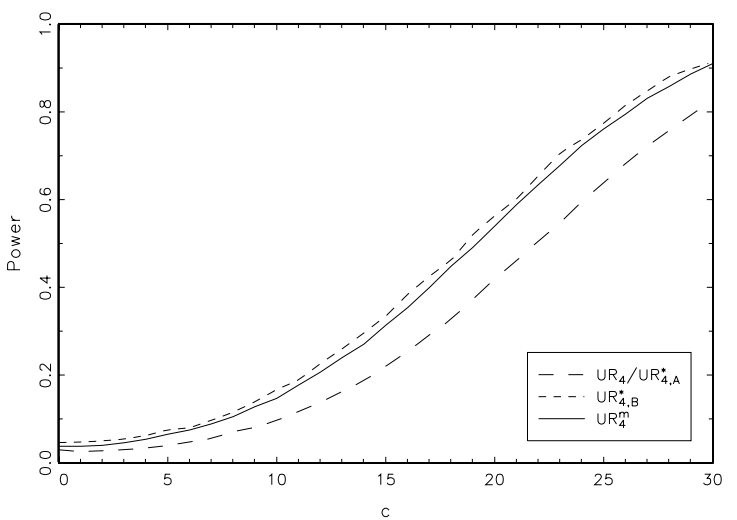

(b) $\alpha=-1$

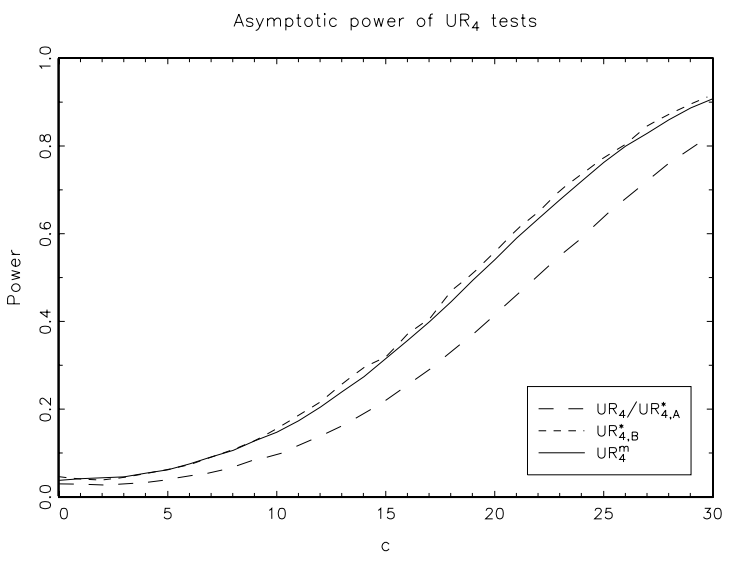

(d) $\alpha=1$

Asymptotic power of $U R_{4}$ tests

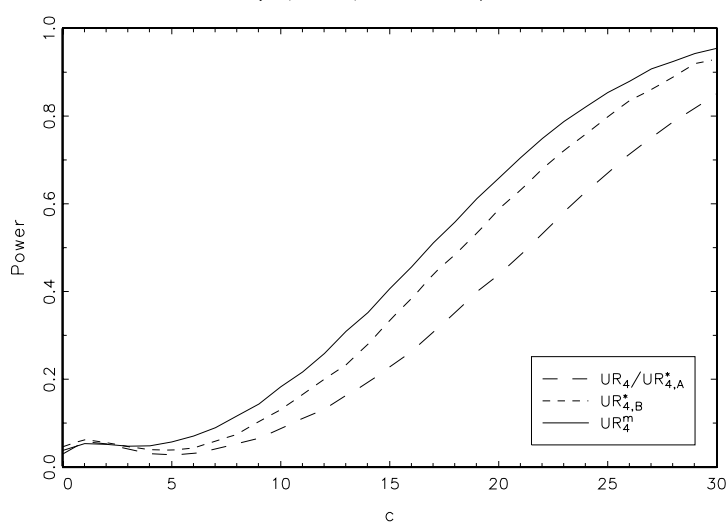

(f) $\alpha=4$

Figure 7: Asymptotic power for $\kappa=2$ 
Asymototic power of UR

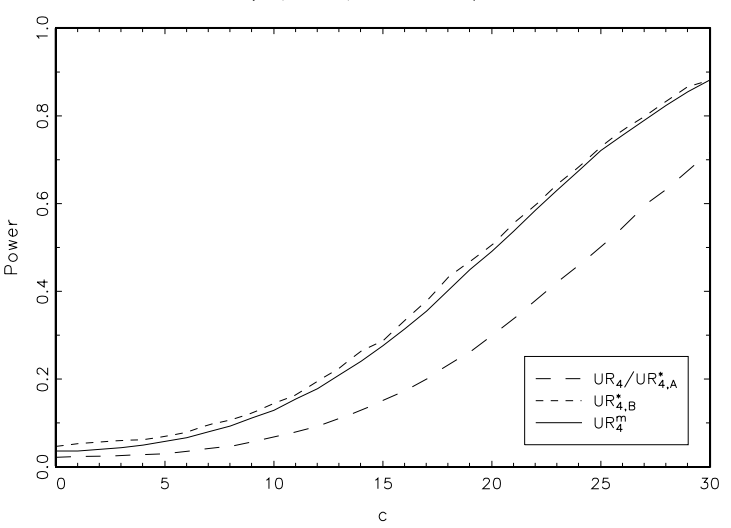

(a) $\alpha=-2$

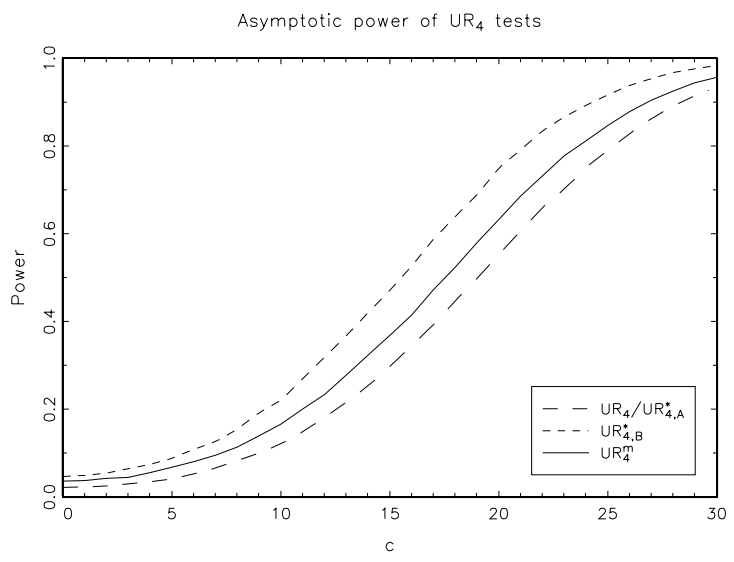

(c) $\alpha=0$

Asymototic power of UR 4 tests

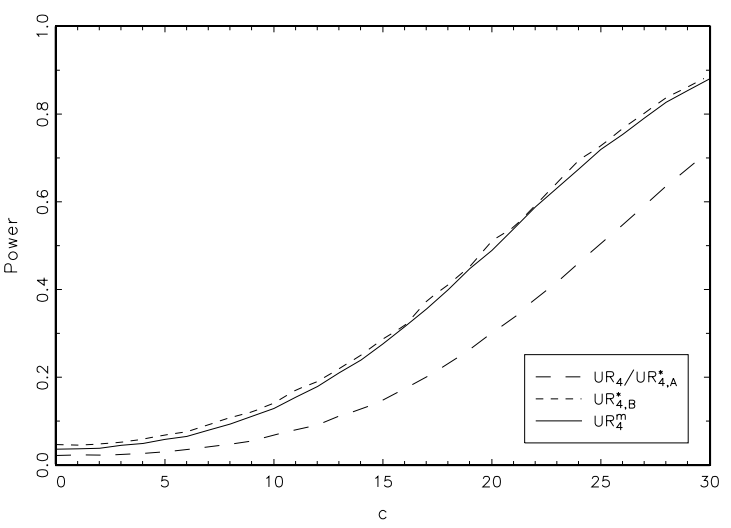

(e) $\alpha=2$
Asymptotic power of UR tests

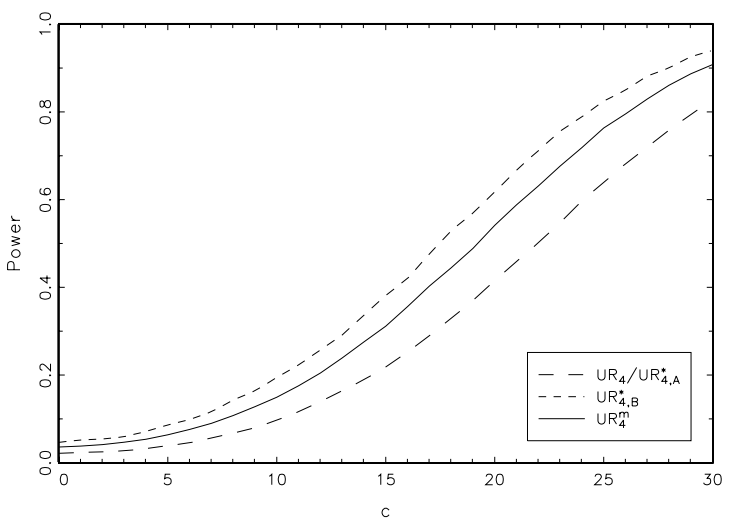

(b) $\alpha=-1$

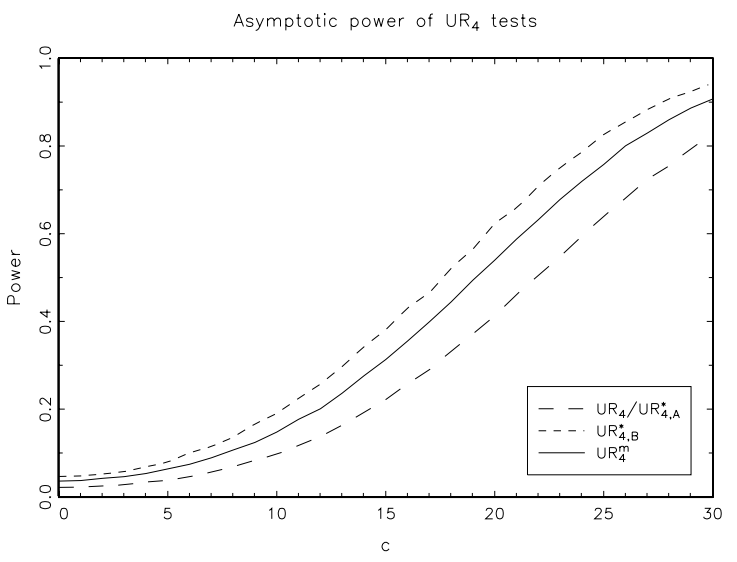

(d) $\alpha=1$

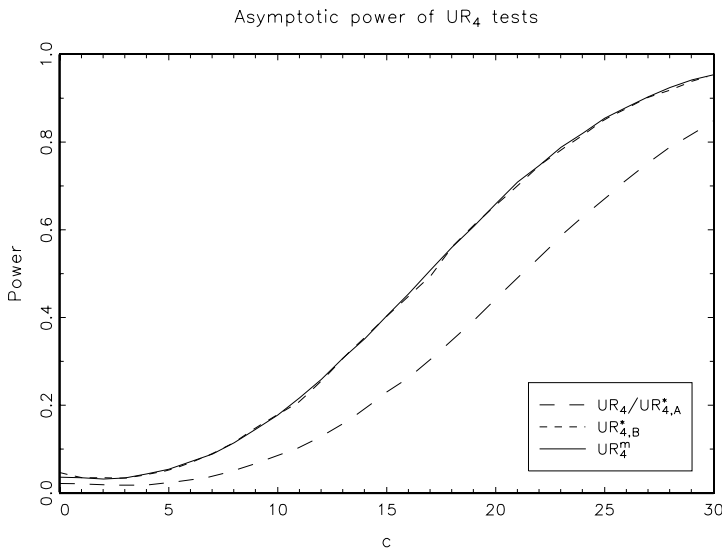

(f) $\alpha=4$

Figure 8: Asymptotic power for $\kappa=4$ 


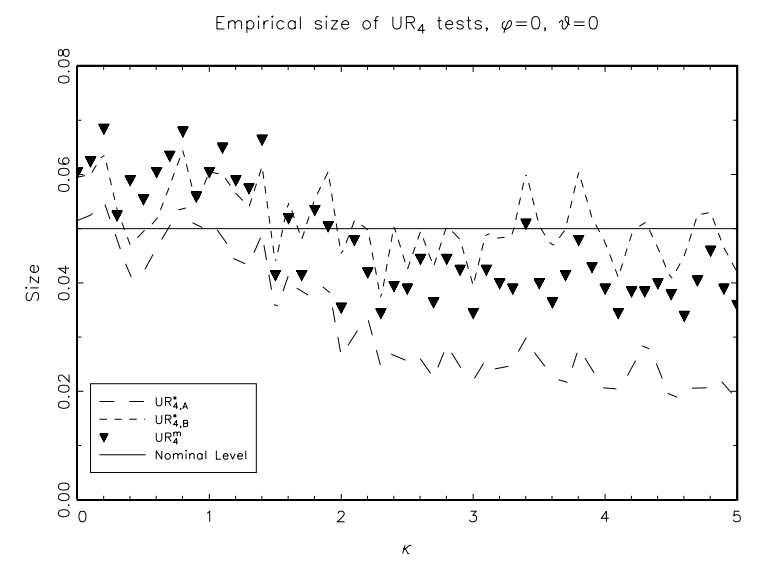

(a) $\varphi=0$ and $\vartheta=0$

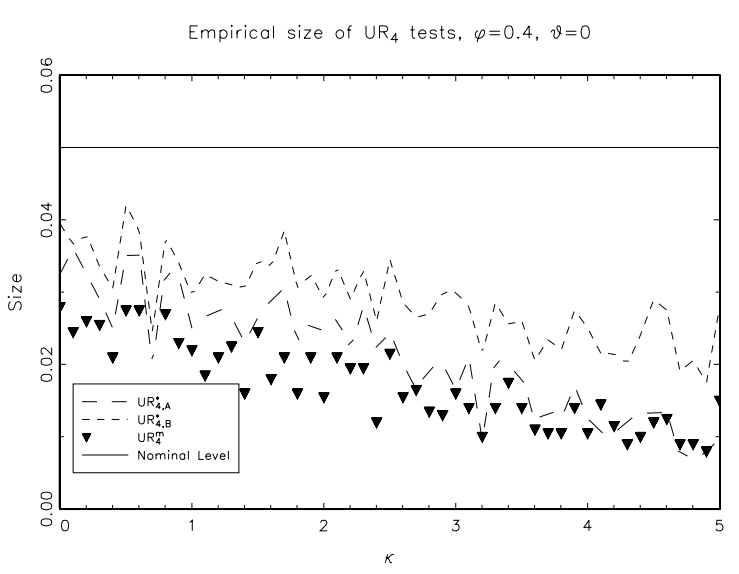

(b) $\varphi=0.4$ and $\vartheta=0$

Empirical size of $\mathrm{UR}_{4}$ tests, $\varphi=0, v=0.4$

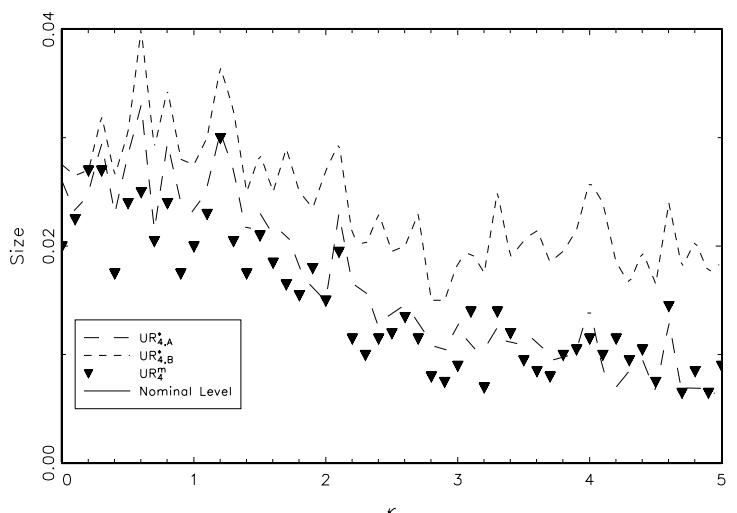

(d) $\varphi=0$ and $\vartheta=0.4$

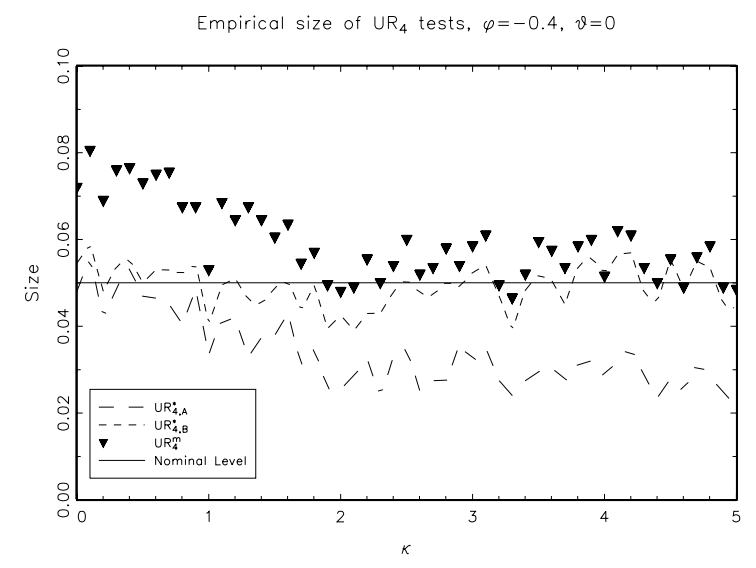

(c) $\varphi=-0.4$ and $\vartheta=0$

Empirical size of $\cup R_{4}$ tests, $\varphi=0, v=-0.4$

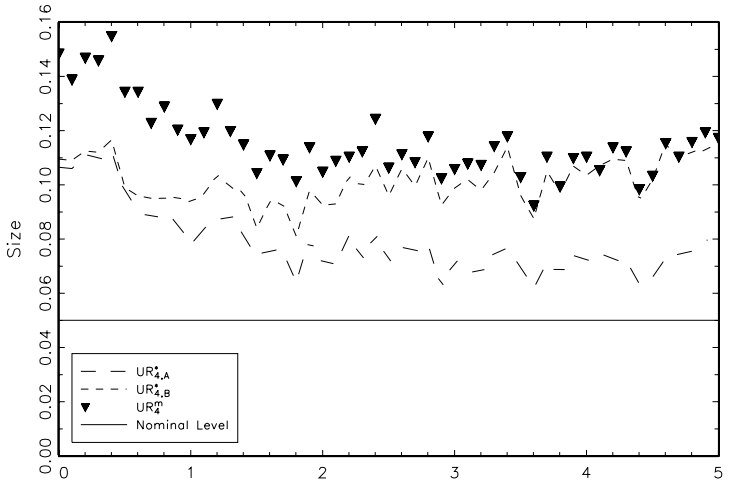

(e) $\varphi=0$ and $\vartheta=-0.4$

Figure 9: Small sample size $U R$ tests for varying $\kappa$ 


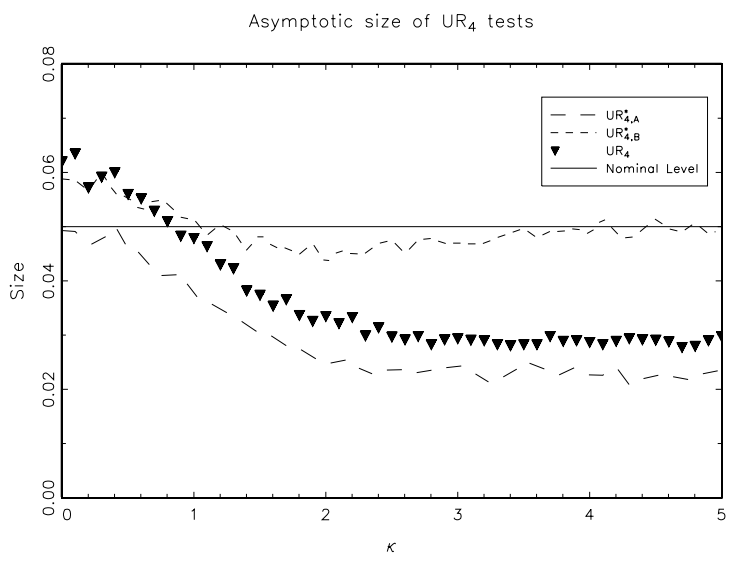

(a) Single break: $\sigma_{0} / \sigma_{1}=0.2$ and $\tau=0.9$

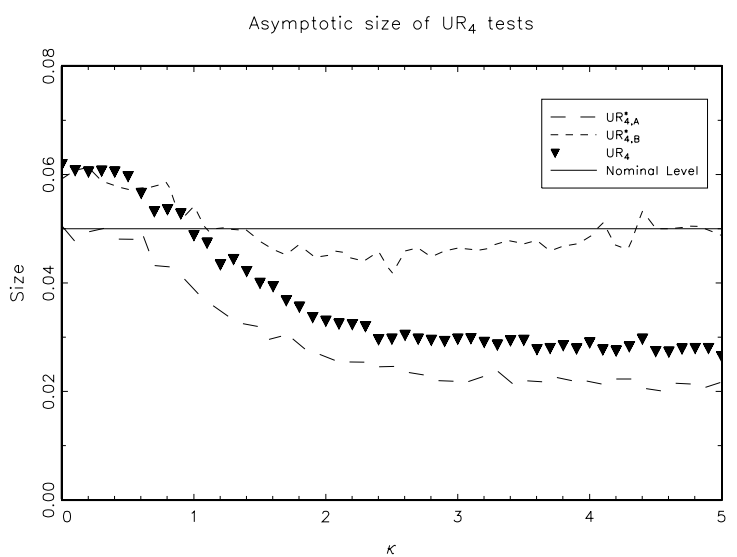

(c) Double break: $\sigma_{0} / \sigma_{1}=0.2$ and $\tau=0.45$

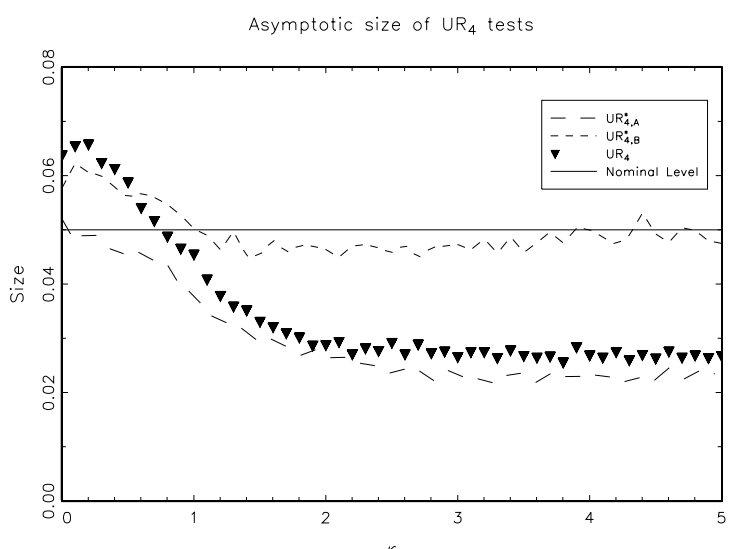

(e) Trending: $\sigma_{0} / \sigma_{1}=0.2$

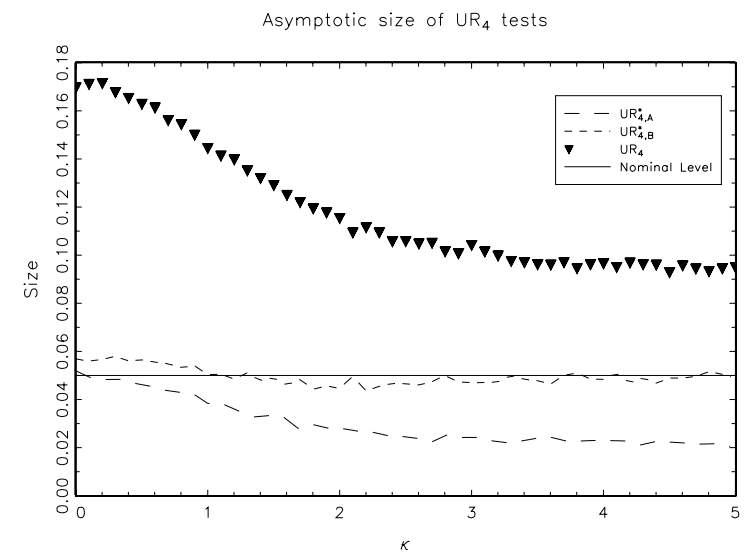

(b) Single break: $\sigma_{0} / \sigma_{1}=5$ and $\tau=0.1$

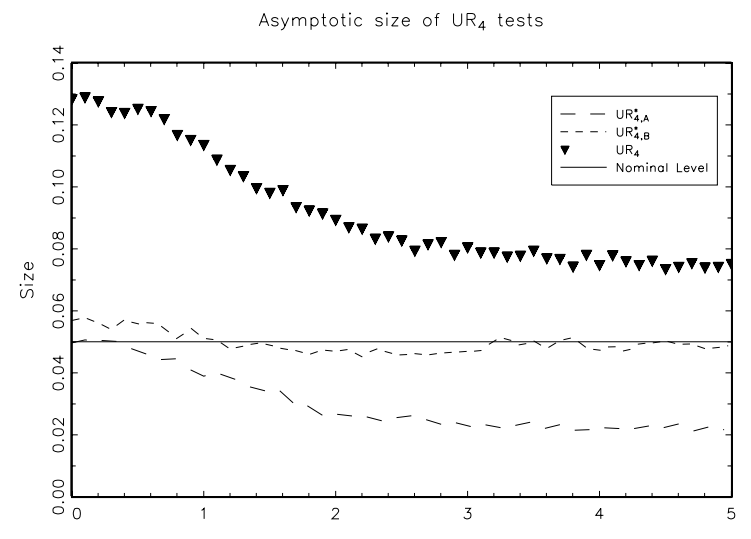

(d) Double break: $\sigma_{0} / \sigma_{1}=5$ and $\tau=0.05$

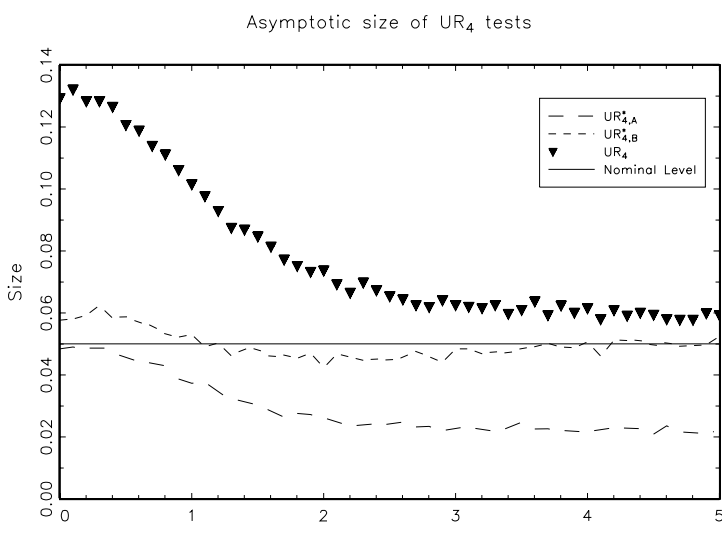

(f) Trending: $\sigma_{0} / \sigma_{1}=5$

Figure 10: Asymptotic size $U R$ tests for varying $\kappa$ 
Asymototic power of UR tests

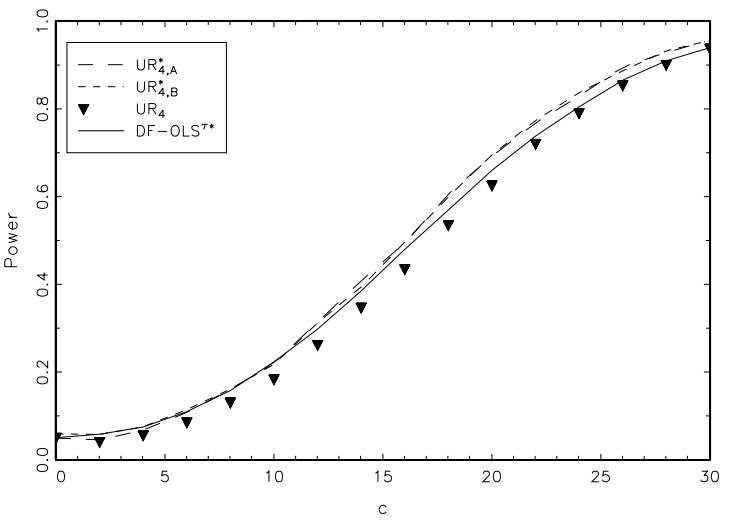

(a) $\alpha=-2$

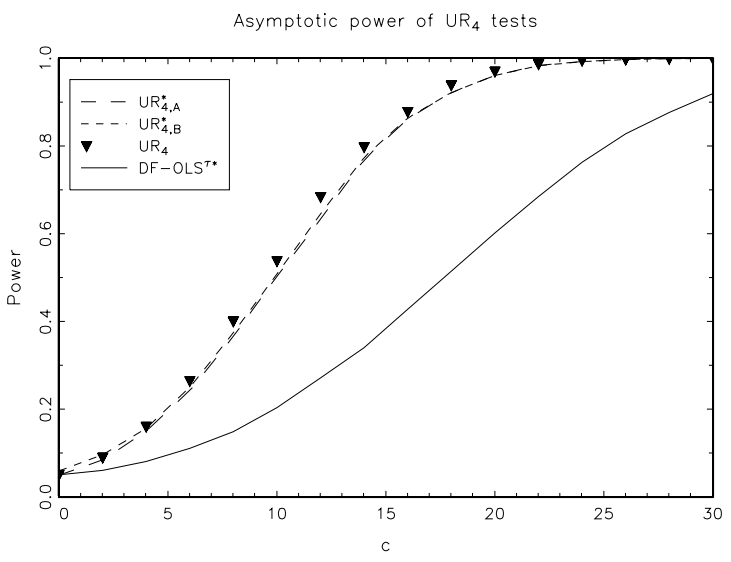

(c) $\alpha=0$

Asymototic power of $\mathrm{UR}_{4}$ tests

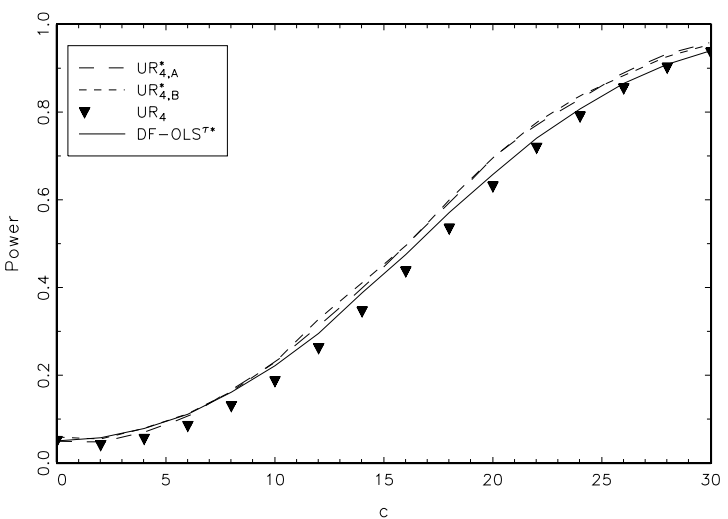

(e) $\alpha=2$
Asymptotic power of $u R_{\text {a tests }}$

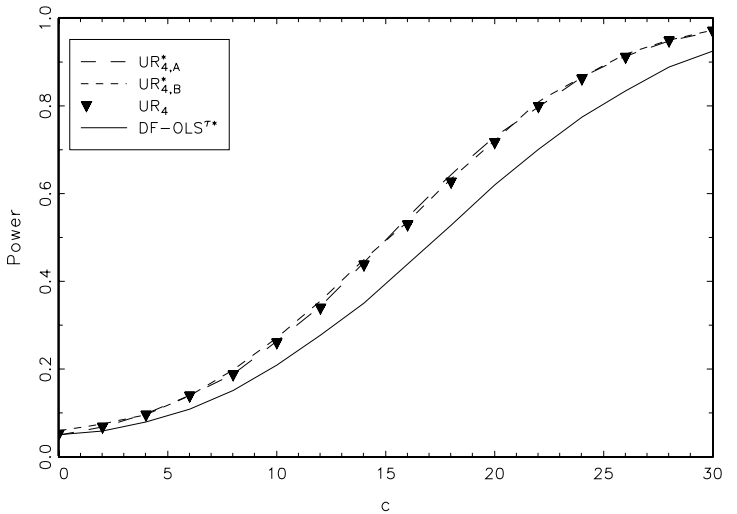

(b) $\alpha=-1$

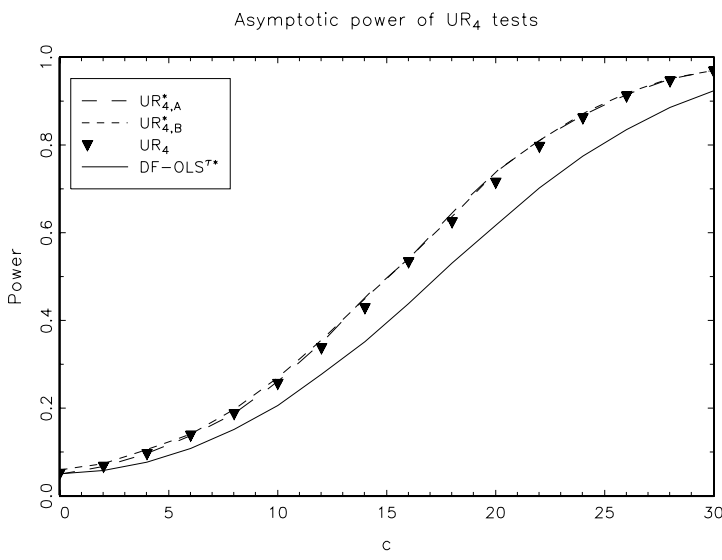

(d) $\alpha=1$

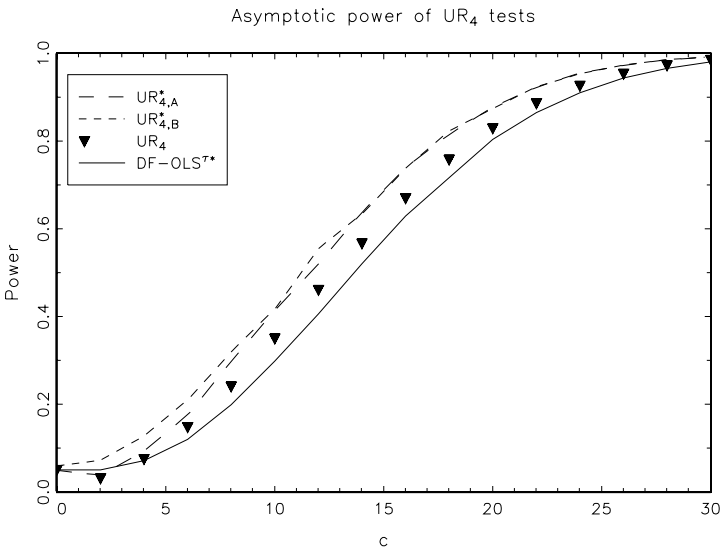

(f) $\alpha=4$

Figure 11: Asymptotic power for $\kappa=0$; Single break: $\sigma_{0} / \sigma_{1}=0.2$ and $\tau=0.9$ 
Asymototic power of UR testo

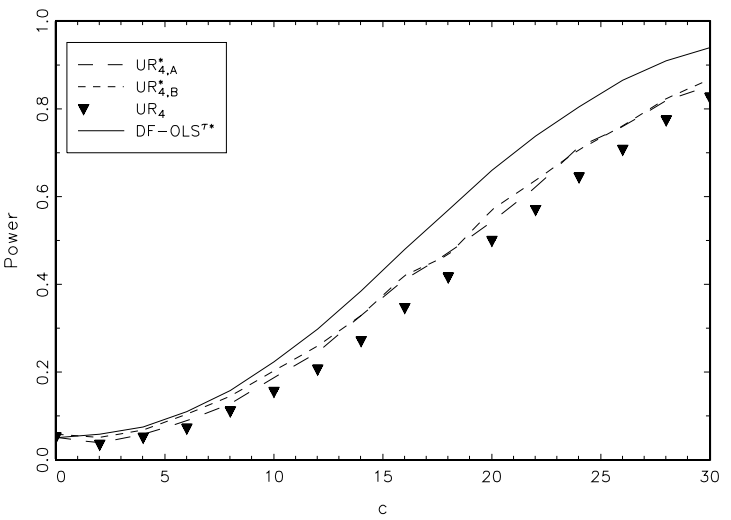

(a) $\alpha=-2$

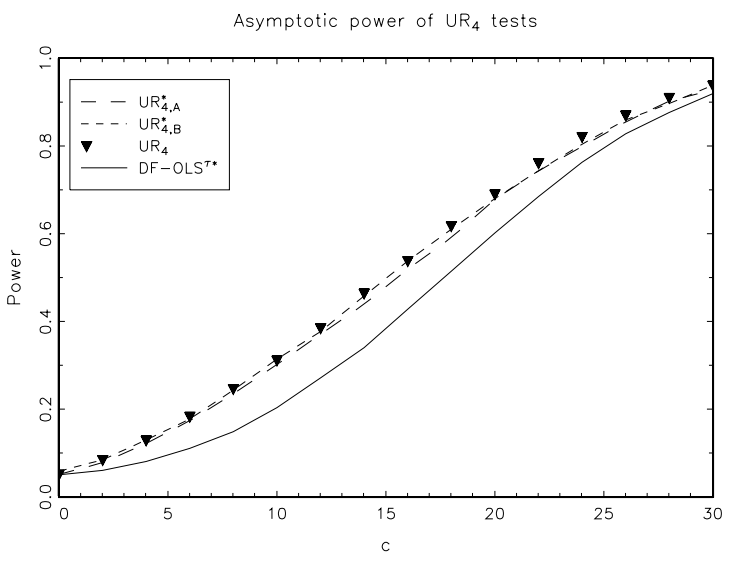

(c) $\alpha=0$

Asymototic power of $\mathrm{UR}_{4}$ tests

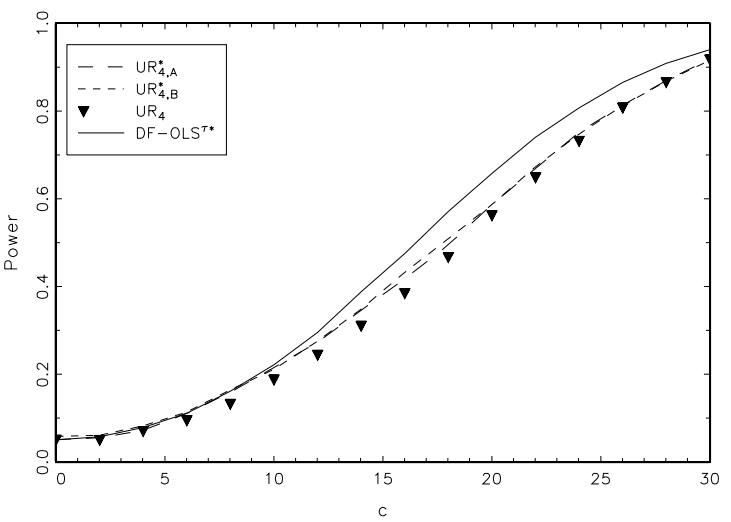

(e) $\alpha=2$
Asymptotic power of UR tests

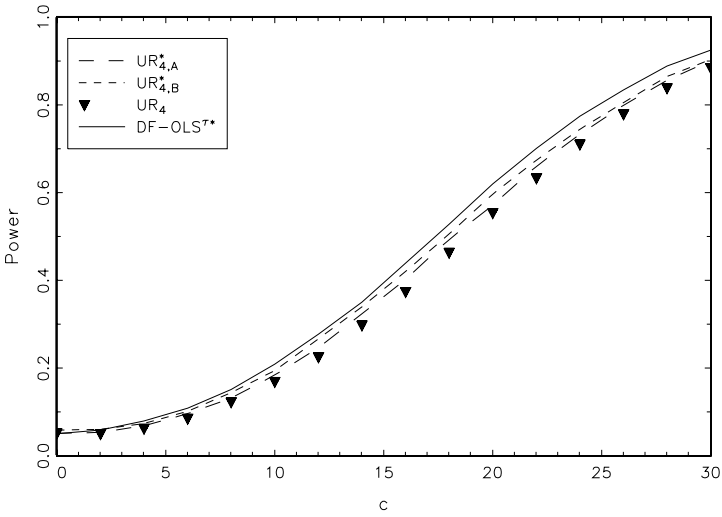

(b) $\alpha=-1$

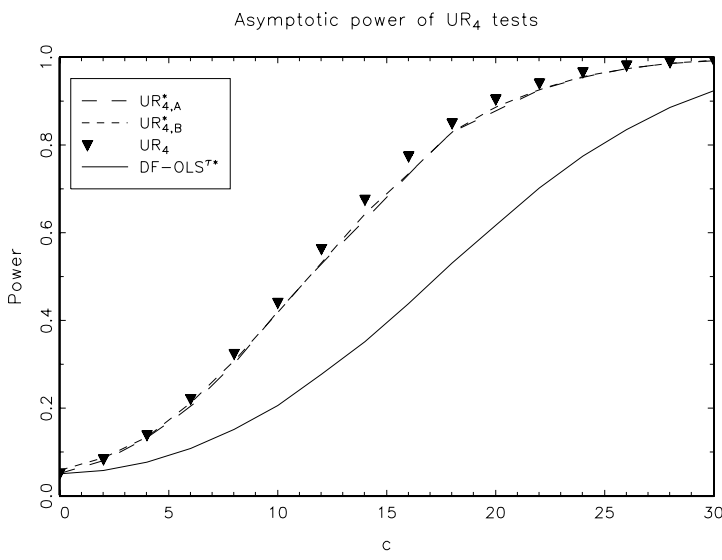

(d) $\alpha=1$

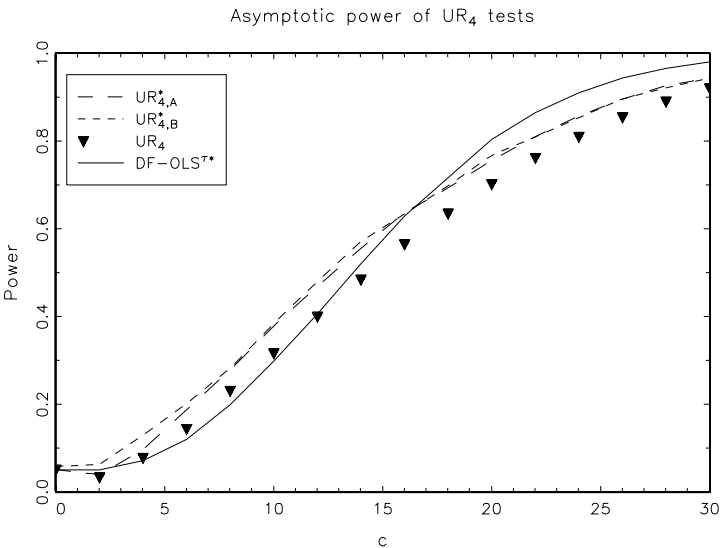

(f) $\alpha=4$

Figure 12: Asymptotic power for $\kappa=0.25$; Single break: $\sigma_{0} / \sigma_{1}=0.2$ and $\tau=0.9$ 
Acymototic power of UR testo

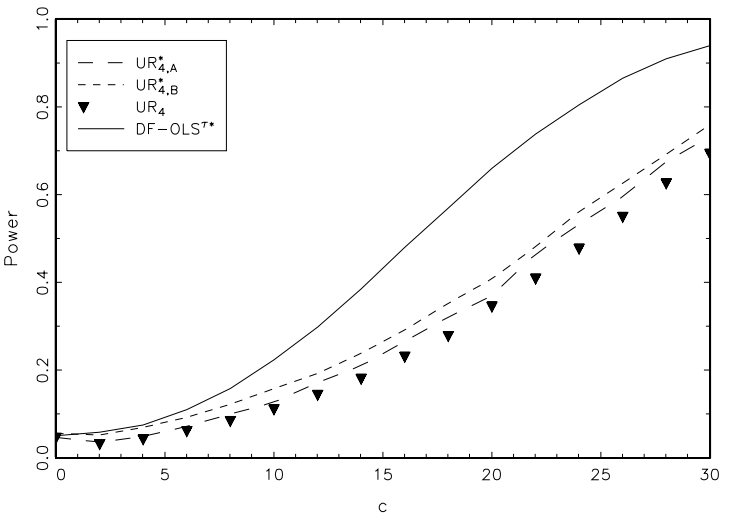

(a) $\alpha=-2$

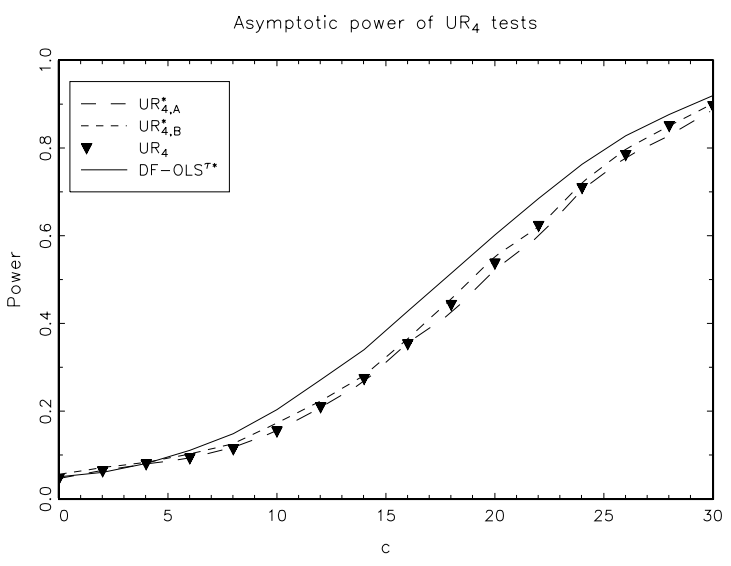

(c) $\alpha=0$

Asymototic power of $\mathrm{UR}_{4}$ tests

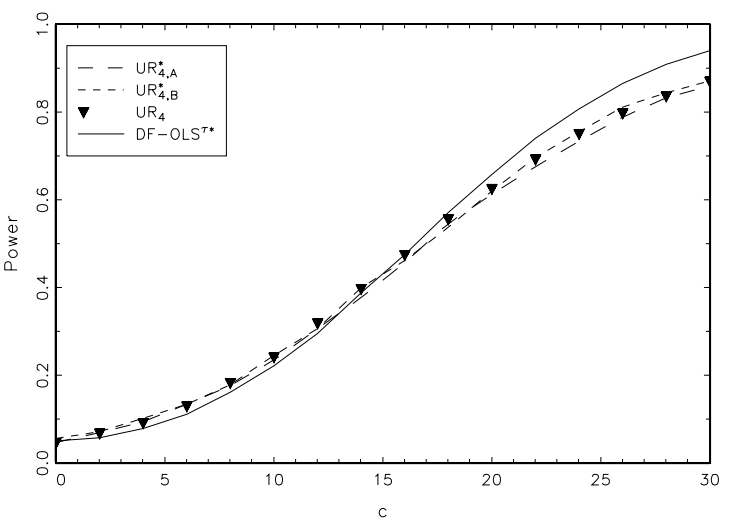

(e) $\alpha=2$
Asymptotic power of $u R_{\text {a tests }}$

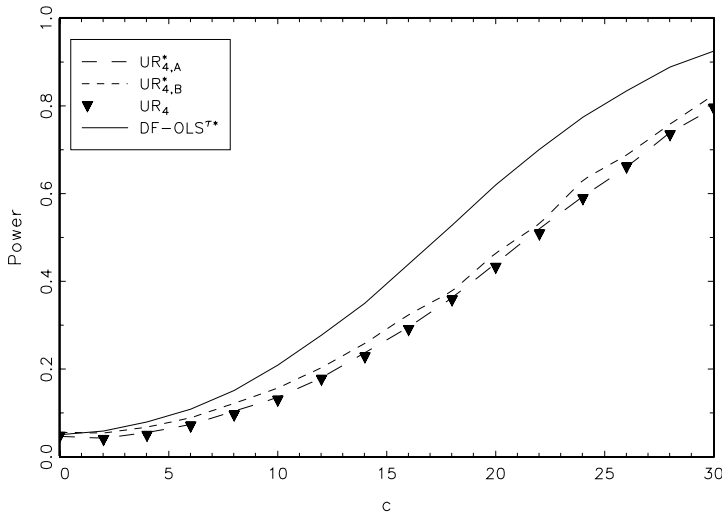

(b) $\alpha=-1$

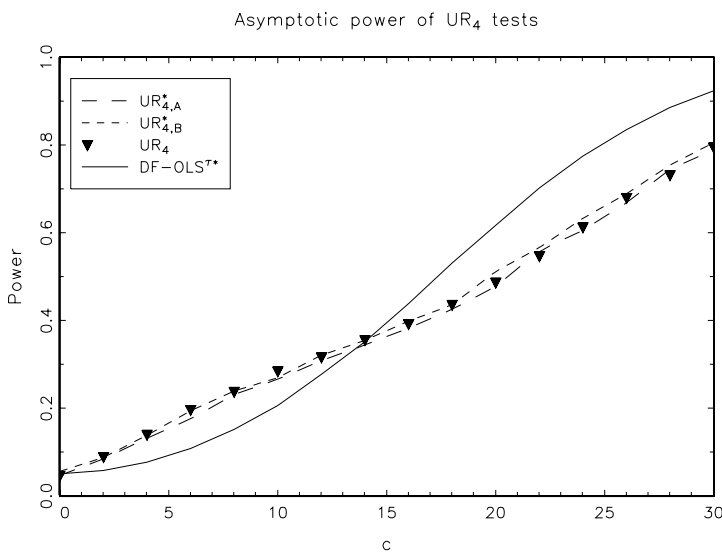

(d) $\alpha=1$

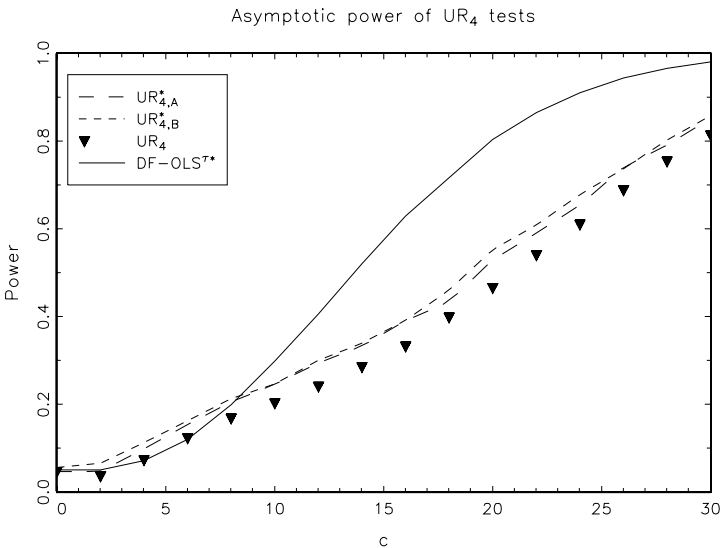

(f) $\alpha=4$

Figure 13: Asymptotic power for $\kappa=0.5$; Single break: $\sigma_{0} / \sigma_{1}=0.2$ and $\tau=0.9$ 
Asymototic power of UR testo

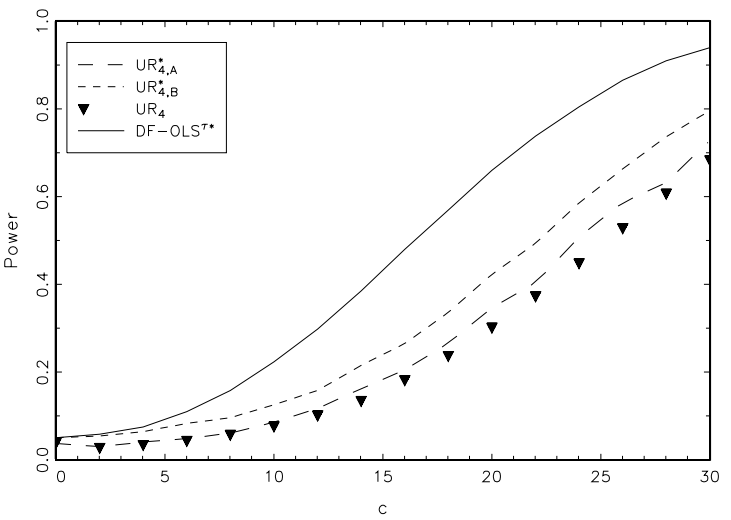

(a) $\alpha=-2$

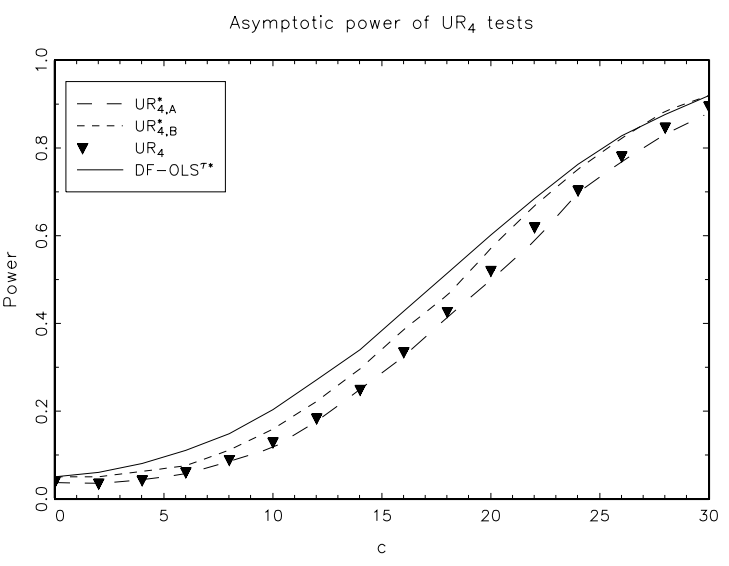

(c) $\alpha=0$

Asymototic power of $\mathrm{UR}_{4}$ tests

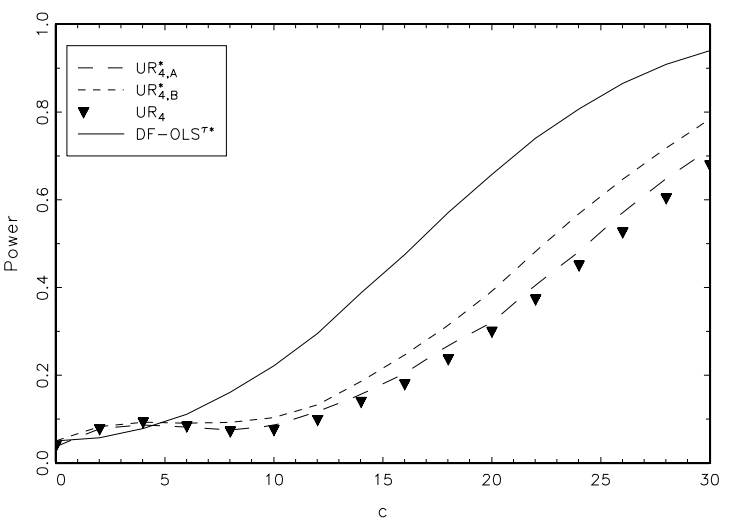

(e) $\alpha=2$
Asymptotic power of UR tests

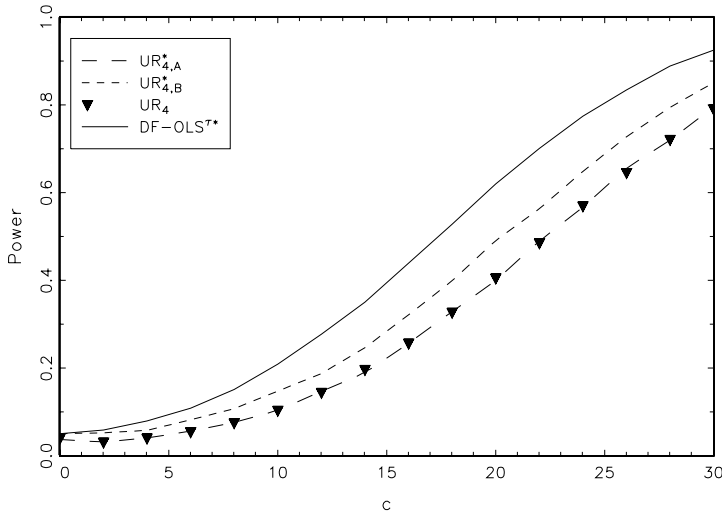

(b) $\alpha=-1$

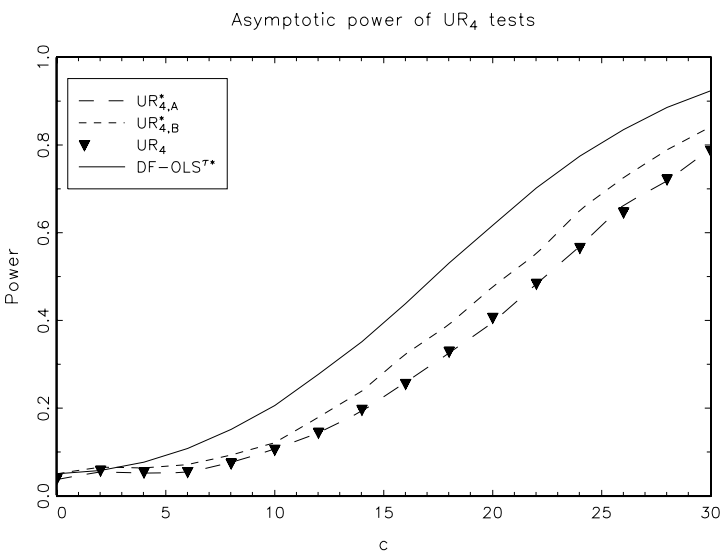

(d) $\alpha=1$

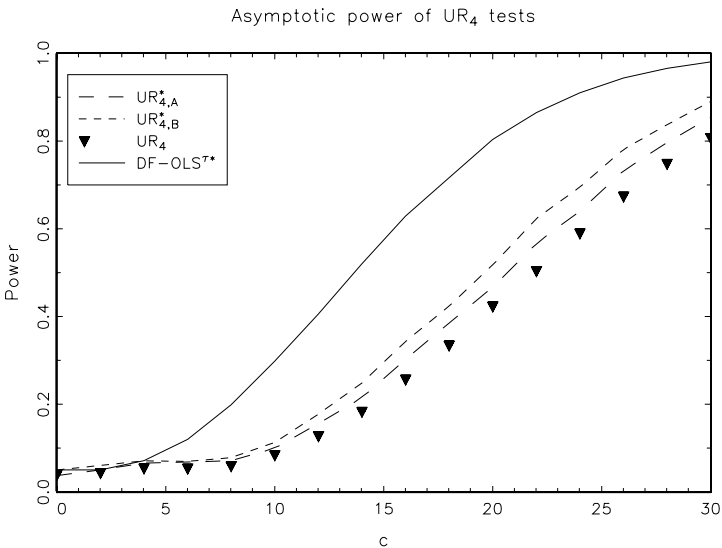

(f) $\alpha=4$

Figure 14: Asymptotic power for $\kappa=1$; Single break: $\sigma_{0} / \sigma_{1}=0.2$ and $\tau=0.9$ 
Asymptotic power of UR tests

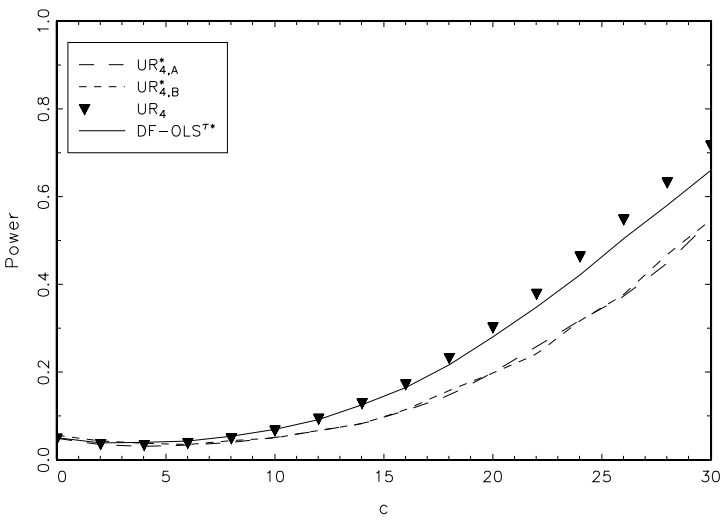

(a) $\alpha=-2$

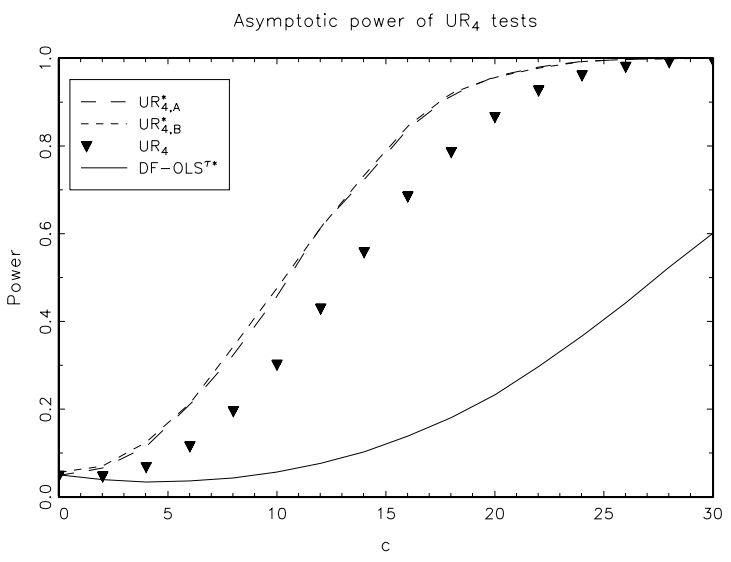

(c) $\alpha=0$

Asymototic power of $\mathrm{UR}_{4}$ tests

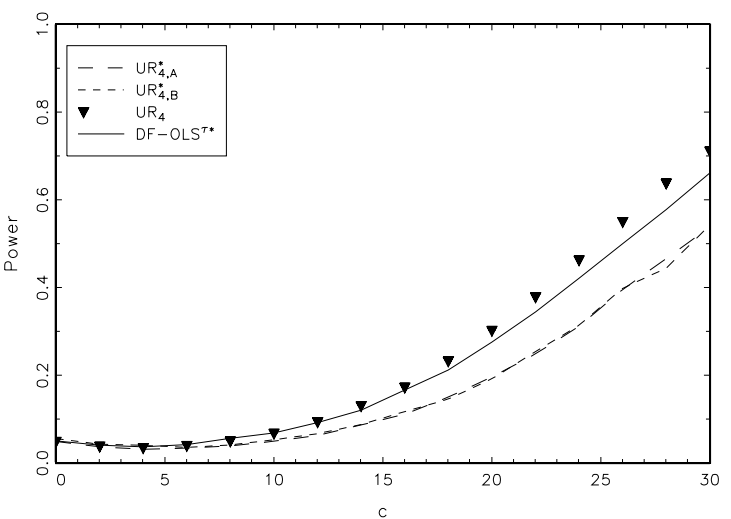

(e) $\alpha=2$
Asymptotic power of UR, tests

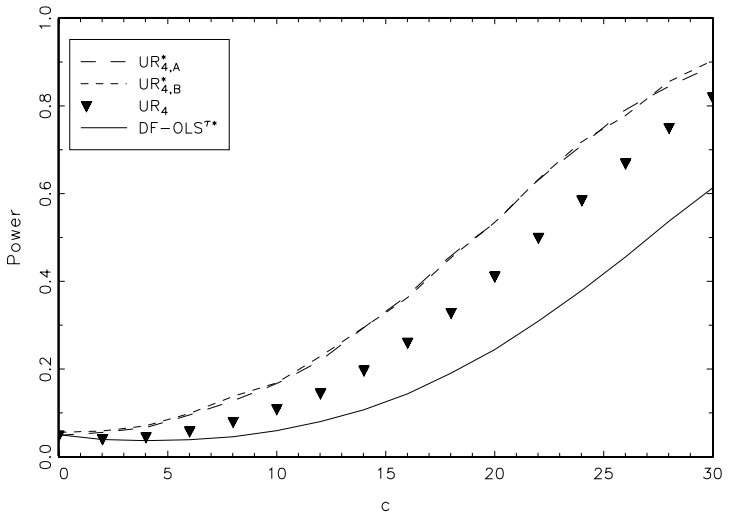

(b) $\alpha=-1$

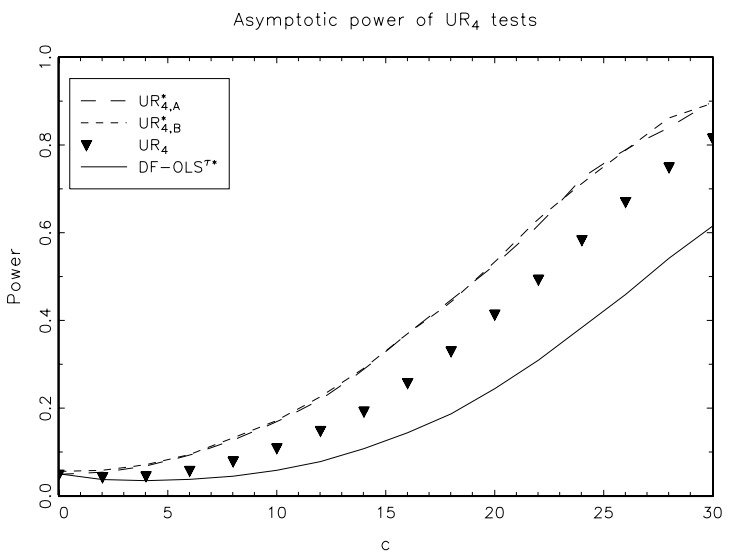

(d) $\alpha=1$

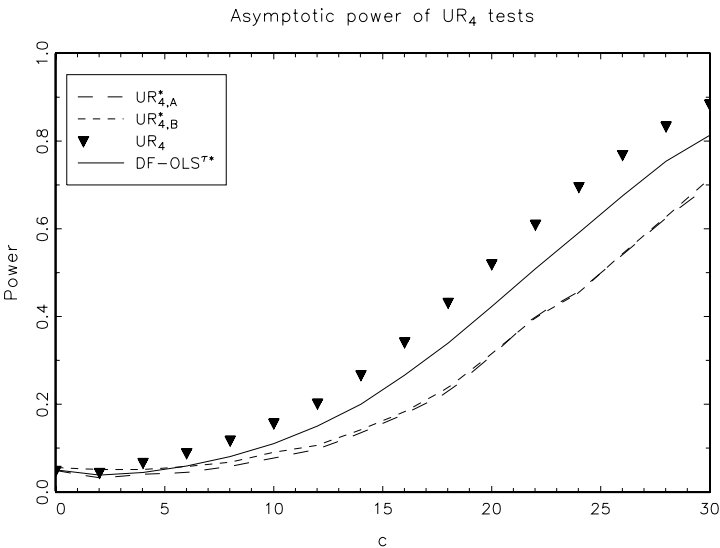

(f) $\alpha=4$

Figure 15: Asymptotic power for $\kappa=0$; Double break: $\sigma_{0} / \sigma_{1}=5$ and $\tau=0.05$ 
Asymototic power of UR tests

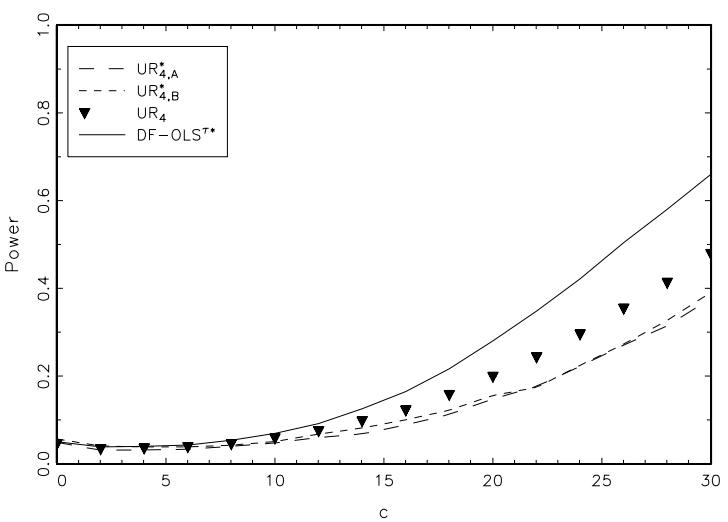

(a) $\alpha=-2$

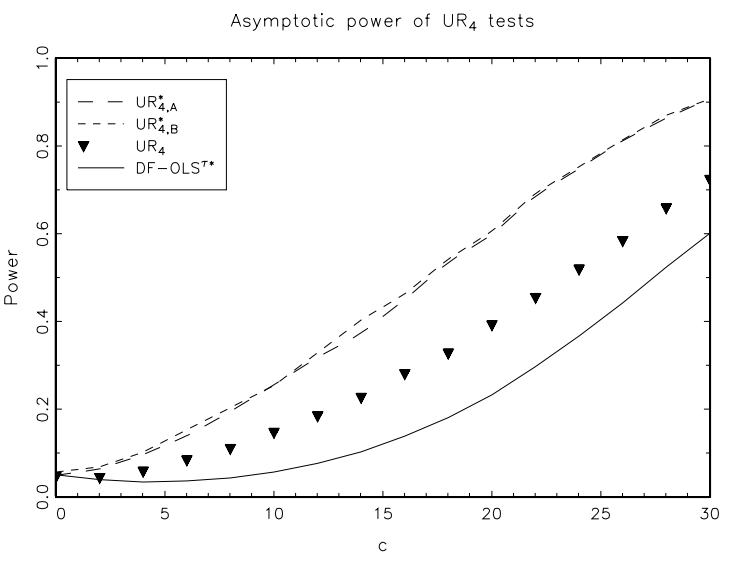

(c) $\alpha=0$

Asymototic power of $\mathrm{UR}_{4}$ tests

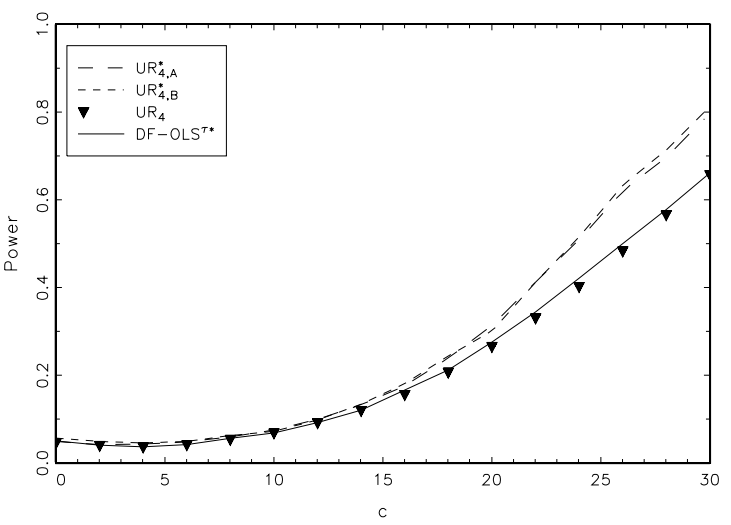

(e) $\alpha=2$
Asymptotic power of $u R_{\text {a tests }}$

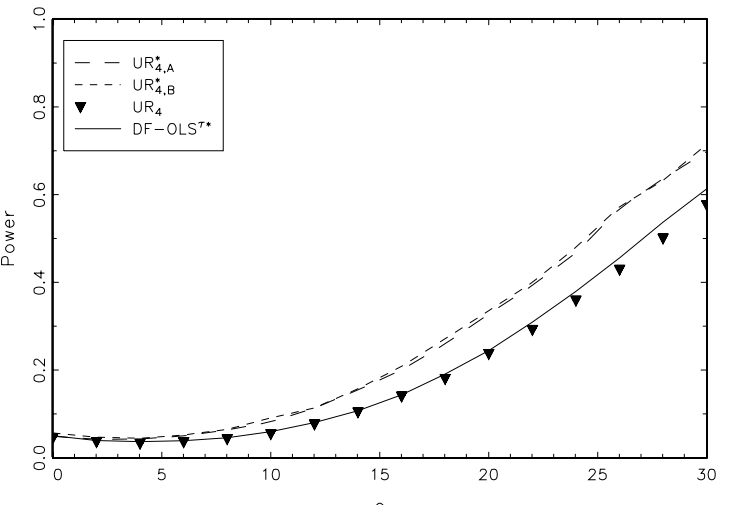

(b) $\alpha=-1$

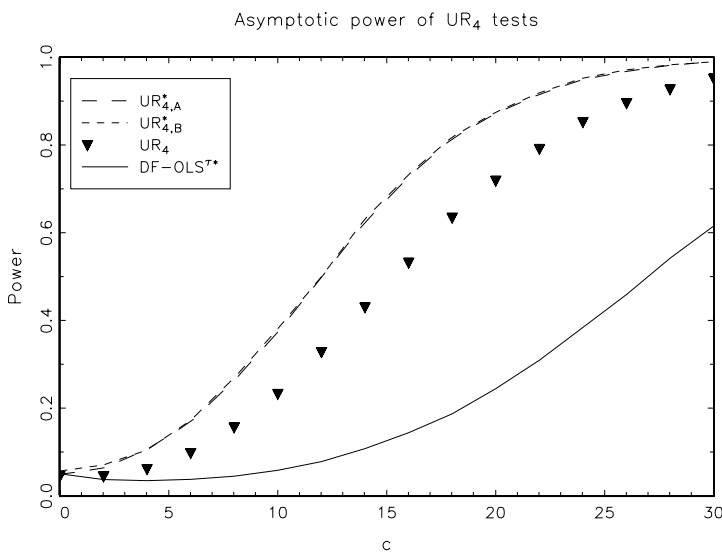

(d) $\alpha=1$

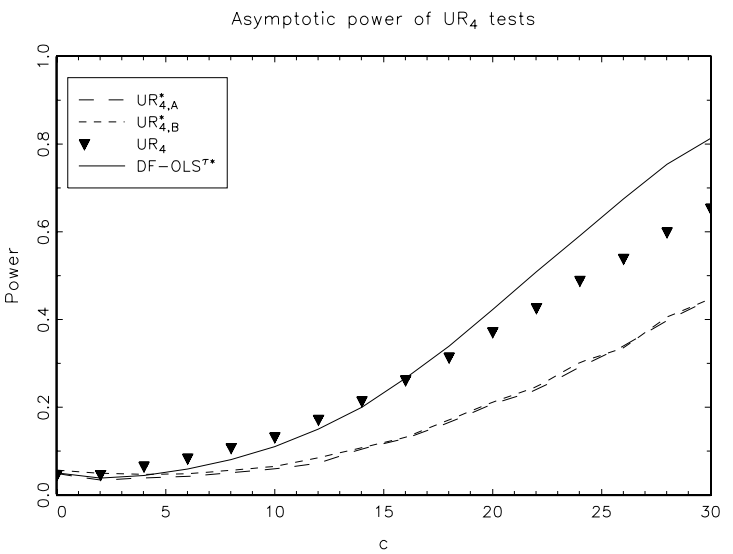

(f) $\alpha=4$

Figure 16: Asymptotic power for $\kappa=0.25$; Double break: $\sigma_{0} / \sigma_{1}=5$ and $\tau=0.05$ 
Asymototic power of UR tests

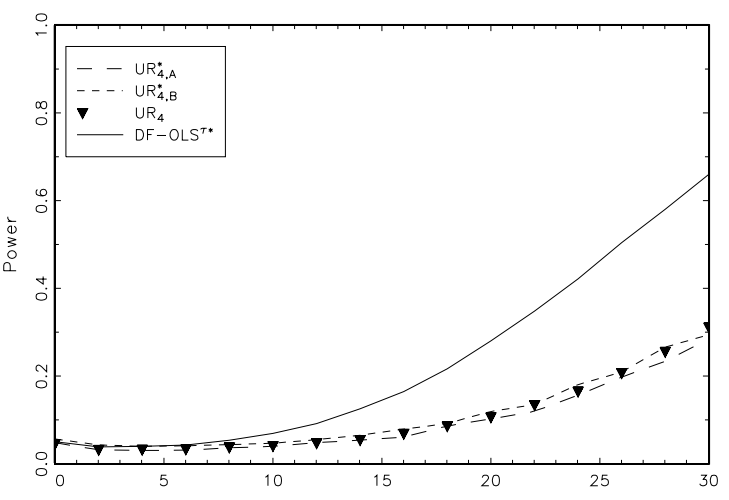

(a) $\alpha=-2$

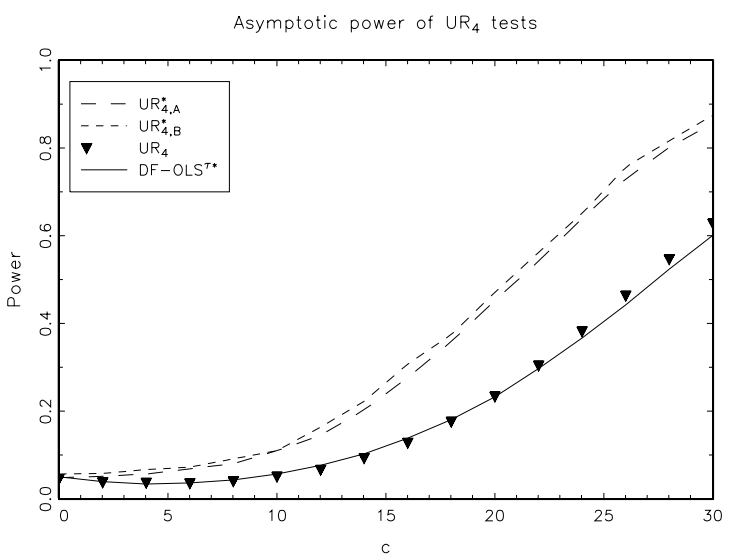

(c) $\alpha=0$

Asymototic power of $\mathrm{UR}_{4}$ tests

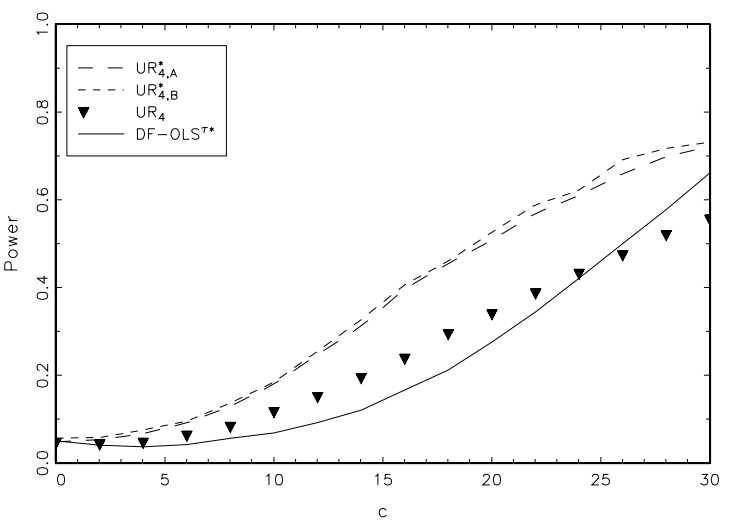

(e) $\alpha=2$
Asymptotic power of $u R_{\text {a tests }}$

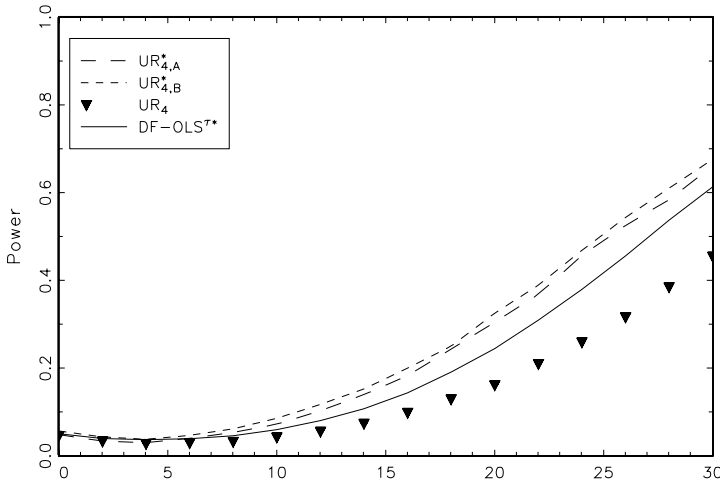

(b) $\alpha=-1$

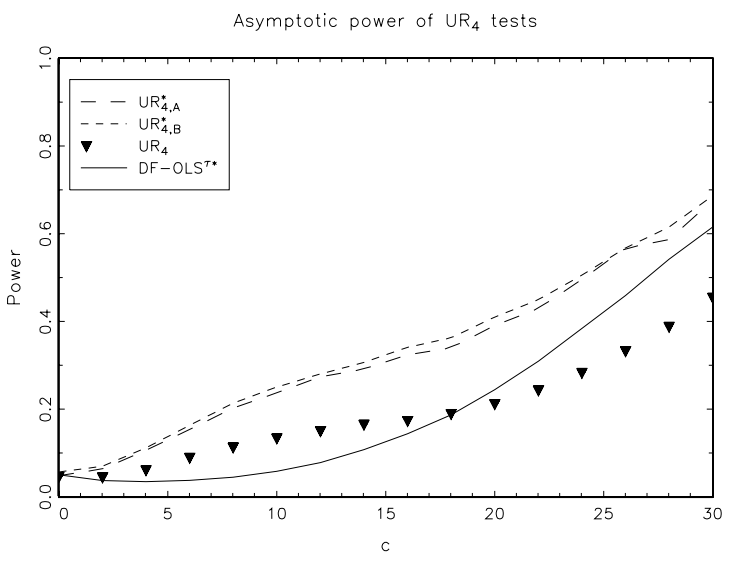

(d) $\alpha=1$

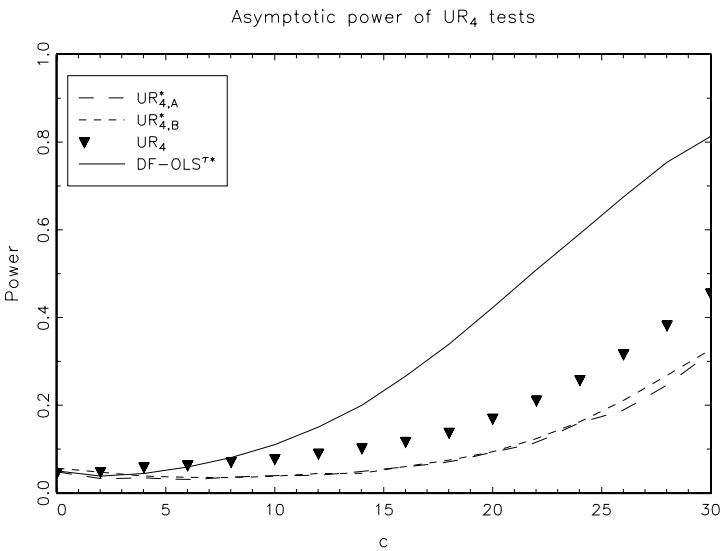

(f) $\alpha=4$

Figure 17: Asymptotic power for $\kappa=0.5$; Double break: $\sigma_{0} / \sigma_{1}=5$ and $\tau=0.05$ 
Asymototic power of UR tests

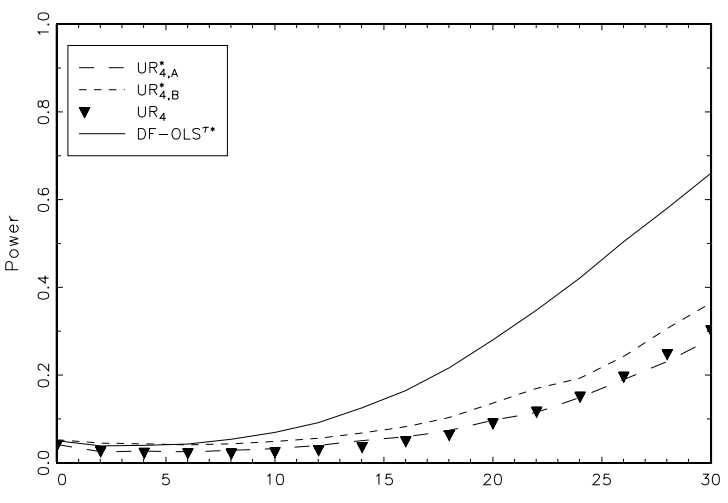

(a) $\alpha=-2$

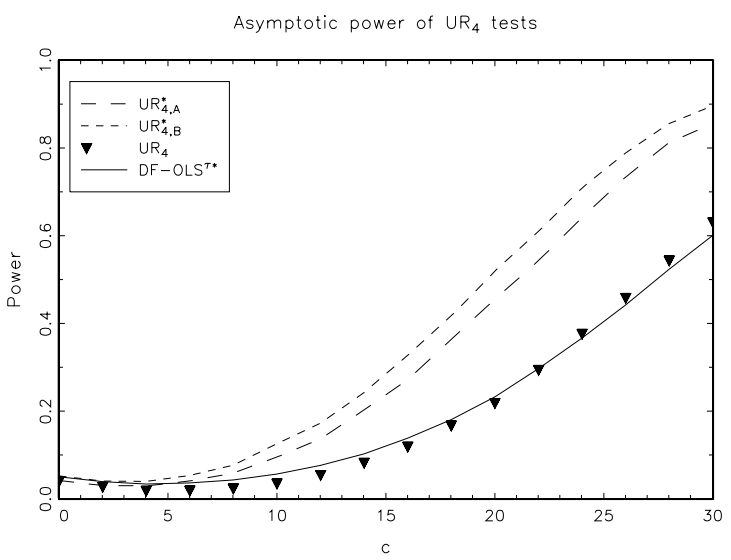

(c) $\alpha=0$

Asymototic power of $\mathrm{UR}_{4}$ tests

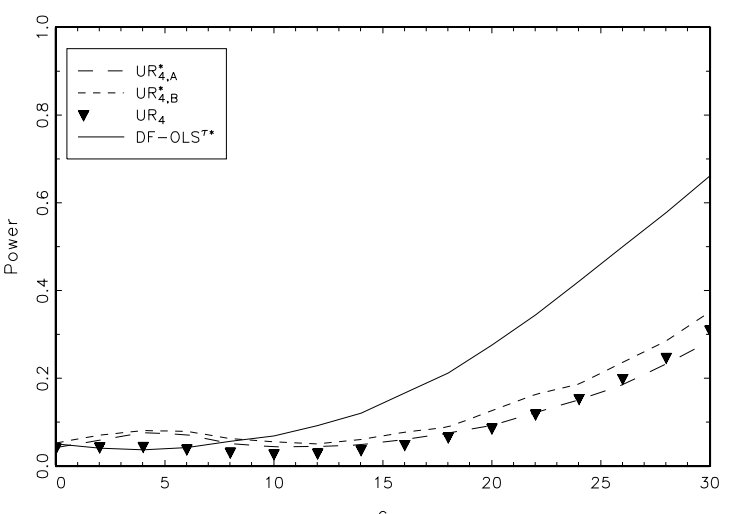

(e) $\alpha=2$
Asymptotic power of $u R_{\text {a tests }}$

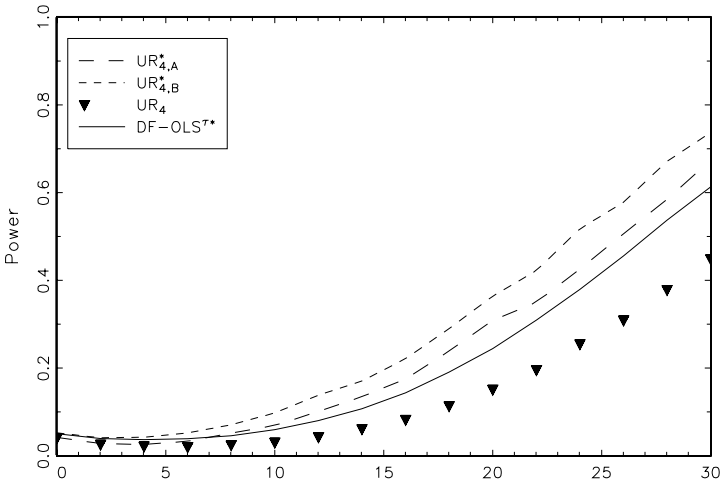

(b) $\alpha=-1$

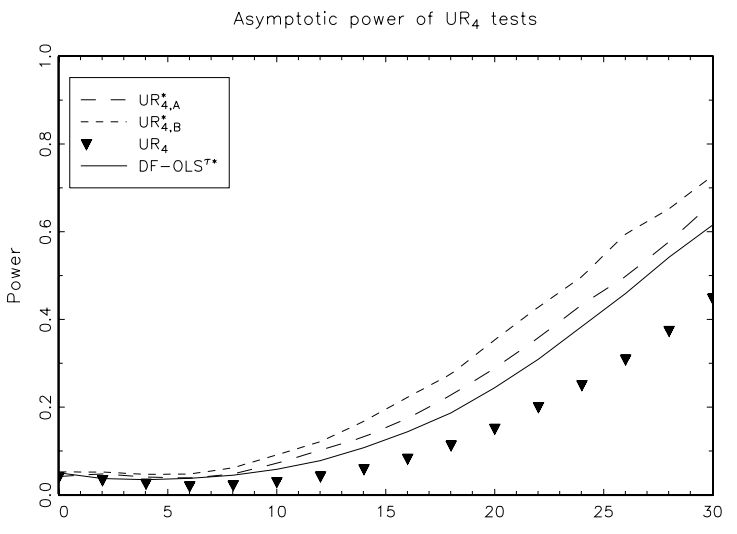

(d) $\alpha=1$

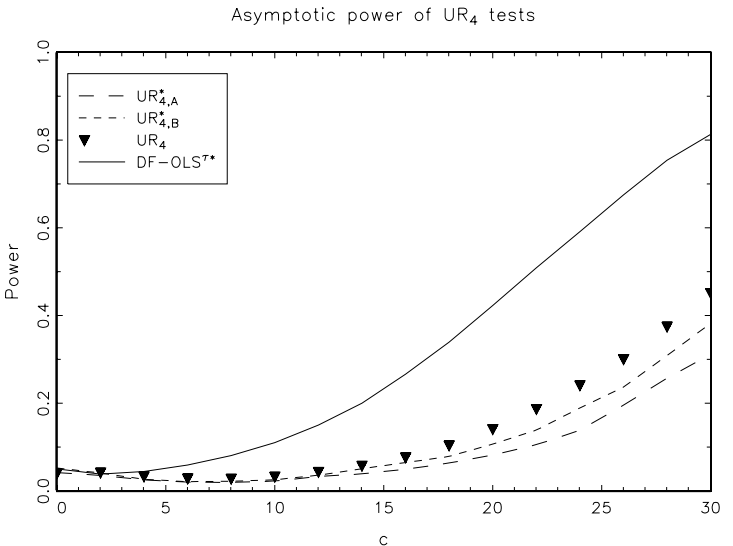

(f) $\alpha=4$

Figure 18: Asymptotic power for $\kappa=1$; Double break: $\sigma_{0} / \sigma_{1}=5$ and $\tau=0.05$ 
Asymototic power of UR tests

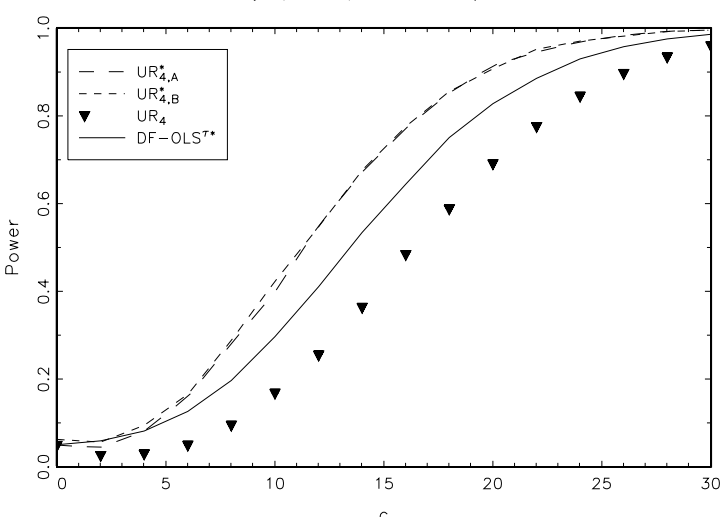

(a) $\alpha=-2$

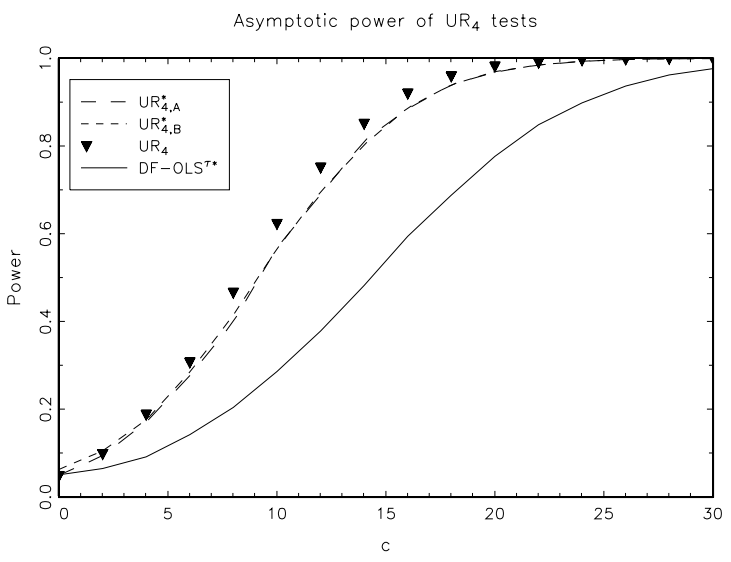

(c) $\alpha=0$

Asymototic power of $\mathrm{UR}_{4}$ tests

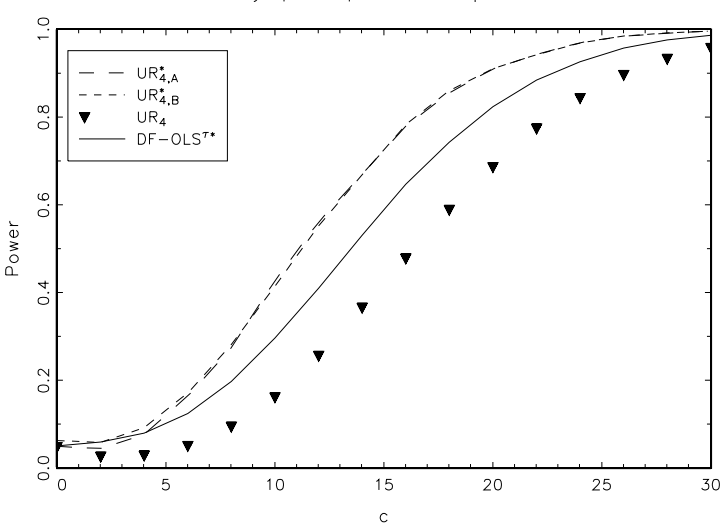

(e) $\alpha=2$
Asymptotic power of UR tests

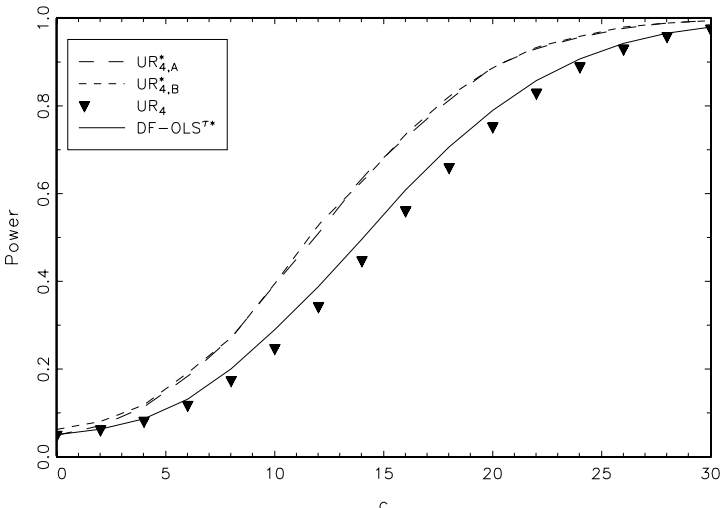

(b) $\alpha=-1$

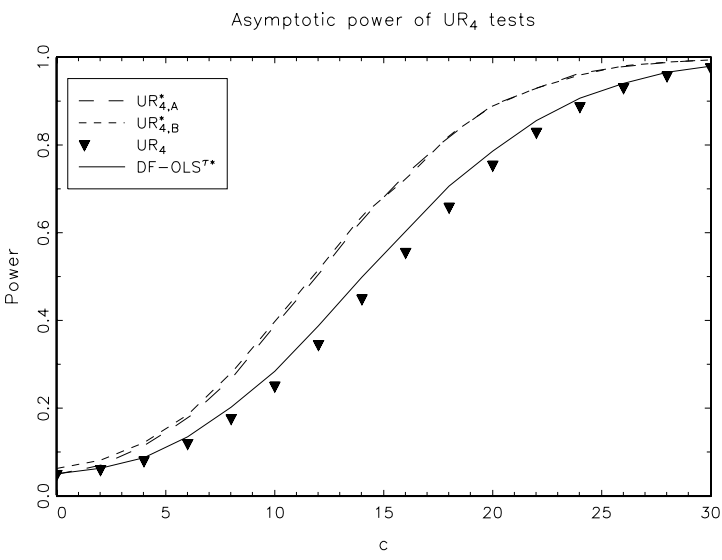

(d) $\alpha=1$

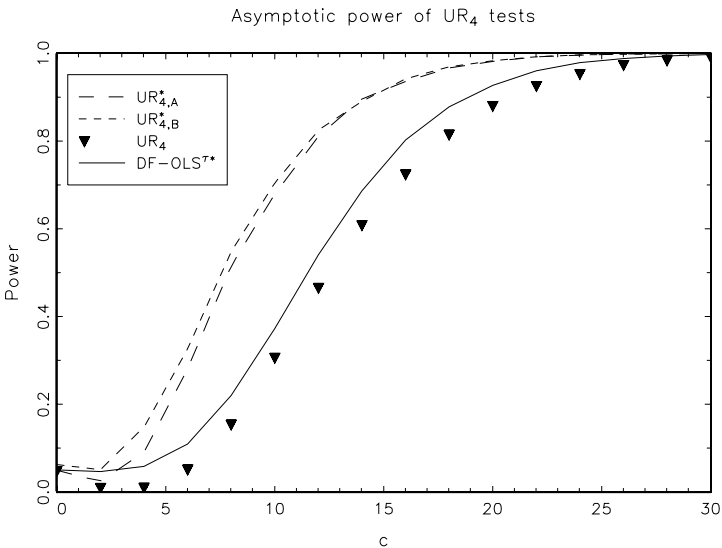

(f) $\alpha=4$

Figure 19: Asymptotic power for $\kappa=0$; Trending: $\sigma_{0} / \sigma_{1}=0.2$ 
Asymototic power of $U R_{4}$ tests

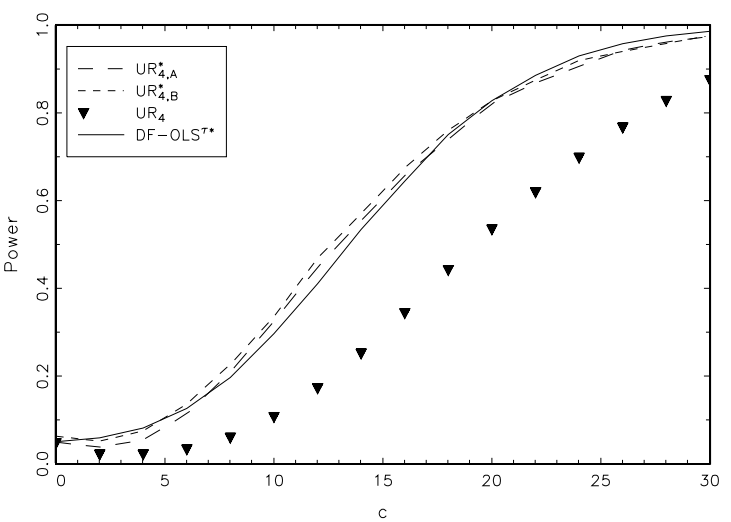

(a) $\alpha=-2$

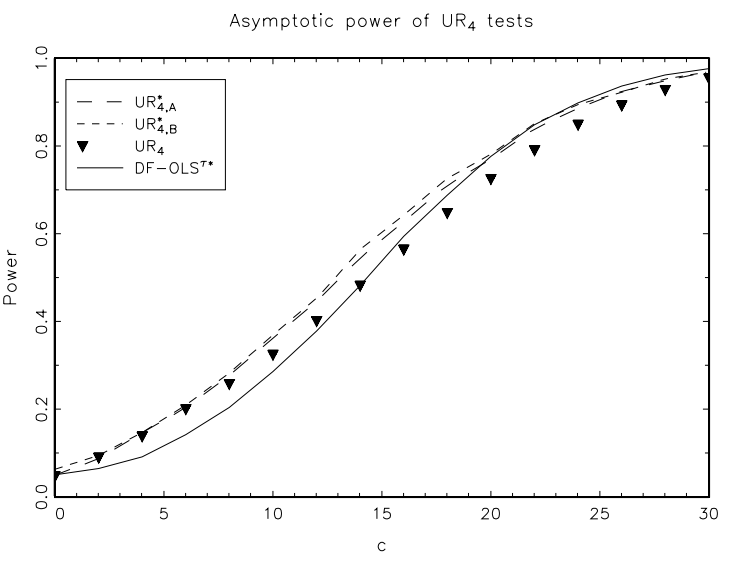

(c) $\alpha=0$

Asymptotic power of $\mathrm{UR}_{4}$ tests

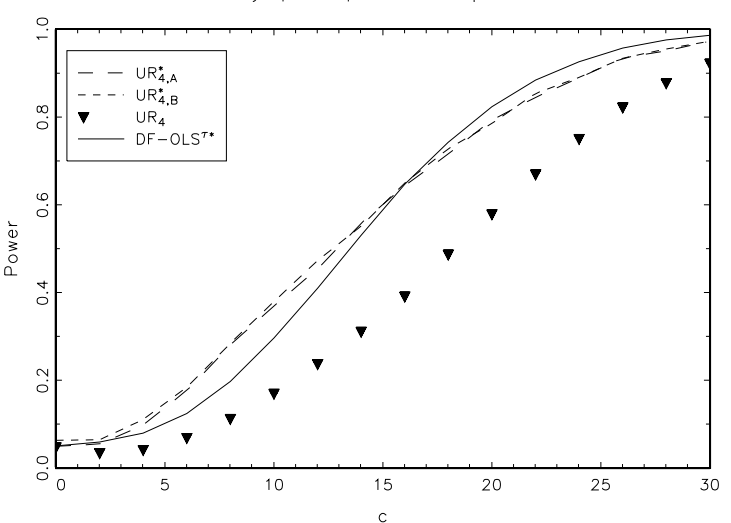

(e) $\alpha=2$
Asymptotic power of $u R_{a}$ tests

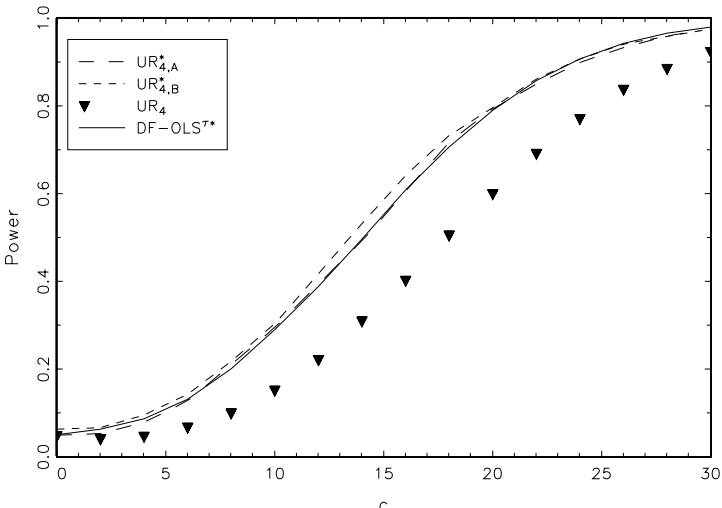

(b) $\alpha=-1$

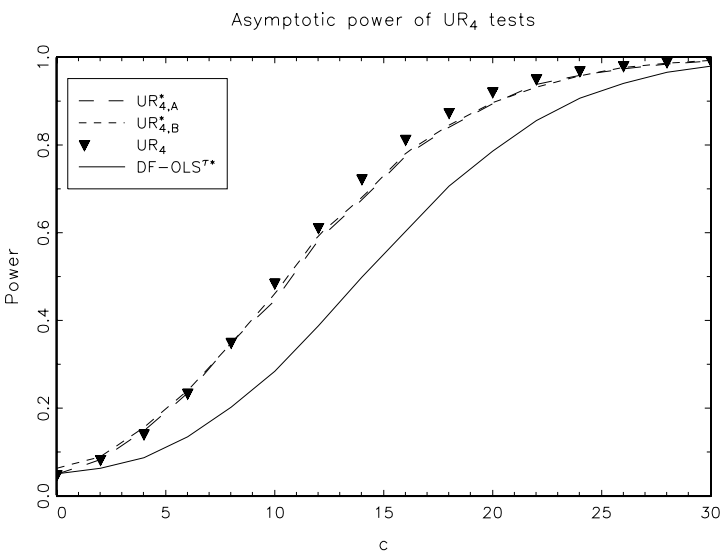

(d) $\alpha=1$

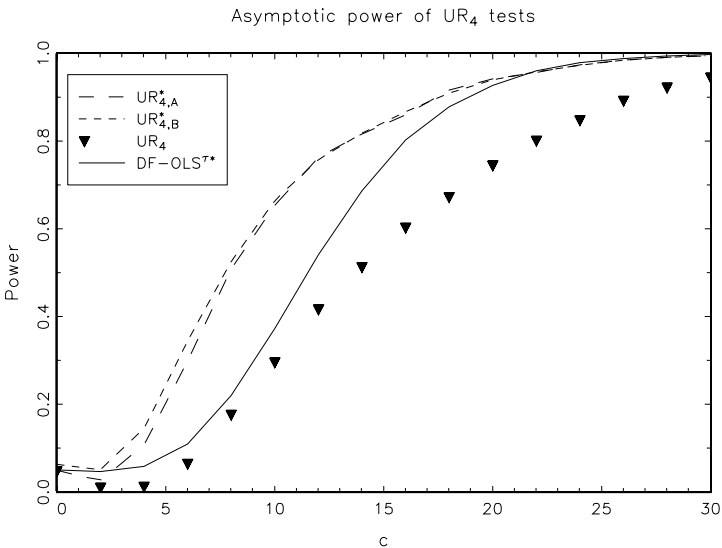

(f) $\alpha=4$

Figure 20: Asymptotic power for $\kappa=0.25$; Trending: $\sigma_{0} / \sigma_{1}=0.2$ 
Acymototic power of UR

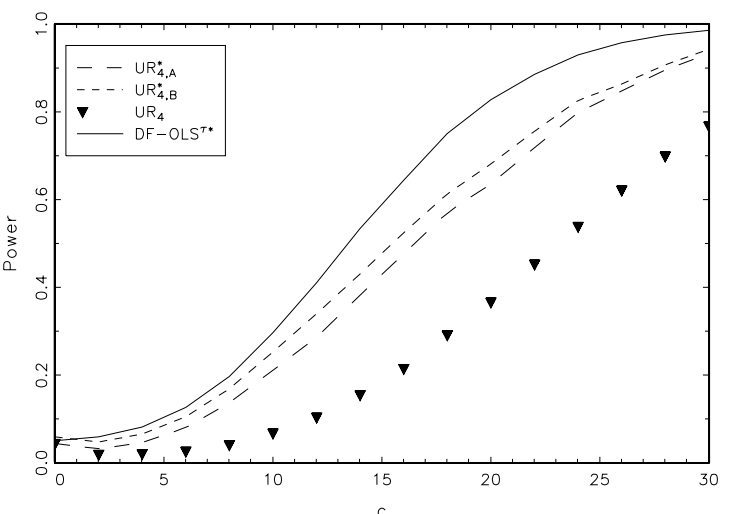

(a) $\alpha=-2$

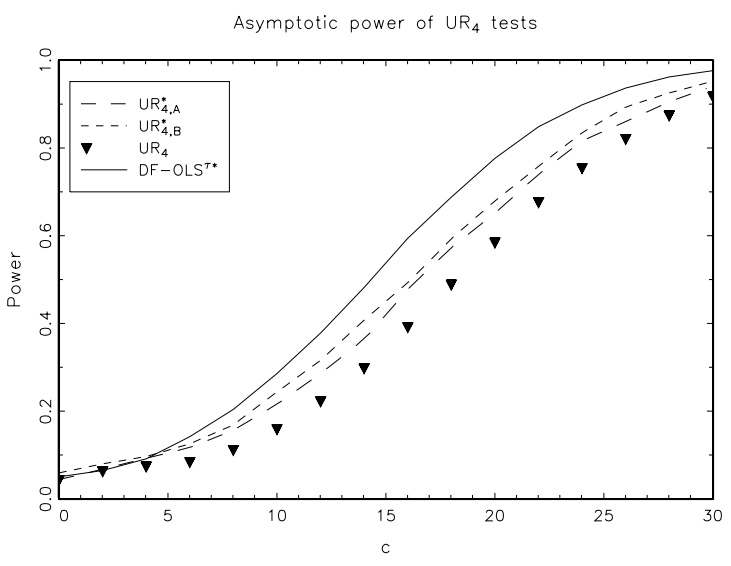

(c) $\alpha=0$

Asymototic power of $\mathrm{UR}_{4}$ tests

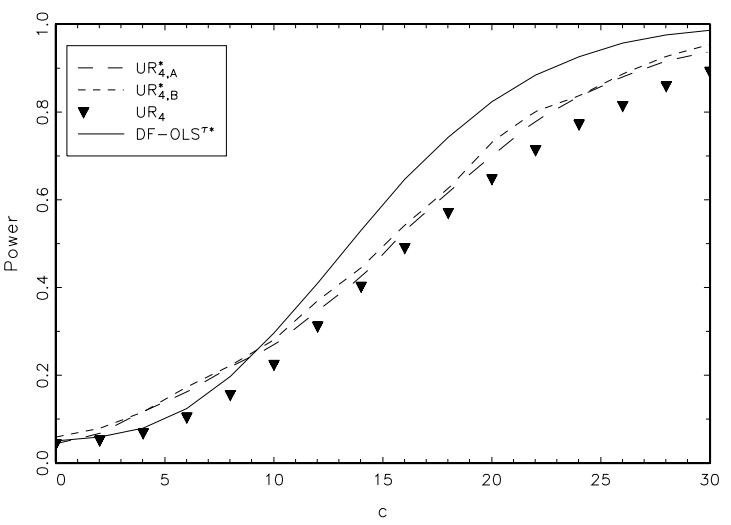

(e) $\alpha=2$
Asymptotic power of UR, tests

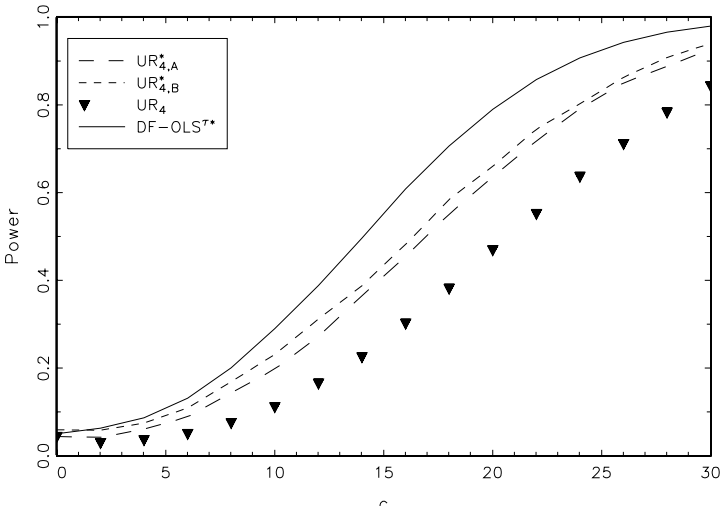

(b) $\alpha=-1$

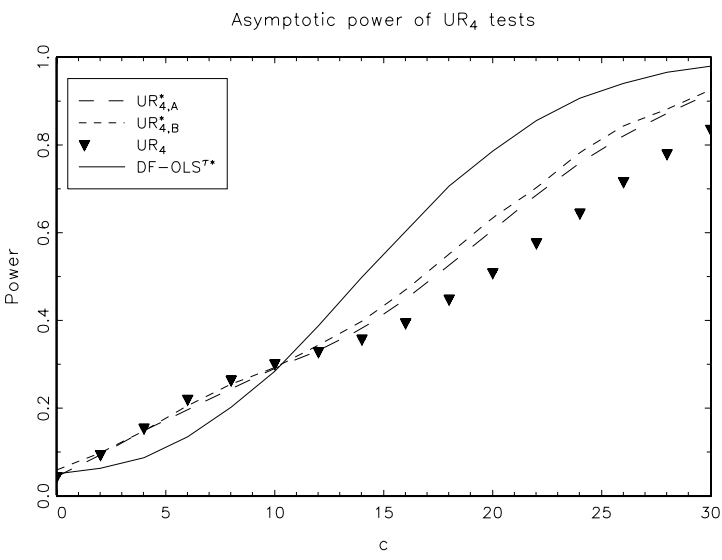

(d) $\alpha=1$

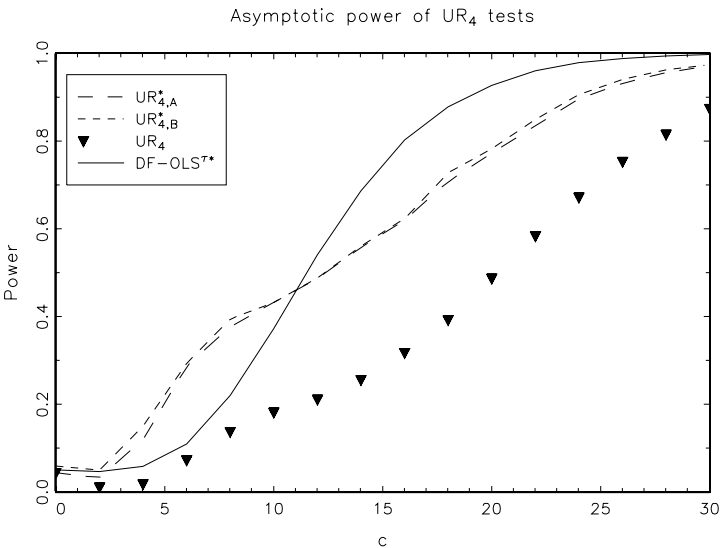

(f) $\alpha=4$

Figure 21: Asymptotic power for $\kappa=0.5$; Trending: $\sigma_{0} / \sigma_{1}=0.2$ 
Asymototic power of UR tests

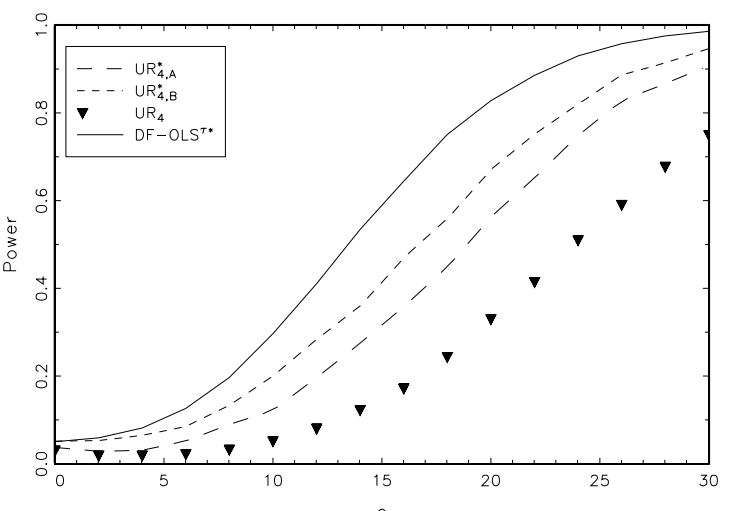

(a) $\alpha=-2$

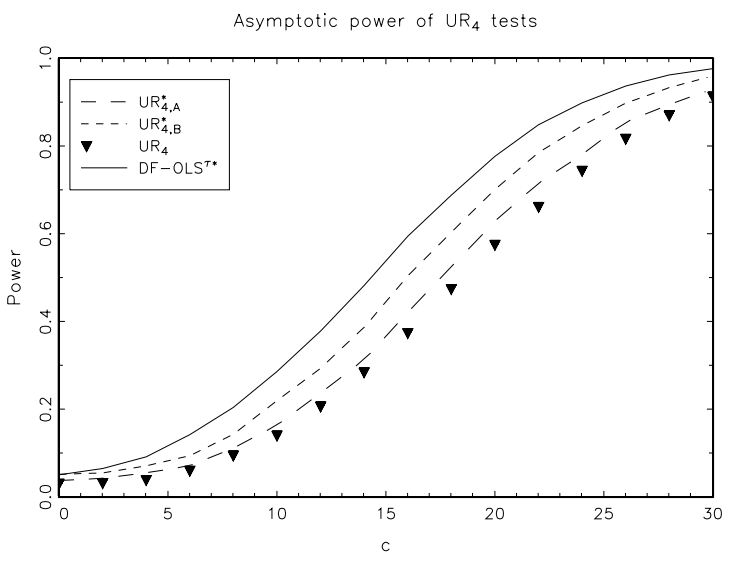

(c) $\alpha=0$

Asymototic power of $\mathrm{UR}_{4}$ tests

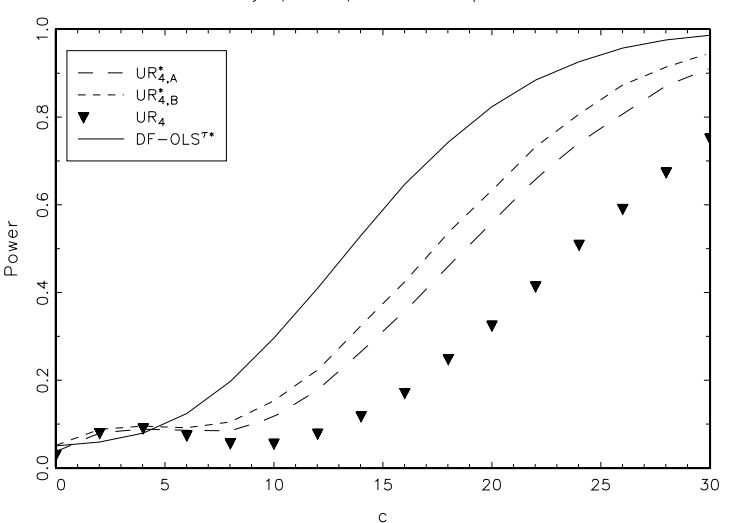

(e) $\alpha=2$
Asymptotic power of UR tests

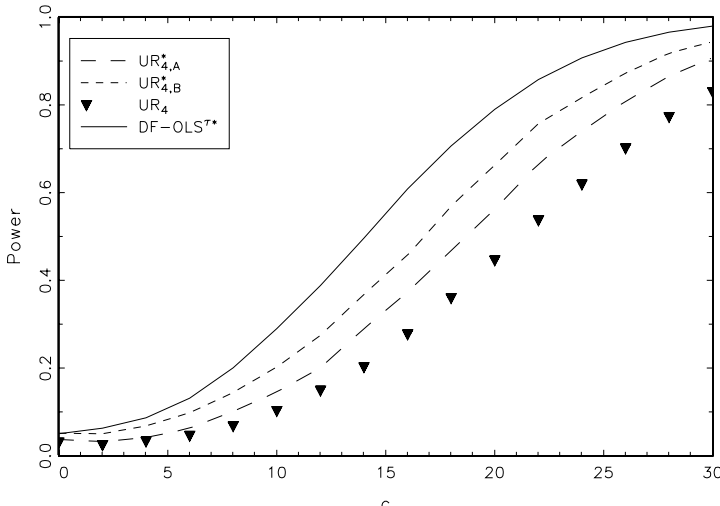

(b) $\alpha=-1$

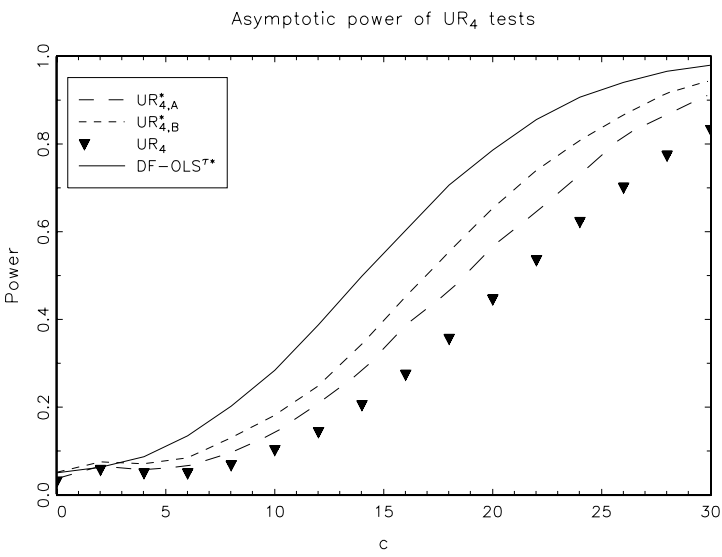

(d) $\alpha=1$

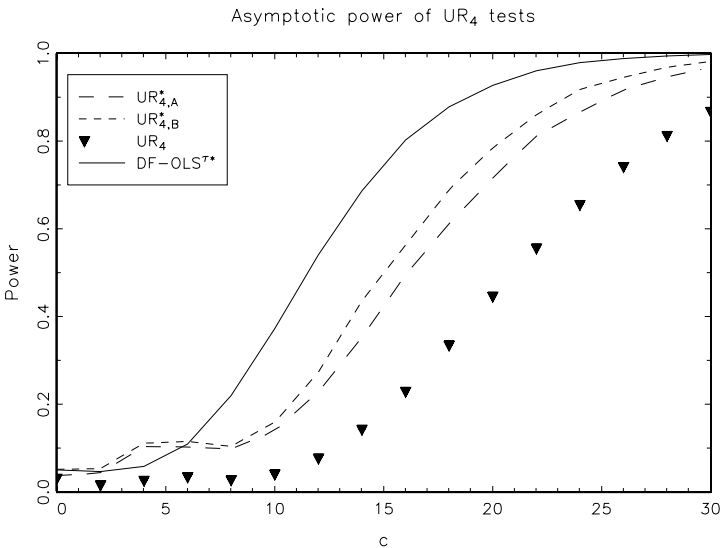

(f) $\alpha=4$

Figure 22: Asymptotic power for $\kappa=1$; Trending: $\sigma_{0} / \sigma_{1}=0.2$ 